\title{
THE ROLE OF TREMATODE PARASITES AS A NUTRITIONAL FOOD SOURCE FOR HIGHER TROPHIC LEVEL CONSUMERS
}

by

\author{
Keira M. McKee \\ Bachelor of Science, 2015, Queen's University, Kingston, ON
}

\author{
A thesis \\ presented to Ryerson University \\ in partial fulfillment of the \\ requirements for the degree of Master of Science \\ in the program of \\ Molecular Science \\ Toronto, Ontario, Canada, 2018 \\ (C) Keira M. McKee, 2018
}




\begin{abstract}
AUTHOR'S DECLARATION
I hereby declare that I am the sole author of this thesis. This is a true copy of the thesis, including any required final revisions, as accepted by my examiners.

I authorize Ryerson University to lend this thesis to other institutions or individuals for the purpose of scholarly research.

I further authorize Ryerson University to reproduce this thesis by photocopying or by other means, in total or in part, at the request of other institutions or individuals for the purpose of scholarly research.
\end{abstract}

I understand that my thesis may be made electronically available to the public. 


\begin{abstract}
The Role of Trematode Parasites as a Nutritional Food Source for Higher Trophic Level Consumers

Master of Science

2018

Keira M. McKee

Molecular Science
\end{abstract}

Recently, there has been a call for the inclusion of free-living stages of trematode parasites in food web models as they are present in high numbers in aquatic ecosystems and serve as prey for higher trophic levels. I investigated the presence of lipids in cercariae, specifically neutral energy reserve lipid stores and long-chain polyunsaturated fatty acids (LC-PUFA), as they are considered the primary energy currency in aquatic ecosystems. Cercariae of all investigated taxa contained distinct neutral lipid compositions and Ribeiroia ondatrae cercariae had significant quantities of total lipids, as well as essential fatty acids (EFA). I also investigated the viability of cercariae as prey items for dragonfly larvae (Leucorrhinia intacta) and detected indistinguishable proportions of EFA in larvae subsisting on equivalent quantities of $R$. ondatrae and Daphnia spp. This suggests that cercariae may contribute to nutrient subsidies in aquatic ecosystems, thereby supporting aquatic organism growth and fitness. 


\section{ACKNOWLEDGEMENTS}

There are many people that I would like to thank for their help and support throughout my graduate journey. First and foremost, I would like to thank both of my research supervisors, Drs. Janet Koprivnikar and Michael T. Arts. They have both helped to guide and shape me into the scientist that I am today, and I am forever grateful for the opportunity they provided me. They have spent countless hours providing feedback and support throughout the planning of my project, during committee meetings, and the writing process, and I cannot thank them enough. They provided me with incredible opportunities, equipment and resources that benefitted my learning and success. I would also like to thank my committee member Dr. Lesley Campbell, for providing excellent insight during our committee meetings, and for always being a positive and encouraging figure throughout my degree. As well as Dr. Andrew Laursen, for providing statistical assistance during my research, and being a member of my defence committee.

There are many lab members that I would also like to thank. From the Koprivnikar lab, M. Baldson and B. Shultz, as well as the Arts lab, E. Morris, T. Preet Parmar, Dr. U. Strandberg, Dr. S. Colombo, S. Al-Alul and D. Milotic. Throughout the two years of my master's tenure these wonderful people have all provided me with much appreciated guidance, advice, and support. I also want to thank L. Santos and J. Nguyen for their assistance in my field collections, and for providing countless laughs and great memories.

I would also like to thank Dr. Pieter Johnson and his PhD students, T. McDevitt-Galles and W. Moss, who allowed me to join their field crew for my data collection. Without their involvement and willingness to help, I would not have been able to accomplish all that I have.

Lastly, I want to say thank you to my family for their continuous support, willingness to lend a listening ear when it was most needed and encouraging me to achieve more than I knew that I was able. Without them none of this would be possible, and I am tremendously grateful. 


\section{TABLE OF CONTENTS}

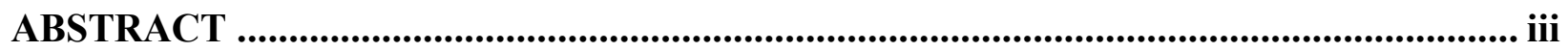

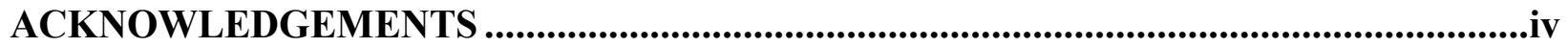

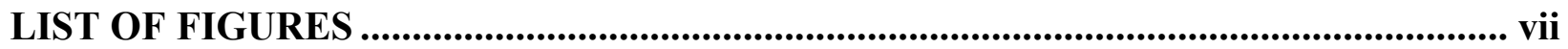

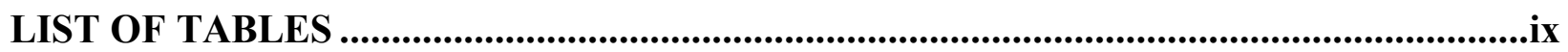

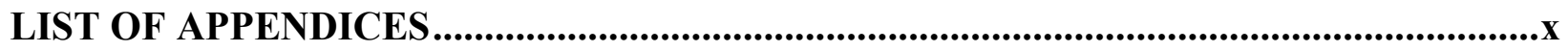

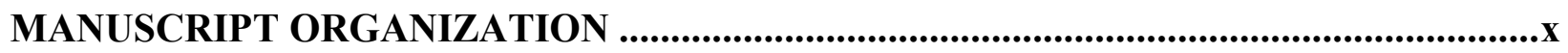

CHAPTER 1: GENERAL INTRODUCTION............................................................................1

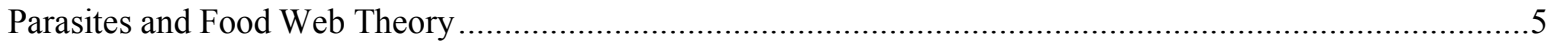

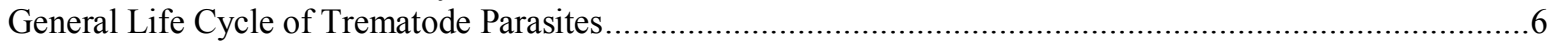

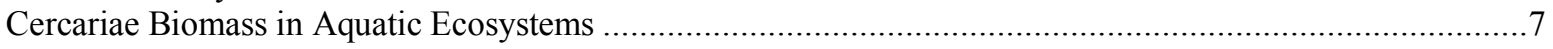

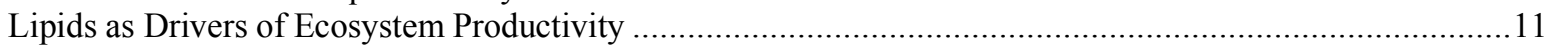

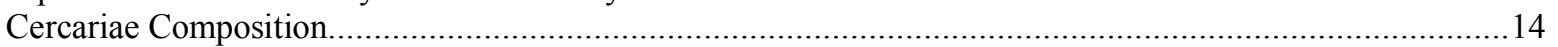

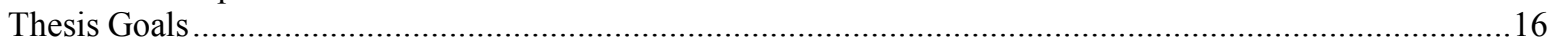

\section{CHAPTER 2: LIPID CONTENT AND FATTY ACID ANALYSIS OF TREMATODE}

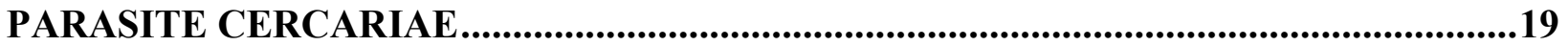

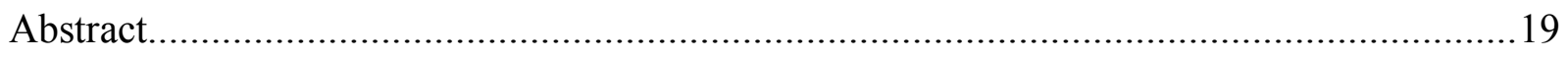

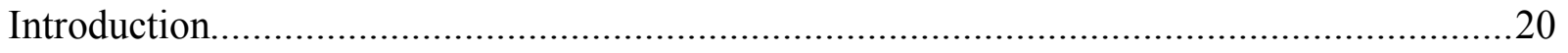

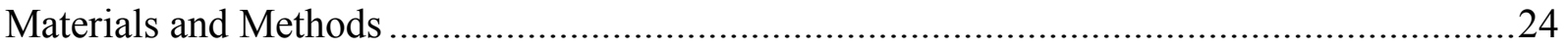

Field Collection and Animal Care ………………………………………………………………….....2

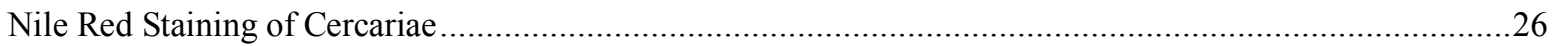

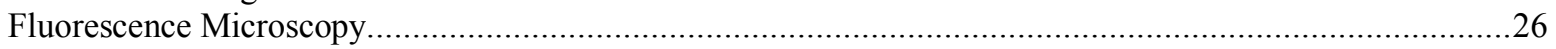

Lipid Fluorescence Binning Technique …………………………………………………………………...2

Neutral Energy Reserve Lipid Concentration Curve ………………………………………………….......2

Fatty Acid Analysis of Ribeiroia ondatrae Cercariae ....…………………………………………………. 31

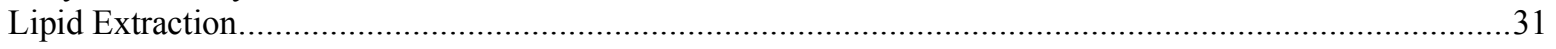

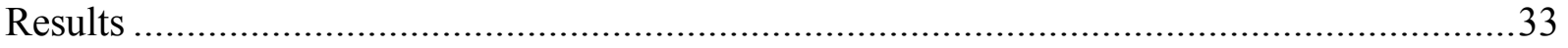

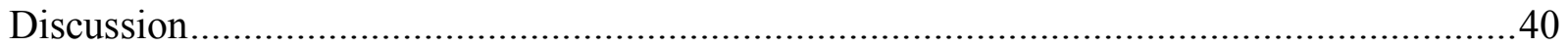

CHAPTER 3: INFLUENCE OF RIBEIROIA ONDATRAE INFECTION ON FATTY ACID PROFILES OF HELISOMA TRIVOLVIS HOST SNAILS......................................52

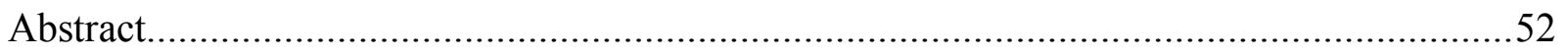

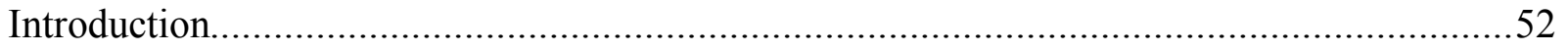

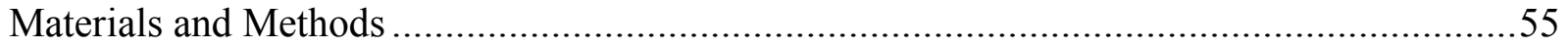

Field Collection and Animal Care ………………………………………………………………....5

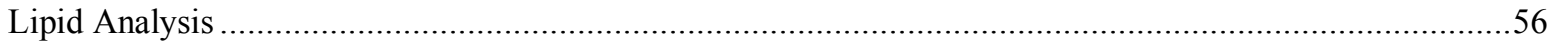

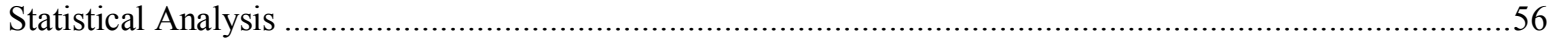

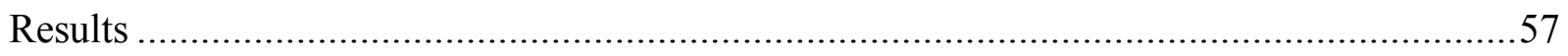

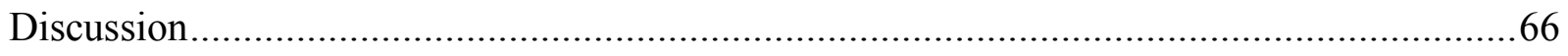




\section{CHAPTER 4: RIBEIROIA ONDATRAE CERCARIAE AS AN ESSENTIAL FATTY ACID-RICH FOOD SOURCE FOR HIGHER TROPHIC LEVEL CONSUMERS ............72}

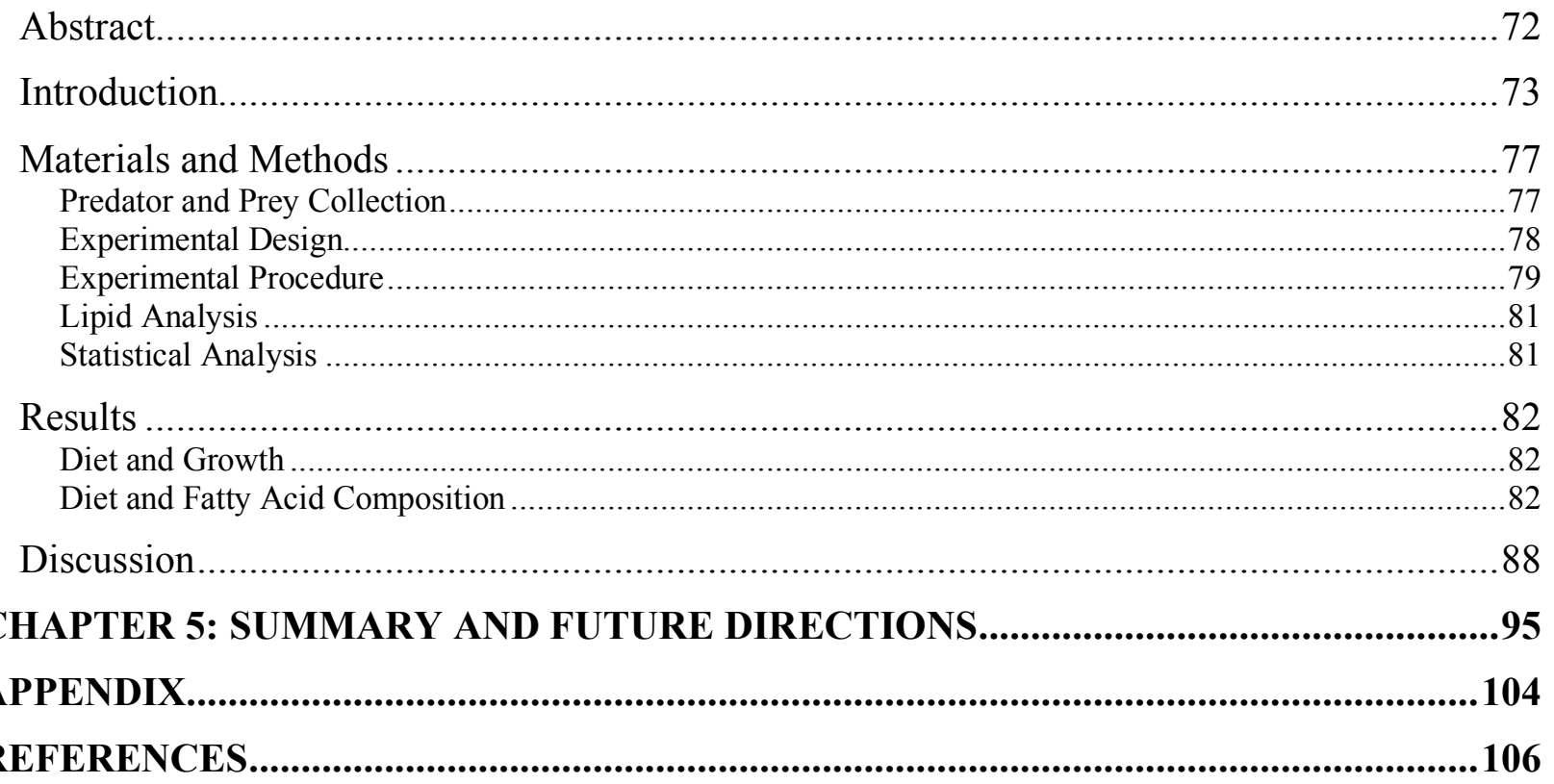




\section{LIST OF FIGURES}

\section{Chapter 1:}

Figure 1.1. Example freshwater food web representing the flow of nutrients from primary consumers to higher trophic levels.

Figure 1.2. Life cycle of the trematode parasites Ribeiroia ondatrae and echinostome-related

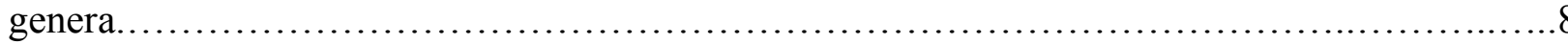

Figure 1.3. Ribeiroia ondatrae cercariae and rediae from Helisoma trivolvis hosts, and Daphnia

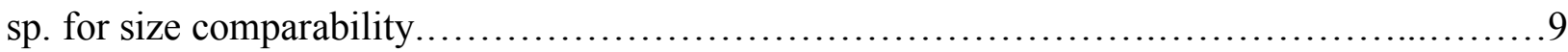

\section{Chapter 2:}

Figure 2.1. Schematic representation of Echinostoma sp. cercariae showing the body regions

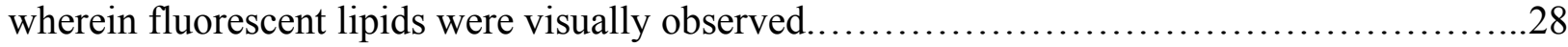
Figure 2.2. Nile red stained fish oil (neutral lipid energy reserve) droplets suspended in ultrasound gel taken with fluorescence microscopy.

Figure 2.3. Concentration curve of Nile red stained fish oil (neutral energy reserve lipid) suspended in ultrasound gel, using fluorescence microscopy........................... 34 Figure 2.4. Nile red stained cercariae of five different species/types.......................36

Figure 2.5. Mean bin assignment of 20 Nile red stained replicates per cercariae-type...........37 Figure 2.6. Estimated mean energy reserve lipids $(\mu \mathrm{g})$ in Nile red-stained cercariae. ...........38

\section{Chapter 3:}

Figure 3.1. Mean total lipid of Ribeiroia ondatrae infected and uninfected Helisoma trivolvis snails grouped by snail infection status

Figure 3.2. Mean proportional and absolute mass fraction of DHA of Ribeiroia ondatrae infected and uninfected Helisoma trivolvis snails grouped by snail infection status...................64 Figure 3.3. Mean proportional SFA of Ribeiroia ondatrae infected and uninfected Helisoma trivolvis snails grouped by snail infection status.

\section{Chapter 4:}

Figure 4.1. A. Leucorrhinia intacta larvae with head width measurement. B. Experimental set-up

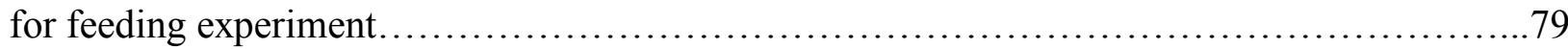


Figure 4.2. Proportion of Cotylurus sp and Posthodiplostomum sp. cercariae eaten by Leucorrhinia intacta dragonfly larvae across range of predator head widths .80

Figure 4.3. Mean \% growth over five weeks of dragonfly (Leucorrhinia intacta) larvae fed one of two different diet treatments (Daphnia spp. or Ribeiroia ondatrae ..........................83 Figure 4.4. Mean proportional SFA, MUFA, and PUFA of dragonfly (Leucorrhinia intacta) larvae fed one of two diet treatments (Daphnia spp. or Ribeiroia ondatrae) Figure 4.5. Mean proportional essential fatty acid (ALA, LNA, EPA, ARA, DHA) of Leucorrhinia intacta fed two diet treatments. .85

Figure 4.6. Mean absolute SFA, MUFA, and PUFA of dragonfly (Leucorrhinia intacta) larvae fed one of two diet treatments (Daphnia spp. or Ribeiroia ondatrae)...........................86 Figure 4.7. Mean absolute essential fatty acid (ALA, LNA, EPA, ARA, DHA) of dragonfly

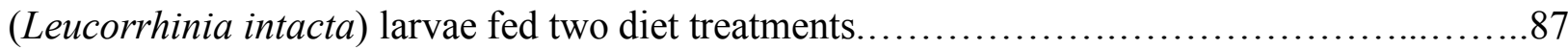




\section{LIST OF TABLES}

\section{Chapter 1:}

Table 1.1. Free sterol and fatty acid composition of cercariae from various trematode parasite taxa.

\section{Chapter 2:}

Table 2.1. Example binning criteria for optical quantification of neutral energy reserve lipids for cercariae of trematode parasites (Echinostoma sp.).

Table 2.2. Fatty acid composition of the Ribeiroia ondatrae cercariae sample in dry weight (DW), wet weight (WW) and proportional (\% of total FA)

Table 2.3. Estimating the annual contribution in dry weight (DW) of EPA and DHA from

Ribeiroia ondatrae cercariae in a California pond.

\section{Chapter 3:}

Table 3.1. Mean proportional fatty acid composition and mass fraction of total lipid in Helisoma trivolvis snails infected and uninfected by Ribeiroia ondatrae............................59 Table 3.2. Mean absolute fatty acid content in Helisoma trivolvis snails infected and uninfected by Ribeiroia ondatrae.

Table 3.3. Univariate factorial ANOVA results of infection and tissue type on the proportional fatty acid and mass fraction of total lipid composition of Helisoma trivolvis snails. Table 3.4. Univariate factorial ANOVA results of infection and tissue type on the absolute mass fraction composition of Helisoma trivolvis snails.

\section{Chapter 4:}

Table 4.1. Individual mass of each prey item (Riberioia ondatrae or Daphnia spp.) and their equivalence in number of individuals. 


\section{LIST OF APPENDICES}

Table A.1. Fatty acid composition of the Ribeiroia ondatrae cercariae sample in dry weight (DW), wet weight (WW) and proportional (\% of total FA).

Table A.2. Mean proportional fatty acid composition of Leucorrhinia intacta larvae fed two diet

treatments.

Table A.3. Mean proportional essential fatty acid composition of Leucorrhinia intacta larvae fed two diet treatments.

Table A.4. Absolute ( $\mu \mathrm{g}$ of FA/mg dry weight) fatty acid composition of Leucorrhinia intacta larvae fed two diet treatments. .130

Table A.5. Absolute ( $\mu \mathrm{g}$ of FA/mg dry weight) essential fatty acid composition of Leucorrhinia intacta larvae fed two diet treatments

\section{MANUSCRIPT ORGANIZATION}

Chapter 2 forms the basis for the investigation of the nutritional composition of trematode parasites using both qualitative and quantitative methods. Chapter 3 investigates the role of trematode parasites in the nutritional composition of their hosts. Chapter 4 investigates the ability for common trematode parasite prey to subsist on a trematode diet, compared to a nutritionally rich Daphnia diet.

For all chapters and sections of the thesis Keira collected all samples and data, ran all experiments, processed the data and ran the statistical analysis and wrote all thesis drafts. Michael Arts and Janet Koprivnikar edited and revised thesis drafts and provided their labs and support for data collection and helped to theorize thesis experiments. 


\section{CHAPTER 1: GENERAL INTRODUCTION}

The concept of food webs is central to the field of ecology and key to our current understanding of the factors that control ecosystem stability, diversity and complexity (Elton 1927; Lindeman 1942; Odum 1953; Hairston and Hairston 1993; Winemiller and Polis 1996; McCann 2000; de Ruiter et al. 2005; Montoya et al. 2006; Pascual and Dunne 2006; Bascompte 2009; Thompson et al. 2012). Charles Elton was the first to conceptualize food webs as a means to organize ecosystem ecology and illustrate that 'food' serves as the connecting link between organisms (Elton 1927). From his work 'Eltonian Pyramids' were conceptualized, which established the pyramidal organization of organisms, where those at the bottom were most abundant, and organisms at the top contained fewer numbers. In 1942, Lindeman combined Elton's perspectives with the energetic work of Lotka (1925) to begin the consideration of biomass within food web models as opposed to number of organisms. Odum (1953) then went on to refine Lindeman's idea of biomass pyramids and suggested that energy (Productivity + Respiration) was the most suitable index for illustrating ecosystem function. Today, the concept of energy as the currency within ecosystem models and food webs remains unchanged.

Food webs represent often varied and complex interactions among species within a community and are created (typically as schematic representations and/or as mathematical models) to characterize feeding relationships (Fig. 1.1) (Elton 1927; Lindeman 1942; Odum 1953; Hairston and Hairston 1993). Generalized parameters are used to characterize and summarize the interactions within food webs; these parameters help us to uncover the general patterns in these complex systems (Hairston and Hairston 1993). They include but are not limited to; species richness (S), number of realized links (L), vulnerability (number of consumer links per resource), generality (number of resource links per consumer), and connectance (LS; proportion of realized links out of the total number of possible links) (Thieltges et al. 2013). Defining an ecosystem in terms of these measures reveals the structure of the community and helps us to quantitatively define the mechanisms that affect its persistence and stability (Paine 1980). Thus, the creation of complete food web models is essential in order to understand the complexity of an ecosystem, as well as to predict the potential consequences of environmental perturbations such as habitat loss, climate change, toxic substances, and/or invasion of exotic species. 
Despite this, food web studies have frequently failed to include one of the most common trophic relationships, i.e. parasitism (Marcogliese and Cone 1997). Parasitism is a two-species symbiotic association in which one organism, the parasite, lives on or in a second species, the host, for a significant period of its life and obtains nutrients from it. Parasitic taxa are traditionally defined to include protozoa, arthropods, and helminthes (Poulin 2011). However, a broader definition exists which also includes all infectious agents of animals including viruses, bacteria and fungi (Van Regenmortel 2003). Within this broader definition, parasites are subdivided into microparasites (bacteria, viruses, fungi and protozoa) and macroparasites (arthropods and helminths). In this thesis, I am solely investigating macroparasites.

Over the last two decades, it has become increasingly apparent that parasites have vital roles in ecosystem processes. Parasites have the ability to influence food web dynamics as they represent a significant portion of Earth's biodiversity (Dobson et al. 2008) and biomass (Kuris et al. 2008) and are similar in abundance to free-living species of comparable size and trophic level (Kuris et al. 2008; Hechinger et al. 2011; Dunne et al. 2013). However, we currently lack knowledge of their biomass on a global scale as their biomass is not resolved from their hosts, and depictions of food webs (whether graphical or mathematical) typically do not specifically identify parasite components due to their multi-host lifecycles (Lafferty et al. 2008; Bar-On et al. 2018). Many macroparasites including cestodes, nematodes, acanthocephalans and flatworms such as trematodes, have complex and multi-host life cycles. The completion of the life cycle relies on the trophic transmission through the use of intermediate and definitive hosts. This feature of their life cycle gives parasites the ability to drastically affect trophic interactions within food webs at numerous junctions and through numerous mechanisms. The most common predator-prey links are present in the form of concomitant predation where a parasitized host is consumed by a predator. The next most common pattern is trophic transmission where a predator consumes an infected host and becomes infected. Finally, there is direct predation on free-living parasite life stages.

A transformative study by Marcogliese and Cone (1997) drew attention to the importance of incorporating trematode parasites into food webs in order to gain a complete understanding of ecosystem function. They suggested that including parasites in food web models is necessary in order to accurately assess trophic interactions, linkage strength and food-web structure dynamics. They also suggested that this would provide an opportunity to reassess the fundamental food web 
properties (Marcogliese and Cone 1997). The need to consider parasites in this context has since been reaffirmed by many others (Elton 1927; Campbell et al. 1980; Goldwasser and Roughgarden 1993; Huxham et al. 1995; Lafferty et al. 2006; Lafferty et al. 2008; Byers 2009; Sukhdeo 2012; Wood et al. 2015). As the literature accumulates on the staggering biomass of parasites in aquatic ecosystems, and the realization that their free-living infectious stages can serve as prey for higher trophic levels, it is imperative that we investigate their potential to serve as a nutrient rich energy source, one which may be contributing substantially to the flux of energy and nutrients in aquatic and terrestrial ecosystems. 


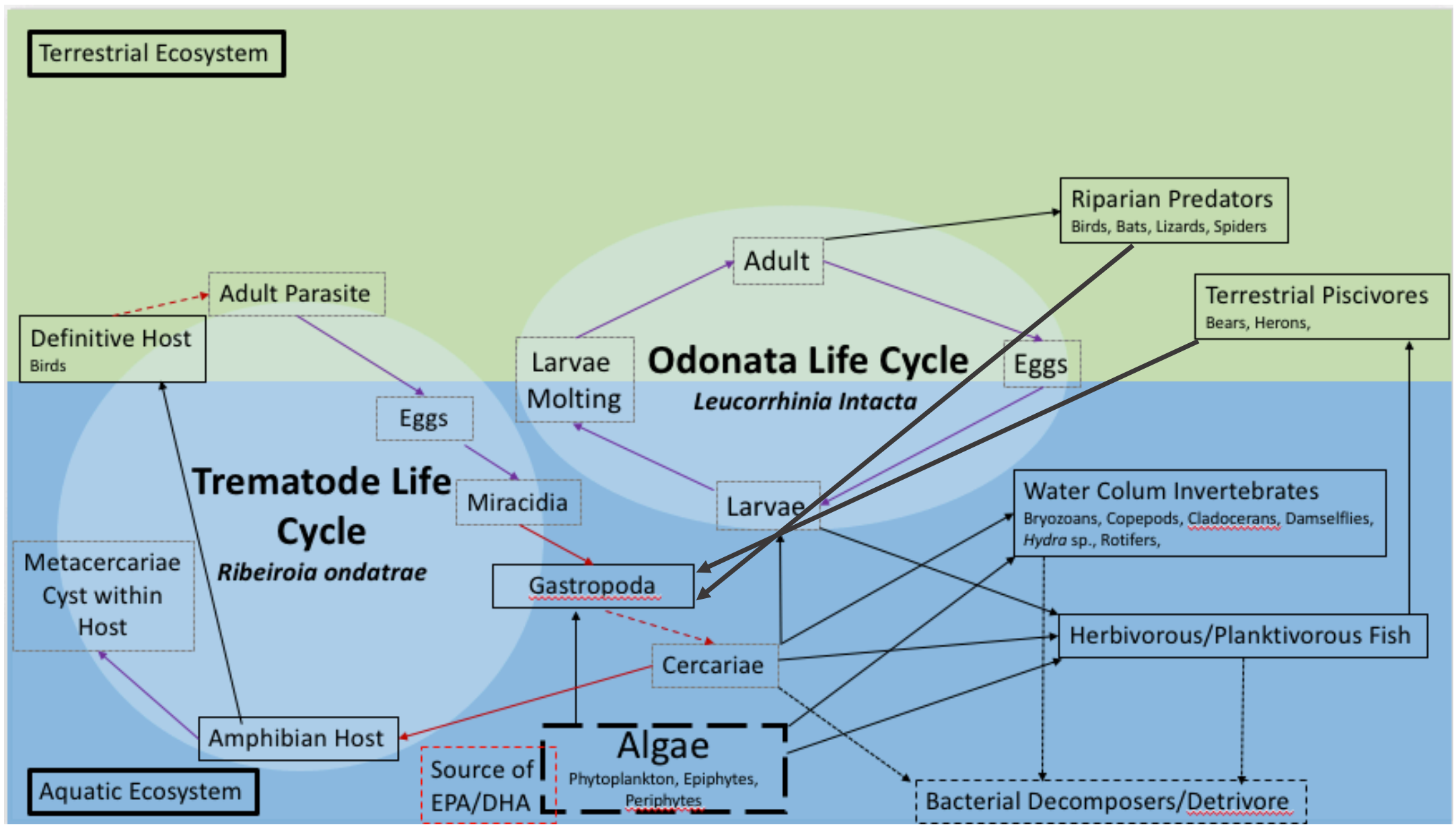

Figure 1.1. Example freshwater food web representing the flow of nutrients from primary producers such as algae to free-living parasite infectious stages (trematode cercariae here) and higher trophic level consumers. Purple lines represent life history stages for both a model trematode (Ribeiroia ondatrae) and secondary consumer (a larval odonate). Black lines represent consumption and nutrient flow. Red lines represent parasite infection, with dashed lines indicating consumption of host tissue and possible nutrient acquisition. Red dashed box indicates high source of EPA/DHA in algae. 


\section{Parasites and Food Web Theory}

Three generally accepted food web models include the cascade model (Cohen et al. 1990), the niche model (Williams and Martinez 2000), and the nested hierarchy model (Cattin et al. 2004). These models focus on the relationship of prey to predator body size, where consumers eat prey that are smaller than themselves (Dunne et al. 2013). Parasites fit poorly into current food web models due to the fact that they rely on energy from larger (host) organisms and because of their complex life cycles (Dunne et al. 2013). Initial efforts identified that adding parasites into typical food-web models altered several key food-web metrics (Marcogliese and Cone 1997; Poulin 1999; Loreau et al. 2005) including, an increase in species richness, number of links, linkage density (number of links per species), food chain length and connectance values (the number of links/total links possible) (Huxham et al. 1995; Thompson et al. 2005; Hernandez and Sukhdeo 2004; Lafferty et al. 2006; Sukhdeo 2012; Dunne et al. 2013). Linkage density and connectance are particularly important as they serve as baseline indicators of ecosystem stability (May 1973; Sukhedo et al. 2012). The ability for parasites to alter essential food web properties illustrates the need for their inclusion in food web models.

Although parasites alter food web structure, there is further evidence that these changes are not unique to their mode of living, (i.e. parasitism) per se and may be more attributable to the effect of adding $20-40 \%$ new species, (i.e. previously unaccounted for) to food web matrices (de Ruiter et al. 2005; Montoya et al. 2006; Raffel et al. 2008; Sukhdeo 2012; Dunne et al. 2013). For example, Dunne et al. (2013) and Lafferty et al. $(2006,2008)$ analyzed the effects of adding parasites to foods webs typical of the Carpinteria Salt Marsh in Santa Barbara County, California; a network structure of seven highly resolved coastal estuary/marine food webs. They found that diversity and complexity increased upon inclusion of parasites into their models (Dunne et al. 2013). Explicitly including parasites in their food web model was found to uniquely alter the structure and function/dynamics of the system because of the role that parasites played in serving as resources for predators of their hosts, having broad feeding niches due to their complex life cycles, their close and extended relationship with their hosts, predator-prey body size and energy transfer (Lafferty et al. 2008; Dunne et al. 2013). They identified 615 parasite-host links and 910 predator-parasite links relative to 505 'conventional', (i.e. non parasitic) predator-prey links. Thus, even though the parasites account for less than $50 \%$ of the links in that system, they concluded that food web models that exclude parasites underestimate a 
large portion of the biodiversity in the system, omit links and miscalculate the true topological structure of the ecosystem (Lafferty et al. 2008). This previously unrecognized, and potentially substantial, flow of energy and nutrients has numerous implications for ecosystem function including; changes to food web topography (explains the structure of the ecosystem), trophic transmission, and overall food web energetics (Johnson et al. 2010).

The creation of new food web models, such as the inverse niche model, aims to properly address the role and impact of parasites in food webs (Warren et al. 2010). The realization that the impact of parasites may be highly significant not only stems from their effects on food web properties, but also from their substantial biomass in aquatic ecosystems (Lafferty et al. 2010). Outside of their involvement in trophic interactions via consumption of infected hosts, the immense number of free-living parasite infectious stages entering into aquatic ecosystems on a daily basis serves as another potential pathway by which they may affect food webs, especially if they represent a significant source of energy and nutrients (Preston et al. 2013). Additionally, the life cycles of numerous macroparasites have free-living infectious stages which could contribute to food web energetics. Given that the vast majority of studies regarding parasites and food webs have involved digenean trematodes (Johnson et al. 2004, Preston and Johnson 2010), these will serve as the focus here.

\section{General Life Cycle of Trematode Parasites}

The life cycle of digenean trematode parasites is completed through the use of multiple hosts: a vertebrate definitive host in which adult worms reside, an invertebrate first intermediate host (commonly an aquatic gastropod), and a second intermediate host (a variety of invertebrates or vertebrates depending on the trematode species) which carries the encysted metacercarial stage (Fig. 1.2) (Johnson et al. 2004). The life cycle begins when parasite eggs are deposited into the aquatic environment from the definitive hosts (often birds or mammals) where they subsequently develop and hatch into motile miracidia (Johnson et al. 2004). Miracidia penetrate and infect the snail host, metamorphosing into sporocysts or rediae depending on the trematode species and begin development and multiplication through asexual reproduction (Cort 1954). Sporocysts and rediae are localized to the digestive gland-gonad (DGG) complex in snail hosts, which is the posterior section of the snail (Baudoin 1975). Within infected snails, the upper section of the snail (head/foot) region remains free of trematode tissue. Both sporocysts and rediae serve as sacs for cercariae development although they differ in their functionality (Cort 1954). Rediae 
contain a muscular pharynx and digestive tract that allow them to feed on host tissue, typically the gonads, as well as appendages for movement within the snail host (Fig. 1.3d, e) (Diconza and Basch 1975; Leung and Poulin 2011; Galaktionov and Dobrovolskij 2013). In contrast, sporocysts absorb host nutrients through their tegument (Leung and Poulin 2011). Once the cercariae have reached maturity, they exit the rediae or sporocysts and emerge from the snail into the aquatic environment (Fig. 1.3a, c). Once they have emerged, these non-feeding cercariae rely on their own energy reserves which are made up of the nutrients they acquired from their snail hosts and typically live $\sim 24 \mathrm{~h}$ (Lafferty et al. 2008). When they have located a suitable second intermediate host, they penetrate and encyst to become metacercariae (Johnson et al. 2002). The

life cycle is completed with the consumption of the second intermediate host containing infective metacercariae by the final definitive host which then develop into adult worms (Johnson et al. 2004).

\section{Cercariae Biomass in Aquatic Ecosystems}

The primary goal of the free-living stage cercaria stage is to infect their secondary intermediate hosts. However, a large portion of parasites are unsuccessful and remain suspended in the water column or deposited in the sediment (Orlofske et al. 2015). These parasites contribute substantially to the biomass of freshwater ecosystems (Kuris et al. 2008; Thieltges et al. 2008; Lambden and Johnson 2013; Preston et al. 2013). Parasitized snails emit cercariae daily into the aquatic environment at rate of between 14 and 1660 cercariae/infected snail/day (Preston et al. 2013). This rate is substantial when estimating the annual biomass of cercariae in a typical freshwater pond. For example, Preston et al. (2013), found that Ribeiroia ondatrae infected snails had the largest annual cercarial biomass in a pond in California, with a total biomass of $156 \mathrm{mg} \mathrm{m}^{2} \mathrm{yr}^{-1}$ (Preston et al. 2013). Soldánová et al. (2016) estimated that 4.65 tons of cercariae are emitted annually into a typical freshwater pond; equivalent in mass to a large Asian elephant! Kuris et al. (2008) quantified free-living cercariae in three California estuaries and found that the annual biomass was 3 to $10 \mathrm{X}$ that of the winter bird biomass, a top ecosystem predator. This indicates that parasite biomass is comparable to several other major groups of free-living animals and larger than that of some of the principal top predators in these estuaries (Kuris et al. 2008). Additionally, Kaplan et al. (2009) estimated the annual production of cercariae to be $5 \mathrm{~g} \mathrm{~m}^{-2} \mathrm{yr}^{-1}$ at the Carpinteria Salt Marsh which would support 2-3\% of the total energetic demands of the fish community. The realization that cercariae are prominent contributors to aquatic biomass and 
productivity highlights both their ability to affect food web properties and their potential to contribute to the energy and nutrient transfers in these ecosystems. The importance of their role in these systems becomes even more apparent when considering their potential to serve as prey items for predators at higher trophic levels.

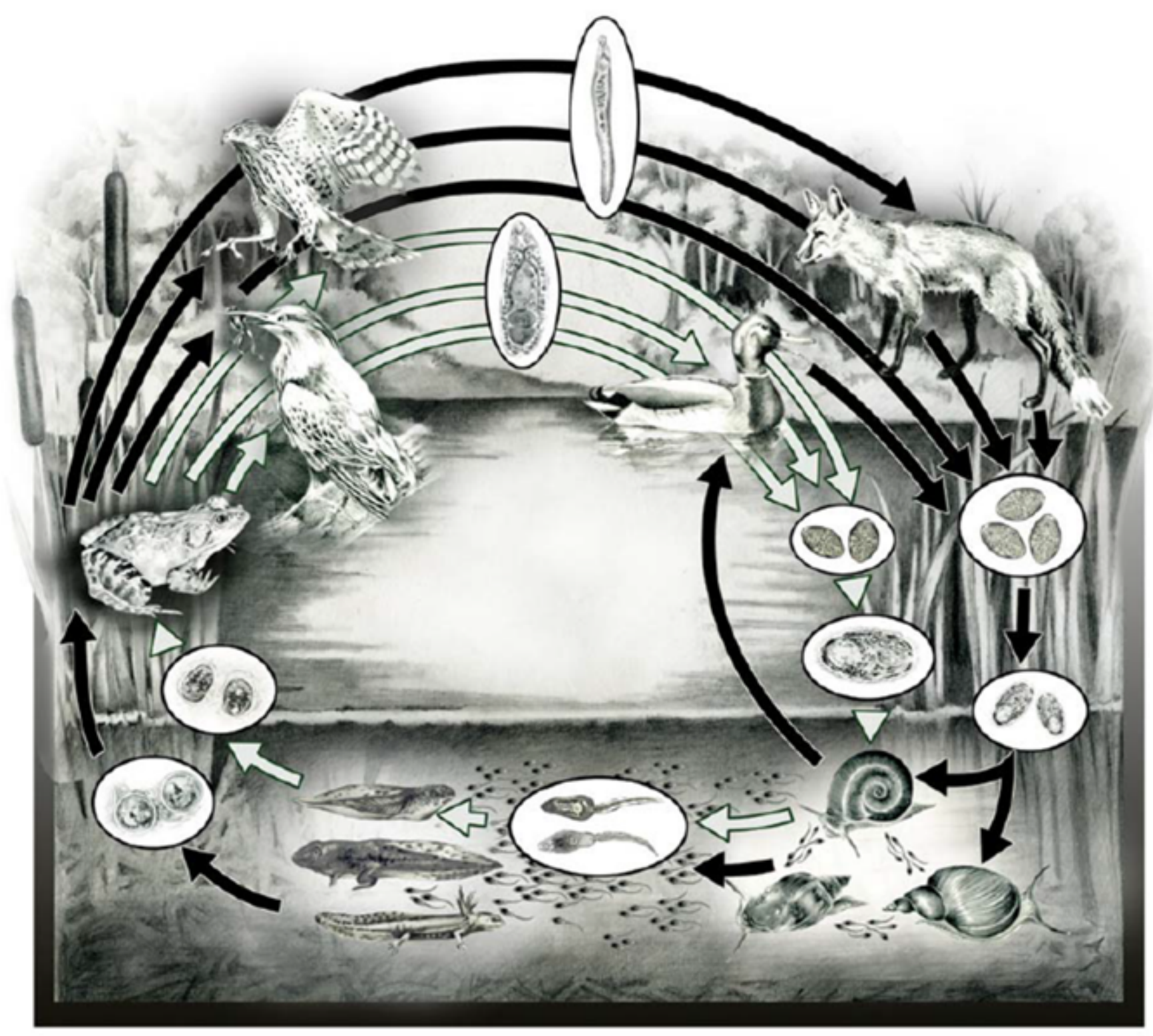

Figure 1.2. Life cycle of the trematode parasite Ribeiroia ondatrae and echinostome-related genera (Koprivnikar and Johnson unpublished). 

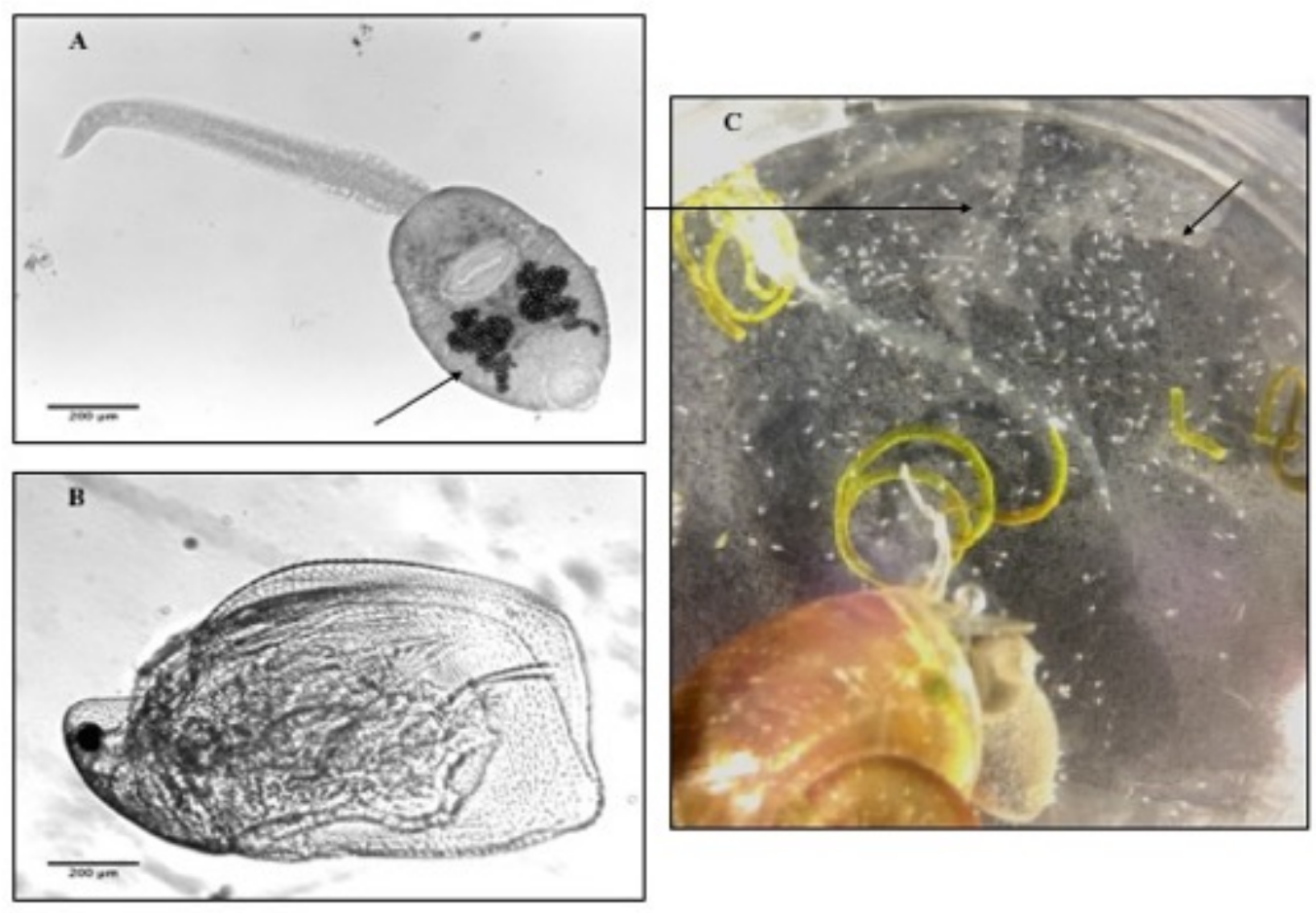
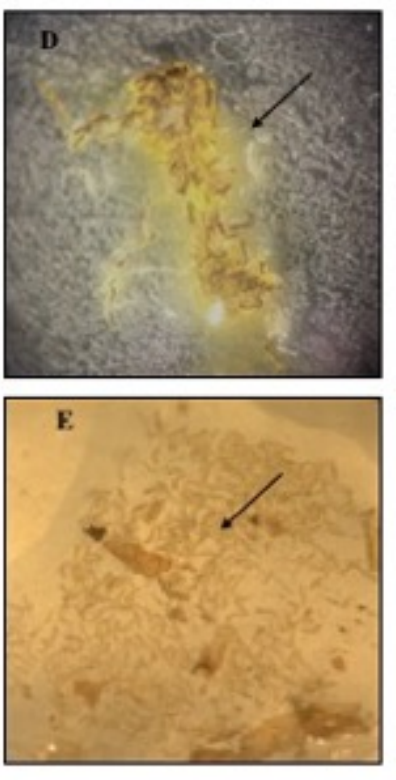

Figure 1.3 a-e. A. Ribeiroia ondatrae cercariae taken using light microscopy. The small arrow indicates esophageal diverticula a defining feature of Ribeiroia sp., scale bar is $200 \mu \mathrm{m}$. B. Daphnia pulex taken with light microscopy shows comparison to cercariae for size, scale bar is $200 \mu \mathrm{m}$. C. Helisoma trivolvis snail (bottom left) emitting Ribeiroia ondatrae cercariae (small and large black arrow) into treated water, taken with dissecting microscope. D. Ribeiroia ondatrae rediae from infected Helisoma trivolvis snail, arrow pointing to rediae, taken with camera. Estimated magnification of one organism $1200 \mu \mathrm{m}$. E. Ribeiroia ondatrae rediae from infected Helisoma trivolvis snail, arrow pointing to rediae, taken with camera. Estimated size of one organism $800 \mu \mathrm{m}$. D and $\mathbf{E}$ demonstrate the potential variability in rediae appearance. 


\section{Consumption of Parasites in Aquatic Systems}

Cercariae are analogous to meroplankton (planktonic larvae of benthic organisms) that typically fall in the size range of mesoplankton (0.2-2 mm) (Morley et al. 2012). Importantly, cercariae overlap in size with many zooplankton and zoobenthos fauna, ranging considerably in size from 250 to $1200 \mu \mathrm{m}$ (Fig. 1.3 a, b) (Pereira et al. 2013; Orlofske et al. 2015). Daphnia range in size from $\sim 750$ to $1500 \mu \mathrm{m}$ (Ebert 2005), the common calanoid copepod Diaptomus minutus is $\sim 1000 \mu \mathrm{m}$ long (Bogdan and Gilbert 1984) and the commercially important rotifer, Brachionus calyciflorus, used in aquaculture as food for fish larvae, ranges in size from 175 to $213 \mu \mathrm{m}$ (Xi et al. 2002).

Due to their size, cercariae are preyed upon by a range of benthic and planktonic freshwater animals including bryozoans, rotifers, cladocerans (Bythothrephes and Ceropagis), copepods, oligochaetes, Hydra sp., the aquatic larvae of damselflies and dragonflies as well as planktivorous and predatory fish (Tokobaev et al. 1979; Stoddart 1982; Schotthoefer et al. 2007; Thieltges et al. 2008; Kaplan et al. 2009; Catania et al. 2016). In addition, cercariae that are not consumed by predators still contribute to the flow of energy as detritus through the bacterial decomposition cycle (Thieltges et al. 2008). Therefore, cercariae comprise a significant proportion of the zooplankton community, and thus potentially serve as an important food source for consumers (Morley 2012).

Lafferty et al. (2006) estimated that $44 \%$ of the links in an estuarine food web involve predation on parasites. For example, Kaplan et al. (2009) found that most estuarine fish, including guppies and African killifish, will consume cercariae (Schistosoma mansoni) in a lab setting and that cercariae were found in the guts of most fish species in Southern California, indicating that predation is a regular event. Orlofske et al. (2015) performed numerous laboratory experiments to investigate the consumption of parasites by damselfly nymphs, as well as adult and juvenile fish in light and dark conditions across a range of predator body lengths. They found that parasites were effectively consumed (consumption rate 50-70\%) by all prey but most effectively by juvenile fish in ambient light conditions (Orlofske et al. 2015). Catania et al. (2016) determined that the larval odonate, Leucorrhinia intacta, (Hagen 1861) readily attacked and consumed cercariae even when provided with Daphnia sp. as an alternative food source. Although studies have demonstrated that the predation of cercariae by freshwater organisms is common and deliberate, the growth and condition of the predators consuming cercariae as an 
alternative food source has yet to be investigated. It is imperative that the nutritional value of cercariae is determined as their consumption likely represents an important and unrecognized trophic link that supplies substantial biomass, energy, and nutrients up the food chain (Johnson et al. 2010).

Thus, although cercariae have been found to contribute substantially to zooplankton and detritivore communities, and their immense biomass gives them the ability to contribute significantly to food web energetics as nutritionally viable prey items, their nutritional value is currently unknown (Johnson et al. 2010). For instance, histochemical analysis has revealed that cercariae contain visible storage lipid reserves, however quantification of the amounts and types of lipids is still lacking (Fried et al. 1998c).

\section{Lipids as Drivers of Ecosystem Productivity}

Understanding the processes that regulate the efficiency of energy and nutrient transfer and therefore food-quality is a critical challenge confronting the study of aquatic food webs (Brett and Müller-Navarra 1997). A key nutrient used to assess food quality and subsequently somatic growth and reproduction in freshwater ecosystems are essential fatty acids (EFA; Kainz et al. 2004; Arts et al. 2009). Fatty acids are characterized by an aliphatic chain with a carbonyl group at one end and a methyl group on the opposite end. They can either be saturated, containing no double bonds in the chain, or unsaturated, with different numbers of double bonds. Unsaturated FA are synthesized from saturated FA through the introduction of double bonds at specific positions by enzymes called desaturases (Mayes et al. 2003). A monounsaturated FA (MUFA), has one double bond, and a polyunsaturated FA (PUFA) or a long-chain polyunsaturated FA (LC-PUFA) has more than one double bond. LC-PUFA are a subsample of PUFA with twenty or more carbons in the aliphatic chain.

Lipids are a global energy currency because of their extensive physiological roles in aquatic and terrestrial biota. It is generally thought that eicosapentaenoic acid (EPA, 20:5n-3) and docosahexaenoic acid (DHA, 22:6n-3) are the most important EFA, as they play a vital role in the regulation of cell membrane properties, serve as precursors for crucial animal hormones and have a critical role in the development and function of neural and ocular tissue (Shul'man and Yuneva 1990; Innis 2007; Brenna et al. 2009; Farooqui 2009; Parrish 2009; Land 2009; Simopoulos 2011). EFA are designated essential since they cannot be biosynthesized in sufficient quantities to meet optimum physiological needs (Arts et al. 2001; 2009). They are 
almost exclusively synthesized de novo by plants; however, most organisms have some capacity to convert ALA ( $\alpha$-linoleic acid, 18:3n-3) to EPA and DHA (Arts et al. 2009). Despite this ability, the conversion is inefficient within all organisms and is therefore not sufficient to support or maintain optimal growth rates. Therefore, organisms are required to obtain these FA, preformed, from direct dietary sources (Arts et al. 2009).

Some algal taxa (e.g.,, diatoms, dinoflagellates, cryophytes) produce significant amounts of EPA and DHA (Brett and Müller-Navarra 1997; Kainz et al. 2004; Arts et al. 2009; Gladyshev et al. 2013; Hixson et al. 2015; Galloway and Winder 2015), and these LC-PUFA are consumed, and generally selectively retained, by other aquatic organisms at higher trophic levels (e.g.,, zooplankton, benthic invertebrates, molluscs, fish) (Dalsgaard et al. 2003; Kainz et al. 2004; Lands 2009; Taipale et al. 2013). It is thus vital to understand the dynamics of LC-PUFA at the base of aquatic food webs as they are key drivers of aquatic ecosystem productivity (MüllerNavarra 1995; Müller-Navarra et al. 2000). For example, zooplankton were found to only achieve optimal growth rates when given a direct dietary source of LC-PUFA (Jones et al. 1979; Kanazawa et al. 1979; Waldock and Holland 1984), and a very strong correlation was found between EPA content of phytoplankton species and their food-quality for zooplankton and other predators (Müller-Navarra 1995). Additionally, the growth and egg production of Daphnia sp. was also predicted by the absolute concentration of EPA of the primary producer, and showed a much higher correlation than C, N and P where $\mathrm{N}$ and $\mathrm{P}$ were not limiting (Müller-Navarra 1995; Müller-Navarra et al. 2000). This led to the conclusion that EPA is a limiting resource in aquatic ecosystems.

Lipids are the primary energy currency in aquatic ecosystems and are essential to the health and survival of all invertebrates and vertebrates (Arts et al. 2001, 2009). It is known that EPA is critical for optimal organism growth and productivity within aquatic food webs, and it is thus essential to identify and quantify the potential sources. Equally important is the identification of new sources of DHA, as this biomolecule is essential for the development of vertebrate brains and eyes. EFA derived from the planktonic food web, and specifically through zooplankton, are an important link for EFA movement from algae to higher trophic levels (e.g., fish).

Cercariae are currently an undefined prey item in terms of their nutritional value for zooplankton, insect larvae, and planktivorous and predatory fish (Stoddart 1982; Tokobaev et al. 1979; Schotthoefer et al. 2007; Thieltges et al. 2008, Kaplan et al. 2009; Catania et al. 2016). 
Cercariae are in the size range of typical zooplanktonic prey (e.g., Daphnia sp.) and they lack an exoskeleton, further highlighting their suitability to serve as nutrient rich repositories of energy and nutrients for an array of predators (Ebert 2005), and may perhaps even serve as another important pathway by which EFA may be transferred to higher trophic levels. Preliminary analyses indicate that they contain lipids, and therefore, there exists the potential for them to provide a substantial source of EFA to drive aquatic productivity. This is because cercariae feed solely on the tissue of their hosts, such as planorbid snails in freshwater, whose diet consists primarily of algae (Oudejans and Van der Horst 1974; Lombardo et al., 2002) some of whom contain EPA and DHA. The composition of host snail tissue has been found to reflect their diet, as seen in an experiment where Helisoma snails (planorbids) were fed leaf lettuce which resulted in high levels of $\alpha$-linoleic acid, the most abundant fatty acid in leaf lettuce (Oudejans and Van der Horst 1974; Fried et al. 1992). EPA and DHA are synthesized and present in high concentrations in some algal taxa (e.g., diatoms, dinoflagellates, cryophytes) suggesting that snail host tissue, and more importantly, cercariae have the potential to contain these essential nutrients.

Not only is the quantification of the nutritional quality of cercariae essential to aquatic food webs, it also has significant implications for terrestrial food webs. High connectivity exists between freshwater and terrestrial landscapes and particularly in wetlands where sources of LCPUFA are transferred, by a variety of vectors (e.g., emerging insects) from aquatic to terrestrial landscapes (Gladyshev et al. 2009; 2013; Hixson et al. 2015). The distribution, production and abundance of LC-PUFA in aquatic ecosystems differ from terrestrial ecosystems, with a stronger prevalence of EPA and DHA in aquatic organisms (Hixson et al. 2015). Therefore, aquatic freshwater organisms provide an essential source of FA through the production of n-3 LC-PUFA which are required for the health of terrestrial organisms and humans (Arts et al. 2001; Hixson et al. 2015). Cercariae have the potential to contribute to the transfer of these LC-PUFA subsidies from wetlands to terrestrial consumers via various indirect dietary mechanisms (Gladyshev et al. 2009, 2013). For example, cercariae are predated and consumed by emergent larval insects, which have both an aquatic and terrestrial life stage. These organisms transfer LC-PUFA obtained from the aquatic ecosystem to the terrestrial landscape (Martin-Creuzburg et al. 2017). Additionally, cercariae are consumed by fish, potentially providing terrestrial piscivores (e.g., eagles, herons, racoons, bears etc.) with additional essential nutrients. The quantitative 
identification of cercariae lipid subsidies would allow for a more comprehensive understanding of the nutrient and energy flow of essential nutrients in both aquatic and terrestrial landscapes.

\section{Cercariae Composition}

Early investigations of their composition have been largely qualitative and determined that they contain lipids and are glycogen-rich, suggesting that they are nutritious (Moore and Halton 1975; Diconza and Basch 1976; Fried et al. 1998a; Furlong et al. 1992; Nanduri et al. 1991; Xu et al. 1994). These efforts have primarily employed the use of Oil Red-O (ORO), and Nile Blue Sulphate as means to stain and visually detect the presence of neutral lipids and FA in rediae, cercariae and sporocysts of various trematode species. Moore and Halton (1975) histochemically observed the presence of scattered FA in the rediae of Fasciola hepatica, as well as neutral lipid deposits and low FA levels in the cercariae. Diconza and Basch (1976) observed diffused lipids in the mother and daughter sporocysts of $S$. mansoni. Fried et al. (1998b) investigated the differences in lipid content of various cercariae species, observing a range from none found in $Z$. lunata, to ORO-positive droplets in the excretory system of Ribeiroia sp. Furlong et al. (1992) used fluorescently-labeled liposomes in the post-penetration stage known as schistosoma of Schistosoma mansoni to demonstrate the incorporation of phospholipids and the accumulation of lipids in specialized cells called cytons. Additionally, Furlong et al. (1995) used a fluorescent probe to tag FA in S. mansoni cercariae, and observed diffused fluorescence and discrete lipid droplets throughout the organism. They concluded that the majority of lipids are in the form of diglycerides (65\%), monoglycerides (8\%) and phospholipids (10\%), however, they did not specifically identify the FA present (Furlong et al. 1995). Xu et al. (1994) and Nanduri et al. (1991) also investigated glycolax, the carbohydrate-rich, glycogen-like material which covers the exterior of schistosomal cercariae. They identified that the glycolax complex contains fucose, galactose, amino sugars and glucose (Nanduri et al. 1991; Xu et al. 1994). Studies have also suggested that cercariae tails have significant glycogen stores to supply them with energy for survival. Studies have found that cercarial survival rates are dependent on the finite glycogen reserves that have been identified in the tail (Anderson and Whitfield 1975; Pechenik and Fried 1995; McCarthy 1999). These studies suggest that the rediae and cercariae of multiple species of trematode parasites are lipid and glycogen rich.

More recently, quantitative efforts using high-performance thin-layer chromatography (HPLC) have quantified the free sterols in trematodes but have focused less on the quantification 
of other lipids. Table 1.1 shows the free sterol and total free fatty acids contents on a ng per cercariae basis for two digenean trematode species, Zygocotyle lunata (Marsit et al. 2000a) and Schistosoma mansoni (Schariter et al. 2002). Only the free sterol content of Echinostoma caproni (Marsit et al. 2000b) and Echinoparyphium sp. (Muller et al. 1999) are shown in Table 1.1, as they did not perform more detailed FA analysis. Although total FA contents have been quantified for the two species, more detailed identification of the individual FA is still lacking. Marsit et al. (2000a) used visual chromatographs to determine that the most abundant lipid fractions in $Z$. lunata were free sterols and free FA in the larval stages and triacylglycerols in the metacercarial and redial stages. Schariter et al. (2002) quantified the FA content for S. mansoni, and from visual chromatograms determined that there was a large and fast-moving lipid zone, with the most abundant phospholipids being phosphatidylcholine (PC) and phosphatidylethanolamine (PE). Both Marsit et al. (2000a) and Schariter et al. (2002) failed to identify the individual FA present in cercariae. Sterol content was found to vary greatly between the different cercariae taxa, and this is not surprising considering the extreme variation in their morphology. Similar variation is also expected to be found in FA content among cercariae of different species.

Other researchers have examined the effects of parasite infection on the lipid composition of the host snail tissue (Fried et al. 1989, 1990, 1993; Thompson 1987; Bandstra et al. 2006). Fried et al. $(1990,1993)$ found that $H$. trivolis gastropods infected with Echinostoma trivolvis parasites had elevations in both total lipids and major neutral lipid fractions in the snail digestive gland; the area where the parasite rediae are located. They used gas-liquid chromatography (GC) to determine the FA composition of the digestive gland-gonad (DGG) complex of infected tissue, of rediae freed from the DGG, of uninfected DGG and of 41-day old adult worms grown in hamsters. They found that EPA was the most abundant FA in the pentaene group, followed by DHA in all of the samples, ranging from 1\% EPA in the uninfected DGG to 5.5\% of all FA in adult worms. These results were similar to those reported by Thompson (1987) for Biomphalaria glabrata infected with $S$. mansoni; however, they contradict previously reported findings by Fried et al. (1989), who found a reduction in the triacylglycerol (TAG) fraction of the digestive gland-gonad complex of infected snails matched with controls. These results indicate that lipids, specifically EPA, may be present in rediae sacks, which contain cercariae at various stages of development. However, the literature still lacks a detailed parasite lipid composition, which is a necessary component in determining their viability as a nutrient-rich food source. 
Table 1.1: Free sterol and fatty acid composition of cercariae from various trematode parasite species.

\begin{tabular}{|c|c|c|c|}
\hline Component Quantified & Species & ng per Cercaria & Reference \\
\hline Free Sterols & Zygocotyle lunata & 120 & Marsit et al. 2000 \\
\hline Free Sterols & Echinostoma caproni & 0.53 & Marsit et al. 2000 \\
\hline Free Sterols & Echinoparyphium sp. & 22 & Muller et al. 1999 \\
\hline Free Sterols & Schistosoma mansoni & 3.51 & Marsit et al. 2000 \\
\hline Total Fatty Acids & Zygocotyle lunata & 160 & Marsit et al. 2000 \\
\hline Total Fatty Acids & Schistosoma mansoni & 4.6 & \\
\hline
\end{tabular}

\section{Thesis Goals}

The goal of this thesis was to investigate the nutritional composition of trematode parasites and their hosts, focusing on their ability to serve as nutrient-rich prey items in aquatic ecosystems. Cercariae fall in the size range of zooplankton, are released in immense numbers into the aquatic ecosystem and are consumed by a variety of aquatic invertebrate predators. I quantified the neutral energy reserve lipid and EFA composition of trematode parasites and assesed their ability to transfer these nutrient-rich subsidies to their predators. For the majority of my thesis work I focused on Ribeiroia ondatrae cercariae as my model trematode infection. This species was chosen due to: a) its large size, which rendered amassing enough individuals for lipid extraction samples logistically feasible, b) the availability of rigorous estimates of their biomass and production in numerous systems, c) their vaste distribution in North America and d) their high prevalence in certain California ponds where they are implicated in causing amphibian deformities (Johnson and McKenzie 2009). Addtionally, larval odonates transfer EFA from the aquatic to the terrestrial landscape, and I wanted to investigate the potential for $R$. ondatrae cercariae to contribute to this essential subsidy. This work contributes to a body of literature suggesting the need to add cercariae to food webs as they may provide substantial nutrients in aquatic ecosystems. Despite these suggestions, there has yet to be work investigating this 
potential. My thesis contributes novel, quantitative, results to the effort of adding parasites to food web models by demonstrating the value and contribution of cercariae in the context of essential lipids, bolstering the case for their inclusion as integral members.

Chapter 2 - How does the quantity and distribution of neutral lipids differ among cercariae species?

To investigate this question, I collected cercariae from 5 trematode species/types: Ribeiroia ondatrae, Echinostoma trivolvis., Haematoloechus sp., armatae-type and strigeid-type cercariae. Fluorescently stained images were analyzed using both qualitative and quantitative methods to determine the differences in neutral energy reserve lipid distribution among the species. Energy reserve lipids were chosen for analysis since lipids are considered to be the primary energy currency in aquatic ecosystems and are essential for the development and health of invertebrates and vertebrates. A semi-quantitative binning technique was used to group species based on visual lipid observations as well as a quantitative method where fluorescence values were calibrated to a concentration curve. These analyses provided information on the presence of lipids in cercariae, how their composition and distribution differ among species and provided an estimate of the quantity of lipids present in numerous cercariae species. Additionally, I ran a fatty acid profile of $R$. ondatrae cercariae to determine if they contain EFA and significant EPA and DHA. These results contribute vital information on the prescence, distribution, and quantity of energy reserve lipids and EFA in cercariae. Both proposed methods allow for fast and rigourous quantification of cercariae lipid and EFA profiles, which is an essential step in determining the contribution of cercaria to nutrient and energy flows in aquatic food webs.

Chapter 3 - Does Riberioia ondatrae infection affect the fatty acid composition of snail hosts?

I ran a fatty acid profile of $R$. ondatrae infected and uninfected $H$. trivolvis snails. I hypothesized that $R$. ondatrae cercariae would contain EFA and significant amounts of EPA and DHA due to the consumption of their snail host tissue, whose diet consists primarily of algae. I also hypothesized that the infected snail tissue would contain higher amounts of total lipids then uninfected tissue, due to previous literature findings. Additionally, I estimated the quantity of EFA from parasites in a typical pond using fatty acid analysis coupled with estimates of total yearly cercariae biomass and output within a pond ecosystem. Thus, this chapter compliments 
and expands on previous studies, especially given opposing findings of the effects of parasite infection on host snails. By investigating the effects of $R$. ondatrae infection within $H$. trivolvis snails I am helping to deduce the drivers of parasite-host lipid relations. This has far-reaching consequences within food webs as well as effects on host populations and distributions.

Chapter 4 - How nutritionally viable are cercariae as a food source for aquatic organisms? Are essential fatty acids (EFA) from cercariae retained in aquatic insect larvae from species that emerge onto terrestrial landscapes as adults (i.e. aquatic to terrestrial subsidies)?

Here, I assessed the viability, growth and changes in lipid composition of the larval odonate, L. intacta. Larval dragonflies were separated into two treatments and fed $R$. ondatrae or an equivalent biomass of Daphnia spp. They were then monitored for growth and analyzed for FA profiles after 5 weeks of feeding. I hypothesized that growth rates and lipid profiles would be similar in $R$. ondatrae and Daphnia spp. fed dragonfly larvae, suggesting that cercariae are a potentially equally viable food source and contribute to zooplankton communities. I also hypothesized that EFA would be retained in larval dragonflies fed $R$. ondatrae, and which would confirm that lipids from cercariae are trophically transferred through aquatic insects and contribute to key aquatic and terrestrial lipid subsidies. As aquatic insects are one of the most important pathways through which EFA are transferred to the terrestrial landscape, the potential for cercariae to contribute to that route through larval odonate consumption would indicate their necessity in food web theory. 


\title{
CHAPTER 2: LIPID CONTENT AND FATTY ACID ANALYSIS OF TREMATODE PARASITE CERCARIAE
}

Keira M. Mckee, Janet Koprvnikar, Pieter T. J. Johnson and Michael T. Arts.

Sections of this chapter and Chapter 4 are in preparation to be submitted to Oecologica.

\begin{abstract}
Trematode parasite cercariae contribute immense biomass within aquatic ecosystems and are commonly and deliberately consumed by a variety of aquatic invertebrates. As such, they may play an integral role in supplying the aquatic food web with nutrient-rich subsidies. Despite this, quantitative evidence indicating their nutritional value is currently lacking. Energy reserves (e.g., high capacity energy storage molecules, such as triacylglycerols = TAG) and fatty acids are both powerful indicators of an aquatic organism's caloric and nutritional food value, respectively. Essential fatty acids (EFA) are also integral to the development and health of invertebrates and vertebrates. I developed a quantitative binning technique to rapidly assess the neutral energy reserve lipid of 5 trematode cercariae species/types and also created a TAG concentration curve to derive an estimate of the total neutral energy reserve lipids in an individual cercaria. The binning technique successfully detected differences among cercariae types and provides a reliable and fast method for cercarial lipid store comparisons. Additionally, a fatty acid profile of Ribeiroia ondatrae cercariae was obtained, with a specific focus on detecting and quantifying essential fatty acids (EFA). I found that cercariae were lipid-rich (i.e. had significant quantities of total lipids) and that they contained EFA, including both EPA and DHA. These results contribute vital information on the presence and distribution of lipids and EFA in cercariae. Both proposed methods allow for fast and rigourous quantification of cercariae lipid and EFA profiles, which is an essential step in considering their contribution to energy and nutrient flows in aquatic food webs.
\end{abstract}




\section{Introduction}

Energy reserves (e.g., high capacity energy storage molecules, such as triacylglycerols = TAG) in aquatic organisms affect their survival and fitness. This stored energy reflects feeding success (Holm and Shapiro 1984; Gallager et al. 1986), allows organisms to survive periods of fasting (Pond 1981), and is related to future reproductive potential (Goulden et al. 1982). The high energy content of zooplankton also serves as a powerful indicator of their caloric food value for planktivorous larval fish (Arts and Sprules 1989). Another important metric used to assess the composition of zooplankton in this context are essential fatty acids (EFA), as they affect somatic growth and reproduction of both invertebrates and vertebrates in aquatic ecosystems (Brett and Müller-Navarra 1997; Parrish et al. 1999; Kainz et al. 2004). Most animals cannot synthesize EFA in sufficient quantities to meet dietary needs, therefore the identification of dietary sources (including heretofore unrecognized ones) is vital (Arts et al. 2001).

The long-chain polyunsaturated fatty acids (LC-PUFA), including eicosapentaenoic acid (EPA; 20:5n-3), docosahexaenoic acid (DHA; 22:6n-3) and arachidonic acid (ARA; 20:4n-6), are considered important as they have vital bioactive capabilities (Simopoulos 1999; Arts et al. 2001; Innis 2007; Parrish 2009). The shorter-chain FA, including alpha-linolenic acid (ALA; 18:3n-3) and linolenic acid (LNA; 18:2n-6), are also considered EFA as they can be converted into the longer chain $n-3$ and $n-6$ constituents through a series of elongation and desaturation reactions (Kanazawa 1979). EPA and DHA are considered to be among the most important LCPUFA as they play vital roles in the regulation of cell membrane properties, serve as precursors for crucial animal hormones, and underpin the healthy function of neural and ocular tissues (Shul'man and Yuneva 1990; Innis 2007; Brenna et al. 2009; Farooqui 2009; Parrish 2009; Lands 2009; Simopoulos 2011). EPA is produced in high quantities at the base of the aquatic food webs in some algal taxa (see Chapter 1), and serves a vital role in ecosystem productivity and organism health (Arts et al. 2009). Additionally, EPA is limiting; in cases where nitrogen, and especially phosphorus are not, Daphnia growth rates declined when the EPA content of seston was reduced due to changes in algal species composition (Müller-Navarra 1995; MüllerNavarra et al. 2000). For example, in eutrophic conditions Daphnia growth suffers as cyanobacteria blooms dominate the phytoplankton community. This is because cyanobacteria contain little to no EPA (20:5n-3), which has been shown to limit Daphnia growth and reproduction (Müller-Navarra et al. 2000), ultimately affecting ecosystem productivity. In order 
for EPA produced at the base of the aquatic food web to reach higher trophic level organisms, it must be trophically-transferred. Zooplankton are recognized as a crucial link for the trophic transfer of essential nutrients (including bioactive lipids) from the base of aquatic food webs to higher trophic level organisms (Müller-Navara 1995). Therefore, the identification of novel mechanisms (i.e. other potential organisms) through which EFA, including EPA and DHA, can be trophically transferred is fundamental to understanding aquatic ecosystem dynamics.

The life cycle of trematode (flatworm) parasites is complex with multiple developmental stages and intermediate hosts. Cercariae, a free-living stage of the trematode life cycle, are released in high numbers from their first intermediate hosts (commonly aquatic gastropods) into the aquatic environment, where they attempt to locate their second intermediate host (see Chapter 1, Fig. 1.2) (Kuris et al. 2008; Kaplan et al. 2009; Preston et al. 2013; Soldánová et al. 2016). Cercariae have recently been suggested as analogous to zooplankton in their ability to serve as prey and provide nutrients to higher trophic level predators (Morley 2012, see Chapter 4). Despite this, much less is known, relative to zooplankton, in terms of their nutrient composition.

Prior to emergence, cercariae develop within sporocysts or rediae which consume hostderived nutrients, either through absorption in the former, or direct consumption through a mouthpart in the latter (Choubisa 2008). Importantly, cercariae solely receive nutrients from their host, and rely on these once emitted into the aquatic environment because they are non-feeding. Recently, this free-living stage has garnered attention for its ability to alter food web dynamics through its immense collective biomass, but also in the ability of cercariae to serve as prey for a variety of aquatic invertebrates and vertebrates (Schotthoefer et al. 2007; Kaplan et al. 2009; Orlofske et al. 2012, 2015; Catania et al. 2016). As such, cercariae may represent a significant, but currently underappreciated, nutrient source in aquatic ecosystems (Johnson et al. 2010). Despite the identification of these newfound roles, cercariae currently remain absent from food web models, despite repeated calls for their inclusion (Marcogliese and Cone 1997; Lafferty et al. 2006; Lafferty et al. 2008; Byers 2009; reviewed by Sukhdeo 2012). To bolster the case for their inclusion, and to quantify their contribution to nutrient fluxes within aquatic ecosystems, it is necessary to first determine the nutritional composition of cercariae.

To date, studies investigating the composition of cercariae have largely focused on qualitative histochemical analyses, which have demonstrated extreme morphological variation in cercariae 
from different taxa that drives differences in lipid and sterol composition (Schariter et al. 2002). Cercariae contain different body structures and organs; however, type-specific differences such as the presence and absence of specialized organs which may secrete lipids, sterols (etc.) for various functions, give rise to differences in nutrient composition (Schariter et al. 2002). Although cercariae are non-feeding, they typically contain many of the structures present after transformation to the adult worm stage if successfully transmitted. Thus, basic cercarial internal morphology consists of an alimentary system including a mouth surrounded by an oral sucker, pre-pharynx, muscular pharynx, oesophagus, oesophageal glands, and two intestinal caeca (Frandsen and Christensen 1984). The excretory system consists of an excretory bladder and a system of primary, secondary and tertiary collecting vessels on either side of the body, where the tertiary vessels terminate in flame-cells which are equipped with capillaries (Frandsen and Christensen 1984). Additionally, three main types of glands exist: mucoid gland cells, cystogeneous gland cells, and penetration gland cells each of which secrete substances that aid in the penetration of the host tegument (Frandsen and Christensen 1984). All or some of these features may be present or missing based on the species of cercariae because different tissues have different compositions, giving rise to extreme variations in morphology and chemical composition. Additionally, different cercariae species may acquire nutrients through different mechanisms (i.e. rediae vs. sporocysts feeding on different host tissues), and cercariae may have different biosynthetic capabilities (e.g., some species may be better adapted to convert shorter chain FA into longer chain constituents). Finally, the effect of environment (e.g., temperature) may have a role in the total neutral energy reserves and FA profile that I observe in Ribeiroia ondatrae cercariae, as seen in other aquatic species (Widdows and Bayne 1971, Schlechtriem et al. 2006).

Histochemical investigations into the composition of cercariae have identified the presence of lipids, glycogen and sterols, and that the levels of these compounds vary throughout the trematode life cycle (see Chapter 1). Visual indexes to estimate the energy reserves of aquatic invertebrates, such as Daphnia sp. and other zooplankton, have been previously developed to investigate individual differences in lipid energy reserves (Goulden and Hornig 1980; Tessier and Goulden 1982; Holm and Shapiro 1984; Tessier et al. 1983; Arts and Evans 1991). The use of microscopy to measure visible energy reserves in copepods, cladocerans, and invertebrate larvae has also included the estimation of lipid droplet volume by assuming either spherical (Arts 
and Sprules 1988; Kuosa and Gyllenberg 1989) or prolate spheroidal shapes (McLaren et al. 1989; Arts and Evans 1991). However, most of these early studies did not compare the microscopic measurements of lipid volumes to an independent (e.g., chemistry-related) measure of energy reserves, such as the later comparison done with an Iatroscan TLC-FID by Arts and Evans (1991). Arts and Evans (1991) thus developed a method for measuring visible lipid energy reserves in copepods, and highlighted the reliability and comparability of their approach by comparing it to both microgravimetric (bulk lipid measurement) and quantitative flameionization measurement (TAG) techniques.

Despite previous histochemical investigations of cercariae, no such quantitative estimation techniques have been developed that are comparable to those used for zooplankton even though the transparent tegument of cercariae, and the fluorescent pigment associated with stained neutral lipids (Green 1957), similarly allow for the direct visual observation of lipid stores (Arts and Evans 1991). While quantitative efforts to analyze the total lipid content of cercariae have investigated two morpho-types, detailed identification of individual lipid classes in cercariae is still lacking. Marsit et al. (2002a) utilized TLC to determine that the most abundant lipid fractions in Zygocotyle lunata cercariae were free sterols and neutral lipids. Additionally, Schariter et al. (2002) quantified lipid classes in Schistosoma mansoni and determined that there was a large and fast-moving lipid zone, with abundant neutral lipids, and phosphatidylcholine (PC) and phosphatidylethanolamine (PE) making up the majority of phospholipids. However, neither of these two studies identified the individual FA present in cercariae.

Here, I present an optical binning technique used in conjunction with Nile red-stain, to visualize individual cercarial neutral lipid energy stores (e.g., TAG, DAG) as a means to quantitatively compare amounts of energy reserve lipid among individual cercariae. This technique also allows for the investigation of individual differences among cercariae in their ability to store energy reserve lipids in relation to factors such as location, temperature, taxon, etc., and which cannot easily be accomplished with other techniques, e.g., Iatroscan TLC-FID, due to the tissue mass required for accurate quantification (with replication). I also provide a detailed FA profile of $R$. ondatrae cercariae, which represents the first investigation into individual FA present in any cercariae of digenean trematodes, and highlights their potential to contribute EFA to aquatic ecosystems. Although the aforementioned lipid extraction techniques are necessary for the advancement of our understanding of lipids as nutrients and energy stores 
in aquatic invertebrates including parasites, they can be difficult to perform for a number of reasons, highlighting the importance of the development of complimentary histochemical techniques. For example, lipid extraction and quantification require specialized knowledge and costly equipment which sometimes limits its use. Most important is the need for sufficient sample mass, especially when quantifying cercarial FA profiles on an absolute (i.e. mass fraction) basis by gas chromatography, and which requires the pooling of tens of thousands of cercariae for a single sample.

As EFA, such as EPA and DHA, are synthesized in high quantities, mostly by primary producers (and potentially some animals where the genes required to synthesize n-3 FA have been identified), cercariae may consume these EFA through the tissue of their herbivorous first intermediate hosts (e.g., aquatic gastropods) (Cort 1954; Brett and Müller-Navarra 1997; Kainz et al. 2004; Arts et al. 2009; Kabeya et al. 2018). Because snail tissue composition reflects that of their diet (Oudejans and Van der Horst 1974, Lombardo and Cooke 2002), it contains high quantities of EFA that may then be transferred to the developing cercariae within, as ingested snail cells have been identified in rediae tissue (Moore and Halton 1975). I thus hypothesized that cercariae likely contain significant quantities of EFA and that they therefore may be contributing substantial quantities of these key nutrients to aquatic ecosystems. By developing a quantitative histochemical method to investigate individual differences in neutral lipid energy reserves, as well as providing a FA profile for $R$. ondatrae cercariae, my work furthers our understanding of the potential contribution of the trematode infectious stage to the trophic transfer of EFA.

\section{Materials and Methods}

\section{Field Collection and Animal Care}

During June-August 2017, I collected snails in numerous freshwater ponds in northern California (USA) and Southern Ontario (CA). Aquatic snails (Helisoma trivolvis) were collected from two ponds in Pleasanton Ridge Regional Park in California (37.6154094 $\mathrm{N}$, $\left.121.8823708^{\circ} \mathrm{W}\right)$, as well as from two ponds near Guelph, Ontario $\left(43.7722028^{\circ} \mathrm{N}\right.$, $\left.080.3772306^{\circ} \mathrm{W}\right)$. A different species of aquatic snail (Lymnaea stagnalis) was also collected from two ponds near Guelph, $\mathrm{ON}\left(43.4711944^{\circ} \mathrm{N},-080.2395833^{\circ} \mathrm{W}\right)$, as well as from one pond 
near St. Catherines, ON (43.1188781 $\left.{ }^{\circ} \mathrm{N},-079.2489728^{\circ} \mathrm{W}\right)$. Snails in Ontario and California were collected using dip nets along the perimeter and within $2 \mathrm{~m}$ from the edge of each pond, and they were also collected in California by seining in the middle of the pond using a square kick-net with hemmed sides and hand-held poles (1 mm mesh, $2.5 \mathrm{~m} \mathrm{X} 1 \mathrm{~m})$. The dip net collections were performed at ten evenly distributed transects, $1 \mathrm{~m}$ in length, along the shoreline using a D-frame dip net (1.4 mm mesh size, $2260 \mathrm{~cm}^{2}$ opening) which was pulled quickly through the water at a distance of $1.5 \mathrm{~m}$ perpendicular to the shoreline (Johnson et al. 2002). Seining, using the kick-net, was conducted at randomly chosen locations within the pond (at $\sim 1$ $\mathrm{m}$ depth) with transects ranging in length from 2-10 $\mathrm{m}$.

Snails were collected and transported to Ryerson University and maintained in 13 L plastic containers containing dechlorinated tap water. The snails were screened for trematode infection using established procedures (Szuroczki and Richardson 2009). I placed individual snails into 6well tissue-culture plates; with each well containing $32 \mathrm{~mL}$ of de-chlorinated tap water, and kept them under a $60 \mathrm{~W}$ incandescent lamp for $1 \mathrm{~h}$ to stimulate emergence of cercariae. To determine the presence of $R$. ondatrae infection, snails were left overnight in a Petri dish with dechlorinated tap water and kept in the dark until they could be checked for cercariae emergence the next morning ( 7 AM), in recognition of their known nocturnal peak emergence patterns (Szuroczki and Richardson 2009). For infected snails (i.e. confirmed by cercariae emergence by inspecting wells/dishes under a dissecting microscope), the trematode genus and species (when possible) was identified using a compound microscope and identification keys (Schell 1985). Infected snails were then separated, based on the genus/species and on location of the site from where they were collected, and placed into labeled $13 \mathrm{~L}$ tubs containing dechlorinated tap water (changed every $3 \mathrm{~d}$ ). Snails were fed raw spinach leaves and they also had access to Chlamydononas sp. algae that were growing in each container (see Ch. 4). Depending on which trematode species was needed on any given day, the corresponding cercariae were collected from infected snails with a micropipette and placed into $1.5 \mathrm{~mL}$ microcentrifuge tubes filled with the same dechlorinated tap. After screening both H. trivolvis and L. stagnalis snails for trematode infections, I was able to obtain cercariae of 5 different species/types: $R$. ondatrae, Echinostoma sp., and Haematoloechus sp., as well as one each of armatae- and strigeid-types (see Schell 1985 for a description of these types). 


\section{Nile Red Staining of Cercariae}

Nile red $\left(\mathrm{C}_{20} \mathrm{H}_{18} \mathrm{~N}_{2} \mathrm{O}_{2}\right)$ has previously been used to detect neutral lipids in algal cells as well as in various cercariae morpho-types and life stages (Frandsen and Christensen 1984, Chen et al. 2009, Held and Raymond 2011, Storms et al. 2014). Nile red was used here to detect neutral lipids by preparing a stock solution after dissolving technical grade Nile red powder (N3013, Sigma-Aldrich) in acetone (1200-1-42, ACS Reagent Grade, Caledon Laboratories Ltd, Canada) to a final concentration of $1 \mu \mathrm{g} / \mathrm{mL}$. The stock solution was sealed and stored in a dark cabinet at room temperature. A $2 \mu \mathrm{l}$ aliquot of this Nile red stock solution was pipetted into $1 \mathrm{~mL}$ suspensions of 10 live cercariae in water for a final concentration of $0.002 \mu \mathrm{g}$ Nile $\mathrm{red} / \mathrm{mL}$ of cercariae suspension (Frandsen and Christensen 1984; Chen et al. 2009; Held and Raymond 2011). Cercariae were $\leq 2 \mathrm{~h}$ old at time of use for this procedure. Other studies have employed similar dilute concentrations for algal cell staining, and very dilute concentrations for cercariae staining, with the concentration used here similar to Frandsen and Christensen (1984). The solution was gently agitated for $30 \mathrm{sec}$ to ensure that the stain was evenly distributed throughout the suspension and then allowed to sit, unagitated, for $10 \mathrm{~min}$. One drop of formaldehyde (252549, ACS Reagent Grade, Sigma Aldrich) was then added to the suspension just prior to fluorescence microscopy to fix the cercariae and prevent their movement.

\section{Fluorescence Microscopy}

Ten cercariae of each species/type were pipetted from their $1 \mathrm{~mL}$ suspension and placed onto microscope slides. Thirty-gram cover slips were then placed over the sample. Fluorescence and white light images were taken using a Leica DM500 microscope. The excitation and emission wavelengths used in previous studies (Alonzo and Mayzaud 1999), were $530 \mathrm{~nm}$ and $560 \mathrm{~nm}$ to fluoresce neutral lipids. My images were taken at the following wavelengths: $546 \mathrm{~nm}$ excitation, $560 \mathrm{~nm}$ emission and $605 \mathrm{~nm}$ suppression filter. All images were taken at 10X magnification with the following settings: fluorescence exposure at $90 \mathrm{~ms}$, gain at 4.8X, and gamma at 1.0. Adjusting the gain setting amplifies the image signal, which results in a brighter image without increasing the exposure time. The gamma setting adjusts the contrast range and how it is applied to an image, with a gamma value of 1.0 indicating that the contrast is applied evenly (linearly) across the whole light intensity range. To establish the appropriate settings for my images, including exposure time, I took 10 images of each cercariae species/type at a range of exposures to determine a single optimal exposure time for all images. This was done so that I could 
quantitatively compare the fluorescence, and thus lipid content, across different cercariae species/types. I used ImageJ (version 1.50i) software to determine which cercariae were the most and least fluorescent, i.e. contained more or less lipids, using mean fluorescence values. I then established which exposure time would fluoresce my least fluorescent image without saturating my most fluorescent image, and chose that exposure as the final setting for all samples. In total, images were acquired for 20 cercariae from each of the 5 different species/types ( $R$. ondatrae, Echinostoma spp., Haematoloechus sp., and armatae- and strigeid-type cercariae).

\section{Lipid Fluorescence Binning Technique}

As I had images for 20 cercariae of each species/type, 100 cercariae in total were analyzed using an optical binning method in order to identify and quantify differences in the distribution patterns of neutral lipid energy reserves (i.e. triacylglyceride $=$ TAG and diacylglyceride $=\mathrm{DAG}$ ) among the parasite species. A similar binning technique was used in a previous study to determine seasonal differences in energy reserve lipids in calanoid copepods (Arts and Evans 1991). Based on the fluorescent images of each parasite, cercariae were divided into 4 body segments (see below) based on key morphological features (Fig. 2.1). An example using Echinostoma sp. cercaria illustrates how images were divided as part of this binning technique (Fig. 2.1). Cercariae from other species differed in their body shapes, from having shorter and wider body segment forms (i.e. armatae-type), to long and thin body forms (i.e. strigeid-type). However, each parasite image consistently compartmentalized into 4 discrete segments (A, B, C, D), and each segment contained the following morphological components:
A: Mouth, oral sucker, and pharynx
B: Oesophagus and cytogenous glands
C: Intestine, central sucker (acetabulum), excretory bladder, excretory duct, and reproductive rudiments

D: Tail, i.e. end of the excretory duct

The level of fluorescence was then determined for each of the four above segments. Each cercaria was visually placed into a corresponding fluorescence level bin $(0,1,2,3)$ based on specific, repeatable, criteria pertaining to the fluorescence intensity and location of the fluorescence in its image (Table 2.1). This allowed for a quantitative comparison of fluorescence, and hence, neutral lipid energy reserve levels, among the cercariae of the five different parasite species/types. 

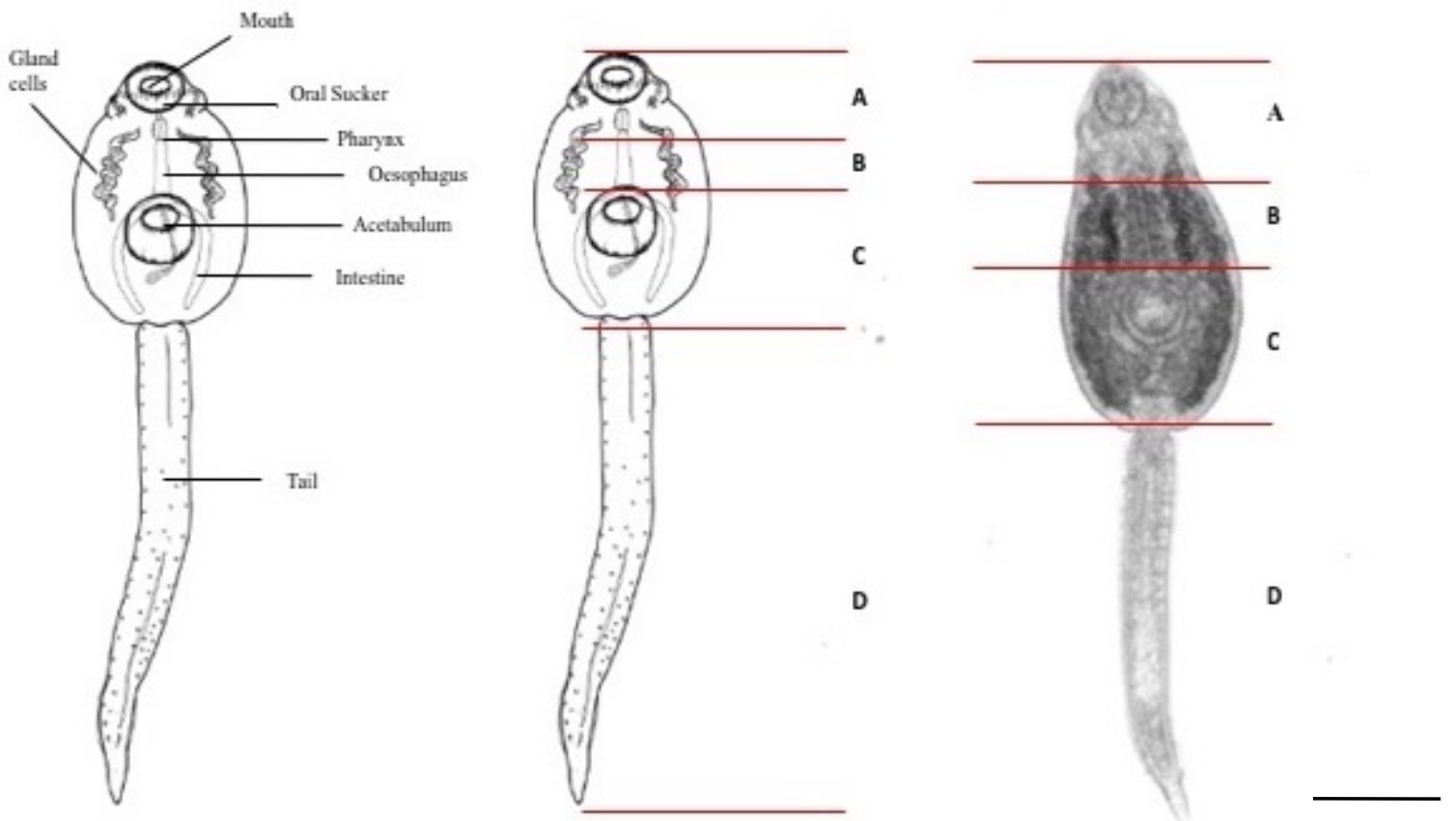

Figure 2.1. Schematic representation of Echinostoma sp. cercariae showing the body regions (3 body and one tail segments) wherein fluorescent lipids were visually observed. Image on the far right taken using light microscopy, scale-bar: $100 \mu \mathrm{m}$ (the two drawings on the left are from Georgieva et al. 2013). 


\section{Neutral Energy Reserve Lipid Concentration Curve}

A concentration curve was created to quantify the Nile red fluorescence levels (associated with neutral energy reserve lipids) in the parasite images. Pure fish oil (which is predominantly TAG) was stained with Nile red dissolved in acetone at a concentration of $0.002 \mu \mathrm{g} / \mathrm{mL}$, the same concentration as used with cercariae. The stained neutral lipid droplets were then suspended in ultrasound gel (Parker Labs, 03-02) to ensure the maintenance of spherical or ellipsoidal lipid droplets. The ultrasound gel with scattered droplets was then plated onto a microscope slide and a cover slip was placed over top. Images of the stained droplets were taken using the same microscope and settings as described above to ensure comparability to the parasite images.

The lipid droplets in the images were 2-D, therefore to estimate the volume of the droplets, I used a prolate spheroid model to approximate the shape and volume (as in Arts and Evans 1991):

$$
\text { Volume }=\frac{4}{3} \pi \times\left(\frac{A}{2}\right)^{2} \times\left(\frac{B}{2}\right)
$$

Where $A$ is the short axis (both perpendicular short axes assumed to be equal in length) and $B$ is the long axis.

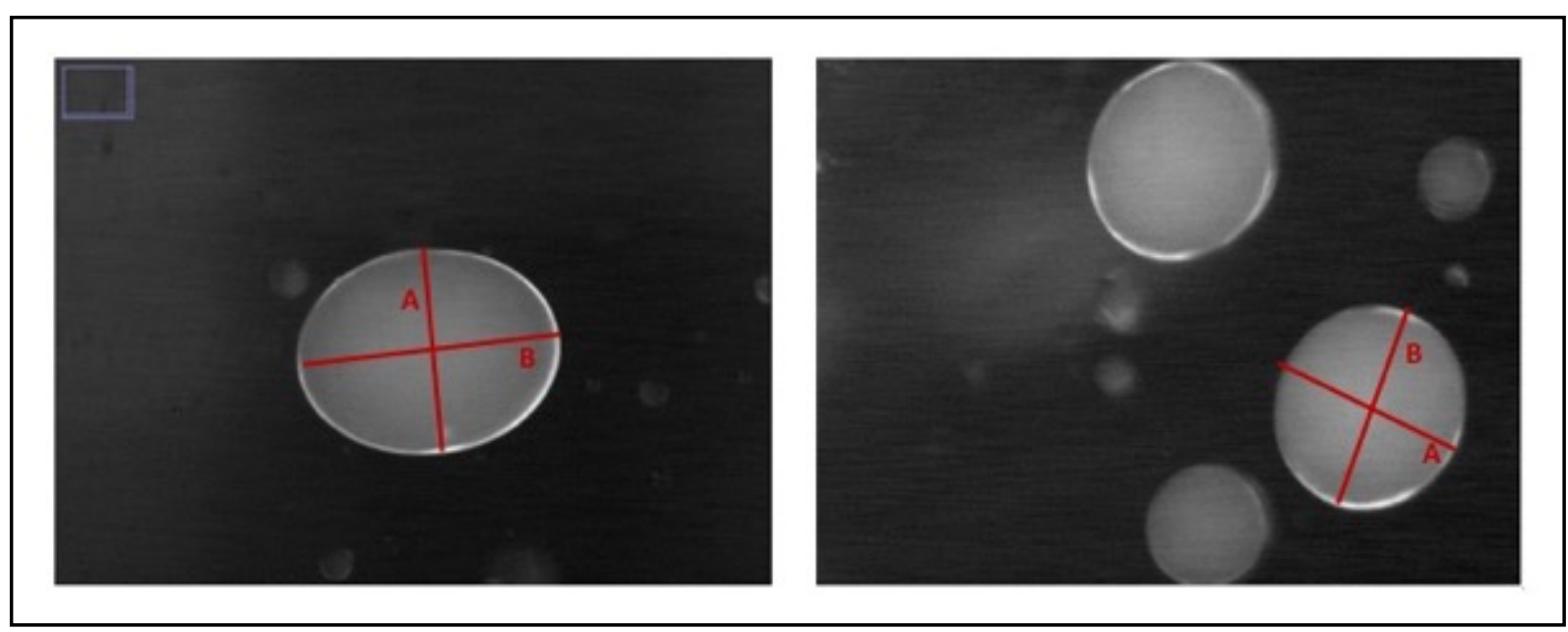

Figure 2.2. Nile red stained fish oil (neutral lipid energy reserve) droplets suspended in ultrasound gel taken using fluorescence microscopy. Short axis (A) and long axis (B) indicate measurements taken to determine the approximate volume of each droplet. 
Table 2.1: Example of binning criteria for optical quantification of neutral energy reserve lipids for cercariae of trematode parasites (Echinostoma sp.). Refer to Fig. 2.1 for an explanation of the letters (signifying body regions) B, C, and D.

\begin{tabular}{|c|c|c|c|c|}
\hline Bin & 0 & 1 & 2 & 3 \\
\hline Example & & & & \\
\hline Criteria & 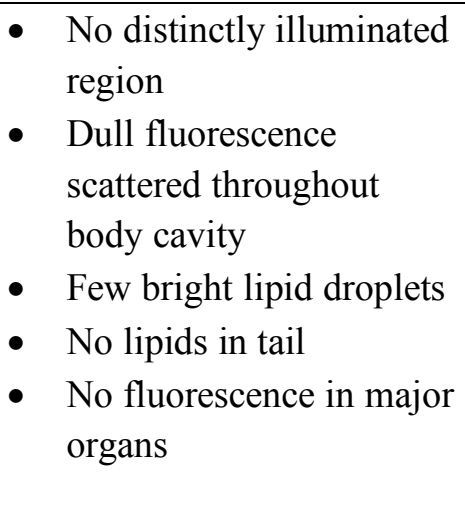 & $\begin{array}{ll}\text { - } & \text { Lipids in B \& C } \\
\text { - } & \text { Dense lipids throughout } \\
\text { esophagus \& intestine } \\
\text { - } \\
\text { Dim scattered lipids } \\
\text { throughout body } \\
\text { - Concentrated lipids in } \\
\text { lower body region } \\
\text { - Few bright droplets in tail } \\
\text { - Lipids absent from } \\
\text { acetabulum }\end{array}$ & 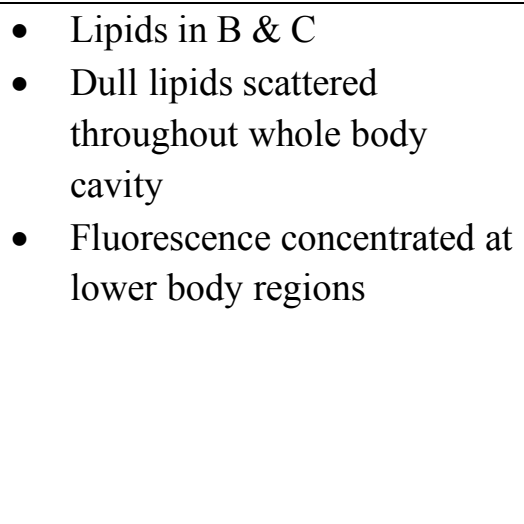 & $\begin{array}{ll}\text { - } & \text { Lipids in B, C \& D } \\
\text { - } & \text { Lipids present along } \\
\text { exterior of body cavity } \\
\text { - } & \text { Lipids scattered throughout } \\
\text { rest of body } \\
\text { - } & \text { Concentrated lipids in lower } \\
\text { half } \\
\text { - } \\
\begin{array}{l}\text { Lipids absent from } \\
\text { acetabulum }\end{array}\end{array}$ \\
\hline
\end{tabular}


The short and long axis of each lipid droplet was measured in $\mu \mathrm{m}$ (Fig. 2.2). Droplets were then defined to determine the fluorescence intensity as per the fluorescence mean, with background fluorescence subtracted from the mean. The volume of each droplet was calculated in $\mu \mathrm{m} 3$ and then converted $\mu 1$ (see equation above). This volume (if it were water) was then multiplied by the density of tripalmitin $(0.8752 \mu \mathrm{g} / \mu \mathrm{l})$, i.e. using the assumption the lipid from the fish oil pills was pure tripalmitin (C51H98O6). The fluorescence intensity (arbitrary units) of the droplet was then plotted vs the total neutral energy reserve lipid ( $\mu$ g) (Fig. 2.3). This resulted in the equation: $y=$ 9E-06x - 0.0124, $\mathrm{R}^{2}=0.7523$ (Fig. 2.3).

\section{Fatty Acid Analysis of Ribeiroia ondatrae Cercariae}

Live $R$. ondatrae cercariae were collected from $H$. trivolvis snails obtained from California field sites (see Field Collection and Animal Care above) over a period of several weeks. In total, $\sim 30,000 R$. ondatrae cercariae were collected and pipetted into $1.5 \mathrm{~mL}$ microcentrifuge tubes that were subsequently kept frozen at $-20^{\circ} \mathrm{C}$ for 1 month and then transferred to $-80^{\circ} \mathrm{C}$ until analysis. Prior to filtering, cercariae were thawed and poured through a $5.0 \mu \mathrm{m}$ silver metal membrane filter (Flotonics Division Selas Corporation of America, FM-47) inside a sealed filtration apparatus attached to a pressure-controlled vacuum pump (1VAF-10-M100X, GAST). The Silver Metal Membrane filter was used due to its durability and resistance to degradation when exposed to flowing fluids and, importantly, because parasites can be gently scraped off the filters, using a soft-pliable solvent-cleaned silicone spatula, without the complication of glass fibers (as is the case with $\mathrm{GF} / \mathrm{C}$ and $\mathrm{GF} / \mathrm{F}$ filters) which can also be removed during the scraping process. The cercariae were filtered with the pressure maintained at 3.0 Barr to ensure that they would not burst. The filter was scraped multiple times during and after filtration to ensure that the cercariae were not forced into the filter during filtration. The cercarial mass was weighed using a Mettler Toledo microbalance (Mettler Toledo \#XP6) with $1 \mu \mathrm{g}$ precision. The wet weight was determined to be $19.839 \mathrm{mg}$.

\section{Lipid Extraction}

The sample was homogenized using a Polytron PCU-2-110 homogenizer (Brinkmann Instruments, Rexdale, ON, Canada) and the lipids were extracted with $2 \mathrm{~mL}$ of a 2:1 mixture of chloroform:methanol (v/v \%) following Folch et al. (1957). Chloroform-extracted water was then added to bring the ratio of chloroform:methanol:water to 8:4:3. A known amount of 
tricosanoic acid standard (23:0 methyl ester, Nu-Chek Prep. Inc, N-23-M) was added to the sample. This internal standard was selected based on previous (Desvilettes et al. 1997; Boissonnot et al. 2016) lipid profiles of freshwater zooplankton which generally lack 23:0, and are comparable to cercariae in terms of their food sources and environment. After the addition of $0.8 \mathrm{~mL}$ of $0.88 \%$ aqueous $\mathrm{KCl}(\mathrm{w} / \mathrm{v})$, the sample was homogenized using a vortex and centrifuged at $2000 \mathrm{rpm}$ for $5 \mathrm{~min}$ to separate the phases. The bottom organic layer containing chloroform and lipids was recovered using a double pipetting technique by placing a $2 \mathrm{~mL}$ Pasteur pipette inside a $1 \mathrm{~mL}$ pipette (both pipettes were solvent-washed) to remove the organic layer without disturbing the top aqueous layer (Parrish 1999). More chloroform was added, and the sample was re-extracted (a total of $3 \mathrm{X}$ ). The extract from all three extractions was pooled into a solvent-washed glass round bottom test tube and evaporated to dryness under a continuous $\mathrm{N}_{2}$ stream. The sample was reconstituted by adding $2 \mathrm{~mL}$ of hexanes and ensuring that the sides of the tube were washed down during the addition of the hexanes. Gravimetric analysis was done by first recording the weight of 2 empty Ultra-Clean Pressed Tin Capsules (D1029, Isomass Scientific Inc., Calgary CA) and placing $100 \mu \mathrm{L}$ aliquots of the hexane solution into each tin capsule. The solvents were then evaporated at room temperature, and re-weighed to provide an estimate of total lipid.

Fatty acid methyl esters (FAME) were produced using the acid-catalyzed transesterification method, using $2 \mathrm{~mL}$ of $\mathrm{H}_{2} \mathrm{SO}_{4}$ in methanol (1\%, v/v) (Christie 1989). The samples were sealed in the tube with $\mathrm{N}_{2}$ gas and heated at $90^{\circ} \mathrm{C}$ for $90 \mathrm{~min}$. After the sample cooled for $5 \mathrm{~min}, 1.5 \mathrm{~mL}$ of Milli-Q water and $4 \mathrm{~mL}$ of hexanes were added to stop the reaction. The solution was vortexed and centrifuged at $2000 \mathrm{RPM}$ for $3 \mathrm{~min}$ at $4^{\circ} \mathrm{C}$. The top layer (hexanes) was decanted with a short Pasteur pipette, and $4 \mathrm{~mL}$ of hexanes were added to the remaining bottom layer (water); this process was repeated $3 \mathrm{X}$. The final sample was evaporated to dryness under an $\mathrm{N}_{2}$ stream, reconstituted with $100 \mu \mathrm{L}$ of hexanes and transferred using a Pasteur pipette into a labelled GC vial with a straight $400 \mu \mathrm{L}$ insert. This process was repeated, adding another $100 \mu \mathrm{L}$ of hexanes to the GC vial. The GC vial was then evaporated to dryness and $100 \mu \mathrm{L}$ of hexane were added as the final sample volume. The sample was stored in at $-85^{\circ} \mathrm{C}$ until analysis.

The sample was analyzed using a Gas Chromatography (GC; Shimadzu GC-2010 plus) equipped with a flame ionization detector and helium used as the carrier gas. The sample was injected splitless with an initial column temperature of $60^{\circ} \mathrm{C}$. The temperature was then increased 
to $250^{\circ} \mathrm{C}$ over $37 \mathrm{~min}$ and held at $250^{\circ} \mathrm{C}$ for $23 \mathrm{~min}$. FAME were identified based on their retention times. The FAME were quantified by comparing their responses (peak areas) to a standard concentration curve prepared using a standard FAME mix (GLC 68E, Nu-Check Prep, Inc.) and standard QA/QC was performed in relation to the internal standard (23:0, $1 \mu \mathrm{g} / \mathrm{sample})$.

\section{Results}

Lipid energy reserve levels determined via the optical binning technique (Fig. 2.5) significantly correlated to the fluorescence intensity, whereby the total lipids were determined using the ultrasound gel concentration curve (Fig. 2.6). Binning value and fluorescence intensity were significantly correlated for both the bin assignment and the species respectively $(\mathrm{df}=3, \mathrm{~F}=$ $13.78, \mathrm{P}=0.000 ; \mathrm{df}=4, \mathrm{~F}=6.078, \mathrm{P}=0.014)$. The concentration curve allowed for the estimation of individual cercaria lipid droplets (Fig. 2.6). Armatae-type cercariae were most often placed in the lower fluorescence level bin, which suggests that armatae-type cercariae contained the least neutral energy reserve lipids/animal $(\mu \mathrm{g})$ with a mean value of $0.047 \mu \mathrm{g}$ of neutral energy reserve lipids/animal (Figs. 2.5 \& 2.6). Echinostoma sp., R. ondatrae and strigeidtype cercariae contained higher mean lipid energy reserve levels compared to armatae-type cercariae (Echinostoma sp.: $0.056 \mu \mathrm{g}$; R. ondatrae: $0.092 \mu \mathrm{g}$; strigeid-type: $0.045 \mu \mathrm{g}$ ) (Fig. 2.6) and were also binned to contain higher fluorescence levels (Fig. 2.5). Haematoloechus sp. cercariae had the highest mean value with $0.24 \mu \mathrm{g}$ of lipid energy reserves/animal and was most frequently assigned to the highest bin value (Figs. $2.5 \& 2.6$ ). 


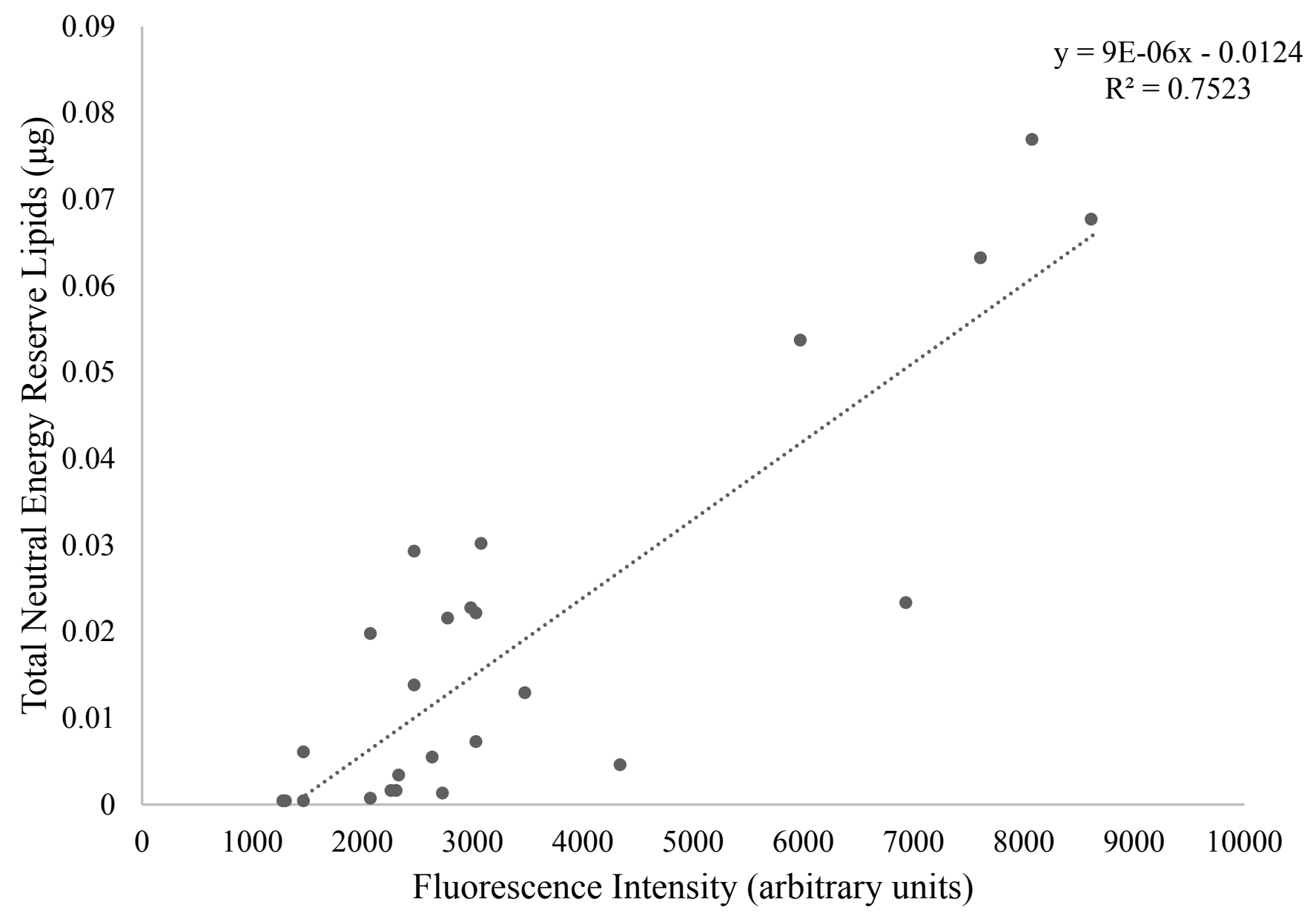

Figure 2.3. Concentration curve of Nile red stained fish oil (neutral energy reserve lipid) suspended in ultrasound gel, using fluorescence microscopy. $\left(y=9 \mathrm{E}-06 \mathrm{x}-0.0124, \mathbf{R}^{2}=0.75\right)$ 
A FA profile for $R$. ondatrae cercariae was achieved from GC analysis on an aggregate of $\sim 30,000$ cercariae. Gravimetric analysis determined that the total lipid extracted from the aggregate $R$. ondatrae cercariae sample $(11 \mathrm{mg})$ was $8.64 \%$ of the dry weight, allowing for an estimation of $0.0317 \mu \mathrm{g}$ of total lipid/individual $R$. ondatrae cercaria. The cercariae aggregate contained $449.98 \mu \mathrm{g}$ of identified FA, which provides the estimation of $15.0 \mathrm{ng}$ of identified FA/cercariae. Individual $R$. ondatrae cercariae contained high amounts (hereafter reported as $\mathrm{ng} /$ cercariae DW) and proportions of 16:0 (2.8: 19.02\%); 18:0 (2.0; 13.59\%); 18:1n-9c (0.92; $6.15 \%)$ and 20:3n-3 (1.0; 6.80\%) (Table 2.2). Cercariae also contained all five EFA: LNA at $0.68 \mathrm{ng} /$ cercariae DW; ALA at $1.8 \mathrm{ng} /$ cercariae DW; ARA at $0.25 \mathrm{ng} /$ cercariae DW; EPA at $0.791 \mathrm{ng} /$ cercariae DW; and DHA at $0.1 \mathrm{ng} /$ cercariae DW) (Table 2.2). FA sums indicated that within $R$. ondatrae cercariae SFA constituted the highest proportion and mass fraction of FAs (6.56; 43.77\%), followed closely by PUFA (5.10; $34.0 \%)$, and lastly, MUFA $(3.33 ; 22.23 \%)$. Finally, $R$. ondatrae cercariae contained greater proportions of n-3 FA $(3.67 ; 24.46 \%)$ compared to n-6 FA $(1.43 ; 9.55 \%)$. 


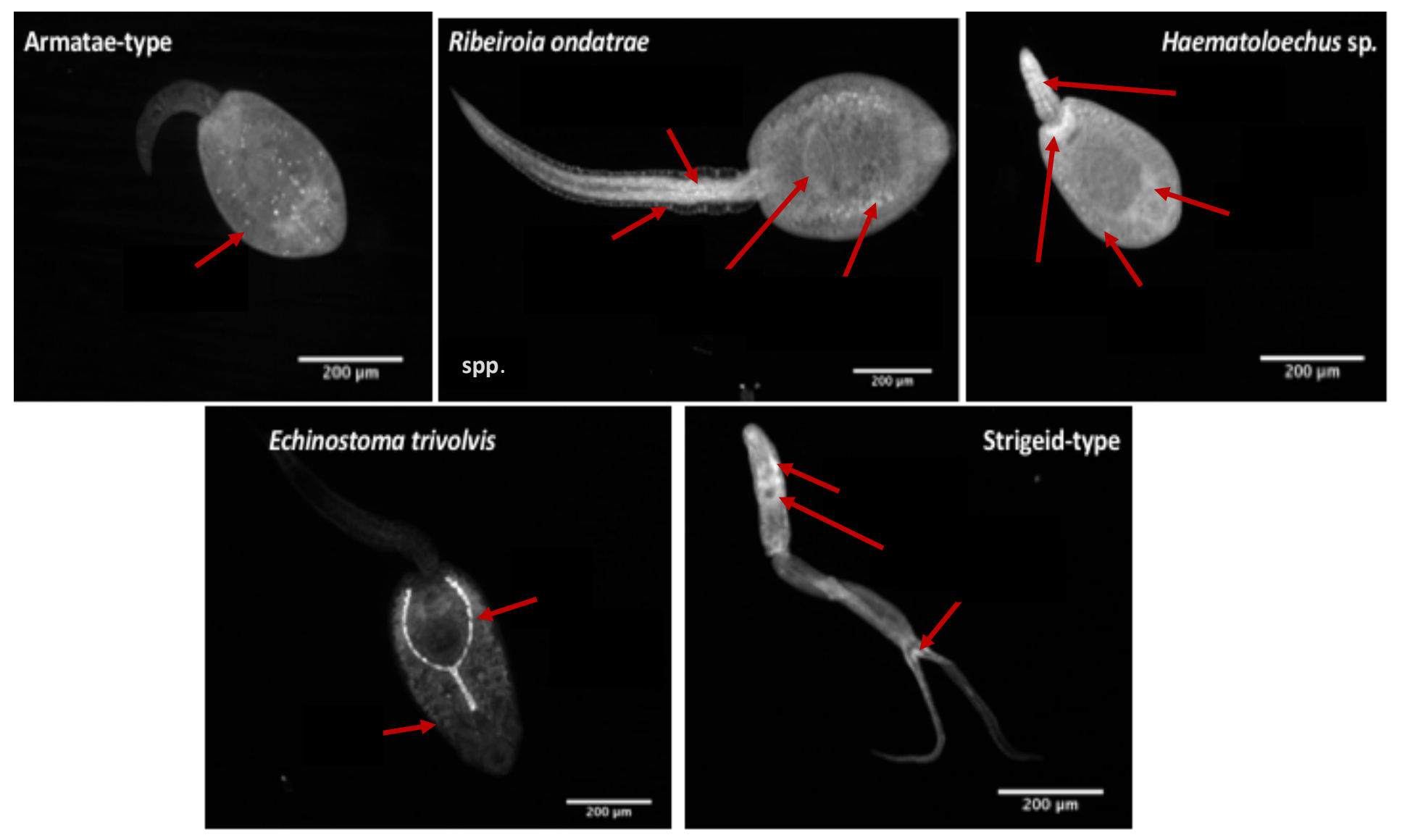

Figure 2.4. Nile red stained cercariae of five different species/types. These images and other replicates used to determine relative fluorescence and neutral energy reserve levels among species. Scale bar and cercaria identification in : gure Atbreviations used: $A=$ acetabulum; AD $=$ acetabular ducts; $\mathrm{ET}=$ excretory tubule; $\mathrm{EV}=$ sxcrı $\curvearrowright r y$ vesicle; $\mathrm{FC}=$ flame cells; $\mathrm{IT}=$ intestine; $\mathrm{PG}=$ penetration glands. 

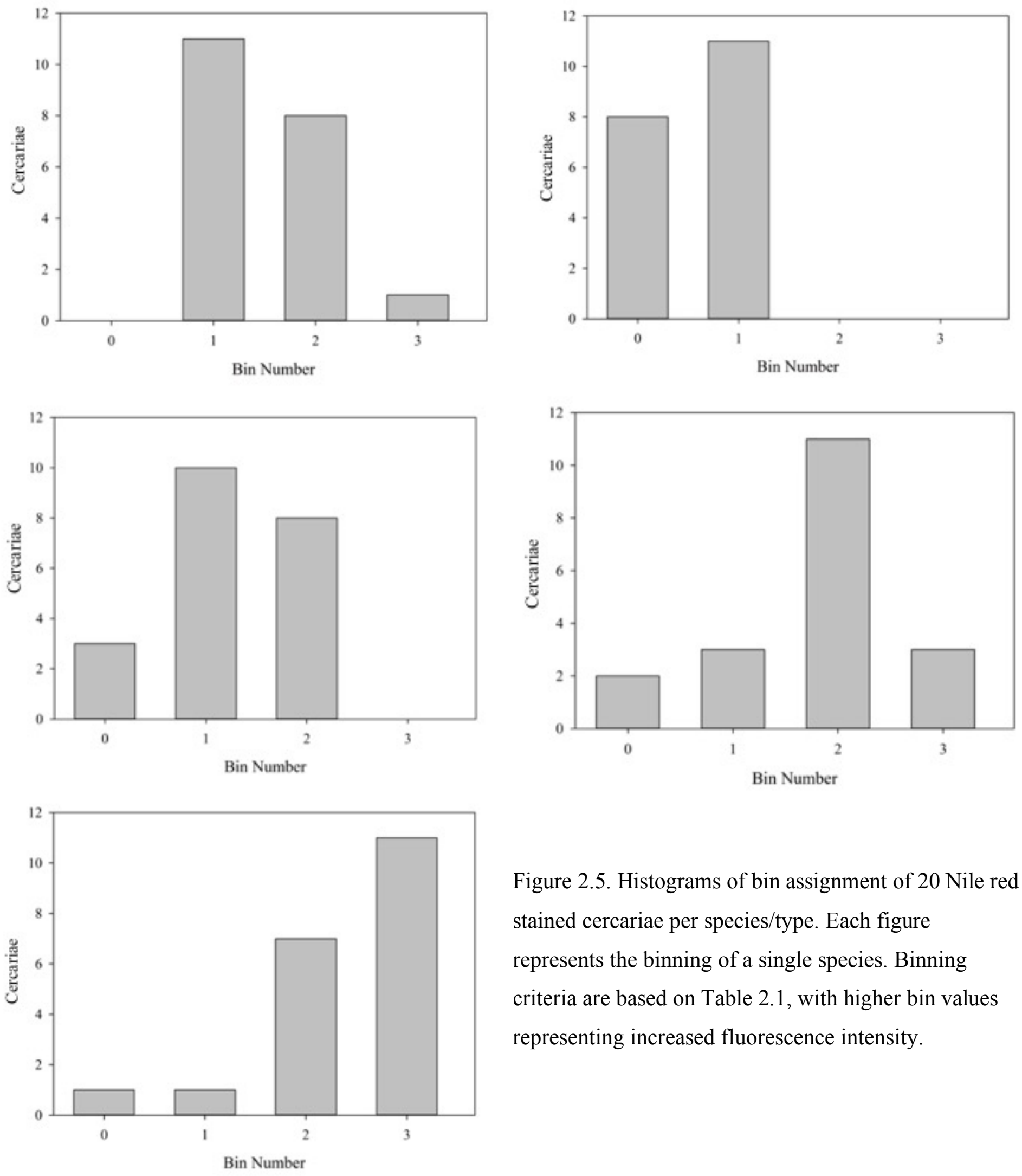

Figure 2.5. Histograms of bin assignment of 20 Nile red stained cercariae per species/type. Each figure represents the binning of a single species. Binning criteria are based on Table 2.1, with higher bin values representing increased fluorescence intensity. 


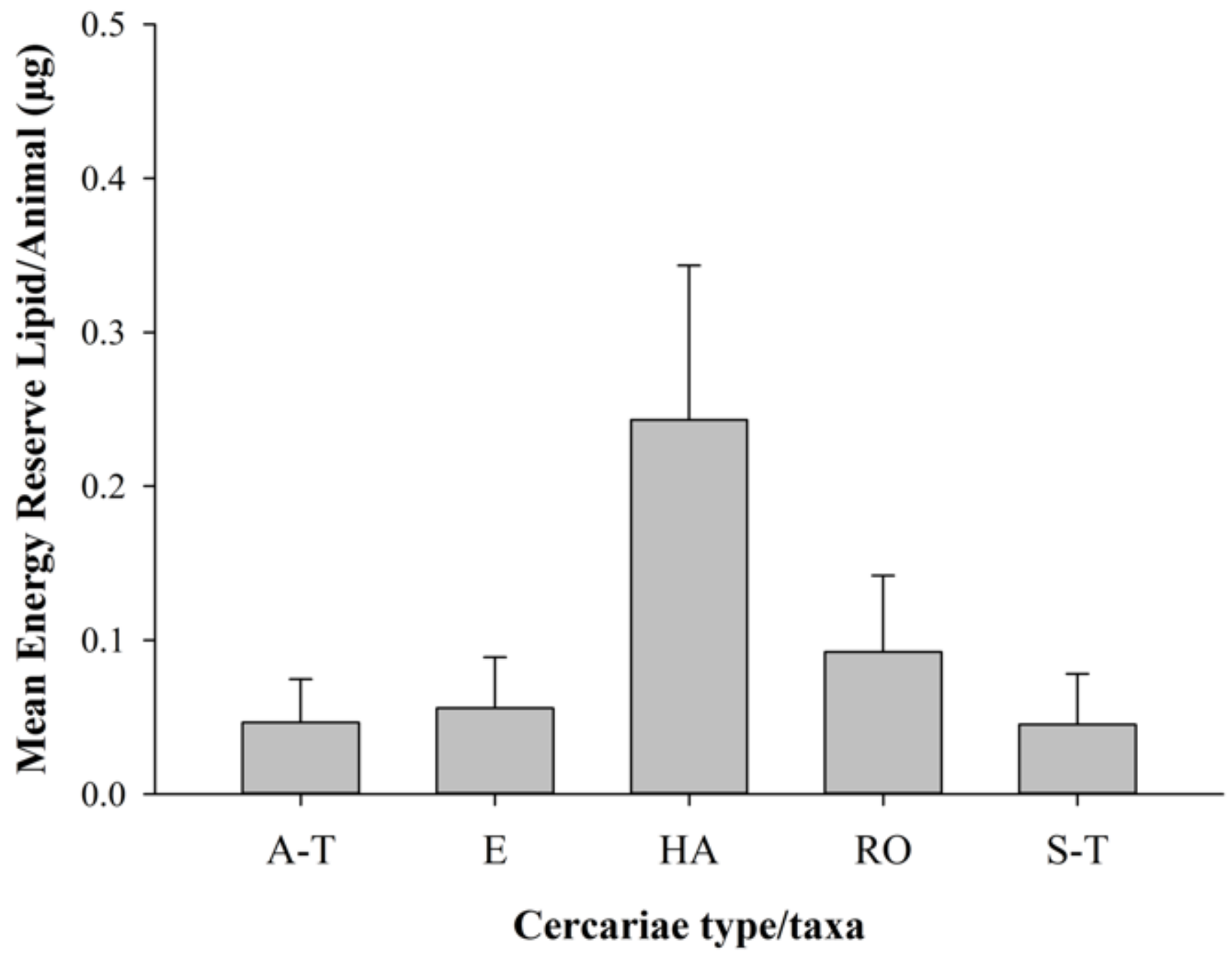

Figure 2.6. Estimated mean energy reserve lipids $(\mu \mathrm{g})$ in Nile red-stained cercariae grouped by taxa/type (A-T: armatae-type, E: Echinostoma sp.,2 HA: Haematoloechus sp., RO: Ribeiroia ondatrae, S-T: strigeid-type) as determined using the fluorescence intensity of triacylglycerol droplets concentration curve (Fig. 2.3). Error bars: +/1 SD. 
Table 2.2: Fatty acid composition of the Ribeiroia ondatrae cercariae sample on a DW, WW and proportional ( $\%$ of total identified FA) basis. These results stem from a single FA analysis representing an aggregate of $\sim 30,000$ cercariae.

\begin{tabular}{|c|c|c|c|c|}
\hline Fatty acids & Mass Fraction $(\mu \mathrm{g} / \mathrm{mg}) \mathrm{DW}$ & Mass Fraction $(\mu \mathrm{g} / \mathrm{mg}) \mathrm{WW}$ & Proportional (\% of total FA) & Mass Fraction (ng/cercariae) DW \\
\hline $14: 0$ & 0.77 & 0.43 & 1.88 & 0.28 \\
\hline $14: 1 n-5$ & 0.31 & 0.17 & 0.76 & 0.11 \\
\hline $15: 0 \mathrm{i}$ & - & - & - & - \\
\hline $15: 0 a$ & - & - & - & - \\
\hline $15: 0$ & 0.80 & 0.44 & 1.95 & 0.29 \\
\hline $15: 1 n-5$ & 0.02 & 0.01 & 0.06 & 0.00 \\
\hline $16: 0$ & 7.78 & 4.31 & 19.02 & 2.85 \\
\hline $16: 1 n-9$ & - & - & - & - \\
\hline $16: 1 n-7 c$ & 1.09 & 0.60 & 2.67 & 0.40 \\
\hline $16: 1 n-7 t$ & 0.98 & 0.54 & 2.39 & 0.36 \\
\hline 17:0 & 0.69 & 0.38 & 1.68 & 0.25 \\
\hline $17: 1 n-7$ & 0.03 & 0.02 & 0.07 & 0.01 \\
\hline 18:0 & 5.56 & 3.08 & 13.59 & 2.04 \\
\hline $18: 1 n-9 c$ & 2.51 & 1.39 & 6.15 & 0.92 \\
\hline $18: 1 n-9 t$ & 0.05 & 0.03 & 0.12 & 0.02 \\
\hline $18: 1 \mathrm{n}-12 \mathrm{c}$ & - & - & - & - \\
\hline $18: 1 n-7 c$ & 1.56 & 0.86 & 3.80 & 0.57 \\
\hline $18: 1 n-7 t$ & 0.63 & 0.35 & 1.53 & 0.23 \\
\hline 19:0 & 0.17 & 0.09 & 0.41 & 0.06 \\
\hline 19:1n-12 & 0.10 & 0.06 & 0.25 & 0.04 \\
\hline $18: 2 n-6 c$ (LNA) & 1.86 & 1.03 & 4.55 & 0.68 \\
\hline 20:0 & 0.48 & 0.26 & 1.16 & 0.17 \\
\hline $18: 3 n-6$ & - & - & - & - \\
\hline $20: 1 n-15$ & 0.02 & 0.01 & 0.04 & 0.01 \\
\hline $20: 1 n-12$ & - & - & - & - \\
\hline 20:1n-9 & 0.54 & 0.30 & 1.32 & 0.20 \\
\hline $18: 3 n-3$ (ALA) & 5.02 & 2.78 & 12.26 & 1.84 \\
\hline 19:OD & - & - & - & - \\
\hline $20: 1 n-11$ & - & - & - & - \\
\hline $20: 2 n-6$ & 0.67 & 0.37 & 1.63 & 0.24 \\
\hline $22: 3 n-3$ & 1.12 & 0.62 & 2.74 & 0.41 \\
\hline $22: 0$ & 0.55 & 0.31 & 1.35 & 0.20 \\
\hline $20: 3 n-6$ & 0.60 & 0.33 & 1.46 & 0.22 \\
\hline $22: 1 n-11$ & - & - & - & - \\
\hline $22: 1 n-9$ & 0.08 & 0.04 & 0.19 & 0.03 \\
\hline $20: 3 n-3$ & 2.78 & 1.54 & 6.80 & 1.02 \\
\hline $20: 4 n-6$ (ARA) & 0.68 & 0.38 & 1.66 & 0.25 \\
\hline $22: 2 n-6$ & 0.05 & 0.03 & 0.12 & 0.02 \\
\hline $24: 0$ & 1.12 & 0.62 & 2.74 & 0.41 \\
\hline $20: 5 n-3$ (EPA) & 2.16 & 1.20 & 5.27 & 0.79 \\
\hline $24: 1 n-9$ & 0.05 & 0.03 & 0.12 & 0.02 \\
\hline $22: 4 n-6$ & 0.05 & 0.03 & 0.13 & 0.02 \\
\hline $22: 5 n-6$ & - & - & - & - \\
\hline $22: 5 n-3$ & 0.01 & 0.01 & 0.03 & 0.00 \\
\hline $22: 6 n-3(\mathrm{DHA})$ & 0.04 & 0.02 & 0.09 & 0.01 \\
\hline $18: 4 n-3$ & - & - & - & - \\
\hline \multicolumn{5}{|l|}{$\Sigma$} \\
\hline Sum SFA & 17.91 & 9.93 & 43.77 & 6.57 \\
\hline Sum MUFA & 9.09 & 5.04 & 22.23 & 3.33 \\
\hline Sum PUFA & 13.91 & 7.71 & 34.00 & 5.10 \\
\hline Sum MUFA $\geq 18$ & 6.66 & 3.69 & 16.27 & 2.44 \\
\hline Sum MUFA>18 & 1.91 & 1.06 & 4.67 & 0.70 \\
\hline Sum C18 PUFA & 6.88 & 3.81 & 16.82 & 2.52 \\
\hline Sum C20 PUFA & 6.88 & 3.82 & 16.82 & 2.52 \\
\hline Sum C22 PUFA & 0.15 & 0.08 & 0.36 & 0.05 \\
\hline Sum EPA/DHA & 2.19 & 1.22 & 5.36 & 0.80 \\
\hline Sum n-6 & 3.90 & 2.17 & 9.55 & 1.43 \\
\hline Sum n-3 & 10.00 & 5.55 & 24.46 & 3.67 \\
\hline Sum Odd chain & 4.40 & 2.44 & 10.76 & 1.61 \\
\hline$n-3 / n-6$ & 2.56 & 2.56 & 2.56 & 0.09 \\
\hline Total & 40.91 & 22.68 & 100.00 & 15.00 \\
\hline
\end{tabular}

-, not detected. LNA: linolenic acid; ALA: alpha-linolenic acid; ARA: arachidonic acid; EPA: eicosapentaenoic acid; DHA: docosahexaenoic acid 


\section{Discussion}

I demonstrate the lipid-rich nature of trematode parasite cercariae, and their consequent ability to contribute to nutrient flow in aquatic ecosystems, through the development of an opticalbinning technique for histochemical analysis as well as the calculation of EPA and DHA in $R$. ondatrae cercariae through FA analysis (via gas chromatography, i.e. GC). Distinct amounts of neutral energy reserve lipids were detected in each of the five cercariae species/types investigated ( $R$. ondatrae, Haematoloechus sp., Echinostoma sp., strigeid-type and armatae-type) through Nile red staining and quantitative analysis using the optical-binning technique. Visual differences in the localization and amount of lipid deposits in each cercariae species/type revealed type-specific variation in lipid quantity. GC analysis on the aggregate sample of $\sim 30,000 R$. ondatrae cercariae revealed that individual cercariae contain $\sim 0.288 \mu \mathrm{g}$ of total lipids/cercariae and $\sim 15.0 \mathrm{ng}$ total identified FA each (Table 2.2). Specifically, $R$. ondatrae cercariae were found to contain significant quantities of SFA, including MUFA (18:1n-9), n-3 PUFA (20:3n-3, 20:5n-3, ALA [18:3n-3], ARA [20:4n-3], EPA [20:5n-3], DHA [22:6n-3]), and n-6 PUFA (LNA [18:2n-6]) (Table 2.2).

The optical-binning technique, developed to rapidly categorize the amount of visible lipid droplets in individual cercariae, reflected the extreme variation in lipid energy reserve content likely driven, in part, by cercariae morphology. The binning technique significantly correlated to the quantitative estimations of cercariae lipid energy reserve content (using the fluorescence concentration curve). For instance, Haematoloechus sp. cercariae were most often assigned to the highest bin assignment and were also estimated to contain the highest amount of lipid energy reserves, followed by strigeid-type cercariae and $R$. ondatrae (Fig. 2.5). Echinostoma $s p$. cercariae were frequently assigned to lower bin assignments, followed by armatae-type cercariae, and both were estimated to contain lower lipid level via the concentration curve (Fig. 2.5, Fig. 2.6). Notably, although these results were obtained with the same cercariae images, this technique demonstrates the comparability of these techniques and their reliable replication. Additionally, a previous study using the optical-binning technique for quantitative analysis of seasonal lipid differences among copepods (Arts and Evans 1991) showed similar variation in total TAG (albeit seasonally within one species). My study is the first to develop and use an optical-binning technique to quantitatively estimate cercariae neutral energy reserve lipids, and the development of this method for use on individual infectious stages will be beneficial for 
future work in this area. Notably, other lipid analysis methods, such as FA analysis, require a large number of cercariae for a single sample $(\sim 15 \mathrm{mg}$ dry weight $=\sim 30,000$ cercariae per sample for $R$. ondatrae), as well as expensive equipment, consumables, and software. The ability to utilize a relatively simple method to quantitatively estimate parasite lipid levels is thus valuable to better understand individual and taxon-level variability in the composition of trematode cercariae, as well as other small aquatic invertebrates.

The Nile red staining technique identified lipid-rich regions in cercariae; and was a useful technique to estimate the neutral lipid stores in cercariae. The ultrasound gel concentration curve method estimated $0.092 \mu \mathrm{g}$ of mean energy reserve lipid/cercariae for $R$. ondatrae. Although not directly comparable, gravimetric analysis for $R$. ondatrae cercariae indicated $0.317 \mu \mathrm{g}$ of total lipid/cercariae, and fatty acid analysis indicated $15.0 \mathrm{ng}$ of FA/cercariae (Fig. 2.6, Table 2.2). In comparing the Nile red concentration curve lipid estimates to both the total lipids and FA per cercariae, the neutral lipid estimation made by the curve was lower than the total lipids (which includes sterols and phospholipids) and was higher than the FA estimates, which was expected. Total lipids determined gravimetrically detects all lipid types, including polar lipids, such as sterols and phospholipids. Neutral and polar lipids have been found to be simultaneously detected at any wavelength, and therefore additional polar species can contribute $10-20 \%$ interference when quantifying neutral lipids using fluorescence techniques (Alonzo and Mayzaud 1999). Fluorometric and image-based quantification of both polar and neutral lipids in small organisms using Nile red have been proposed (Hentschel 1998), and provided robust results when compared to gravimetric methods (Cooksey et al. 1987; Carman 1991; Priscu et al. 1990), however the interference of polar lipids was suggested as a possible source of error when quantifying neutral lipids (Kimura et al. 2004). Therefore, my neutral lipid estimations using the curve, may be slight overestimations of the total neutral energy reserve lipids in cercariae, due to potential fluorescence from polar lipid species. Previous studies have shown that diglycerides and monoglycerides are the principal lipids in $S$. mansoni cercariae ( $73 \%$ combined), and are an abundant lipid fraction in Z. lunata cercariae (Furlong et al. 1995; Marsit et al. 2000b). These high fractions of polar lipids may have resulted in some interference of fluorescence. The estimations for $R$. ondatrae cercariae were relatively close for both the gravimetric method and the concentration curve (calibration method), which suggests that the binning technique may be useful to estimate lipid poor and rich cercariae (Fig. 2.5, Fig 2.6). Other studies have used Nile 
red to stain cercariae (Moore and Halton 1975; Diconza and Basch 1976; Furlong et al. 1972; Xu et al. 1994; Marsit et al. 2000a), but this is the first to quantitatively estimate fluorescence values based on lipid composition.

The gross external and internal morphology of cercariae varies widely based on type, and as such, the localization of internal lipids was also highly variable (Fig. 2.4). Previous work using lipid dyes (i.e. Oil Red O [ORO] and Nile Blue Sulphate) in cercariae found similar variation in the amount and localization of lipid deposits (Moore and Halton 1975; Furlong et al. 1995; Ginetskinskaya 1988; Fried et al. 1998b; Marsit et al. 2000a,b). Differences ranged from very little lipid detection in the bodies of Z. lunata and Notocotylus sp. cercariae, to the presence of defined droplets in undefined regions of the body of other cercariae (i.e. Opisthoglyphe ranae) (Ginetskinskaya 1988; Fried et al. 1998b). I observed a similar pattern in the armatae-type cercariae, which consistently exhibited scattered but discrete lipid droplets, with some appearing to be localized to the flame cells, and others appearing in undefined body regions (Fig. 2.4).

The lack of extensive histochemical lipid analysis across a broad range of cercariae renders it difficult to accurately and consistently note where lipid droplets commonly occur in various cercariae types. The most consistent findings have been reported from investigations of lipid droplets in echinostomatid cercariae (e.g., Cercariae helvetica, Echinostoma caproni and Echinostoma sp.), where lipids have reliably been found in the excretory system (Fried and Moorone 1970; Von Brand 1973; Fried and Pucci 1976; Fried et al. 1998b). Marsit et al. (2000a) detected the presence of (ORO)-positive droplets in E. caproni cercariae in close proximity to the excretory tubules and concretions. My results support these previous results as I found high concentrations of Nile red staining in the intestinal and excretory vesicle region of Echinostoma $s p$. cercariae (Fig. 2.4). A previous study also detected localization of lipid droplets in the excretory system in Ribeiroia sp. (Fried et al. 1998b), and similar results were found here with $R$. ondatrae, where lipids were concentrated in the excretory tubules (Fig. 2.4). Haematoloechus $s p$. and strigeid-type cercariae exhibited similar patterns in lipid localization, with the most detectable TAG levels present in the excretory tubules, excretory vesicles, flame cells, acetabular ducts, and acetabulum (Fig. 2.4).

Although lipid localization varied among cercaria species/type in my study, lipids were repeatedly concentrated in structures supporting secretion and excretion. The distinctive nature of the excretory system in cercariae is of particular importance with respect to their identification 
(i.e. number and arrangement of flame cells and tubules, shape of bladder and position of pores) (Faust 1919; Hussey 1943). Although some cercariae types lack certain excretory organs, the excretory system typically includes the acetabulum, acetabular ducts, penetration glands, and flame cells, as well as excretory vesicles and tubules (Hussey 1943). The reasons for the specific localization of lipids within cercariae are mainly speculative; however, their presence in the excretory system has been associated with excretory wastes that result from the end products of carbohydrate metabolism (Faust 1919; Von Brand 1973; Gupta et al. 1974; Schariter et al. 2002). As acetyl-CoA is converted to fatty acids via lipogenesis, lipid levels can increase in the intermediate stage in the metabolism of simple sugars (Menendez and Lupu 2007).

Aside from the excretion of waste, one of the most important functions of the excretory system is the secretion of substances that promote the successful penetration of the free-living cercariae into the tegument of their next host (Faust 1919; Hussey 1943; Zd'árská 1979; Haas et al. 1997). These secretions occur in the presence of a suitable host through the detection of hosttegument lipids, or when cercariae undergo physical stress, such as desiccation or the pressure imposed by adding a coverslip to a slide preparation (Haas 1997). The properties of secretory substances released from free-living cercariae range from adherence to the host skin, disintegration of tissues, tegument transformation, and immune evasion (Zd'árská 1979). Secretions from the penetration glands in cercariae from the trematode orders Echinostomida (Zd'árská 1968, 1971) and Monostomata (Zd'árská 1970) were found to eventually travel to the cercarial tegument when under stress, where they serve to perform protective functions against host immune responses and desiccation (Zd'árská 1979). Cercarial tegument has also been identified to be particularly rich in glycogen-like material, termed the glycocalyx, which has immunomodulating properties to protect cercariae from the host's immune system postpenetration (Xu et al. 1994). Identifying high concentrations of lipids in the secretory organs of cercariae suggests that these may function to aid in the process of host penetration, as well as contribute to a reduction in the hosts' response to invasion (Salafsky et al. 1984; Fusco et al. 1986; Salafsky and Fusco 1987).

Not only did the different cercariae species/types differ in their lipid localization, they also differed, individually, in the total amount of lipid energy reserves detected (Fig. 2.5). The latter observation matches what is seen in other aquatic invertebrates collected at a single location and/or at different times and which typically exhibit a high degree of individual variability in 
distribution and total amounts of lipid stores within their bodies (Arts and Evans 1991). The differences detected among cercariae here may have been driven by a range of factors that include, but are not limited to, body length, mass, and metabolic activity, as well as the diet and lipid composition of their host snail (Båmstedt 1988, Arts and Evans 1991). Larger cercariae, such as Z. lunata, have been reported to contain higher lipid levels than smaller cercariae (Marsit et al. 2000b). However, I found Haematoloechus sp. cercariae to have the highest lipid levels based on the concentration curve estimates, and yet this species had one of the smallest cercariae investigated here (Fig. 2.5). In contrast, R. ondatrae and Echinostoma sp., which have relatively larger cercariae, had lower lipid levels despite their size (Fig. 2.5).

Cercaria activity serves as a trade-off against cercaria longevity (Lawson and Wilson 1980). Cercariae that are continuously swimming and invested in swimming mechanisms have a much shorter survival time (Anderson and Whitfield 1975). Glycogen decreases over the cercariae lifespan; however, lipid droplets have previously not been found to change dramatically in aged or new cercariae (Fried et al. 1998c). In comparing the differences in lipid composition of cercariae taxa in my results, some interesting patterns emerged. Based on the type of motility used, cercariae can be divided into two groups: 1) attached cercariae with reduced tails, which typically crawl along the substrate and 2) swimming cercariae, which are further divided into continuous and intermittent swimmers (Haas 1994). Haematoloechus sp. cercariae is a xiphidiocercaria of the suborder Plagiorchiida (Tkach et al. 2000), which are characterized as intermittent active swimmers who also spend a major part of their lifetime crawling along the bottom (Schell 1985). Potentially this type of swimming conserved lipid stores prior to quantification. Interestingly, armatae-type cercariae contained the lowest lipid levels and are also classified as a xiphidiocercaria, therefore it may also be a combination of host seeking behaviour, as well as temperature and light (Sukhdeo and Sukhdeo 2004; Zimmer et al. 2009), which may have contributed to the reduction in lipid levels. Continually swimming cercariae, who constantly seek hosts upon emergence (i.e. R. ondatrae and Echinostoma spp. cercariae) exhibited lower lipid levels (Tolstenkov et al. 2012a). Echinostoma cercariae are characterized as undergoing continuous swimming and possess a well-developed tail for swimming compared to xiphidiocercariae (Tolstenkov et al. 2012b). Finally, strigeid-type cercariae are less active swimmers and also assume a floating position (Schell 1985), which may explain the higher lipid readings, as less lipid energy stores would have been used prior to fluorescence quantification. 
The relationship between swimming behaviour and neutral lipid stores need further investigation, encompassing a larger range of cercariae species, coupled with close observation of their swimming patterns to determine if these observations have the potential to be robust.

Another possible reason for differences in lipid levels and localization among cercariae may be related to buoyancy and position in the water column. Neutral lipids in calanoid copepods are often considered to have a role in the regulation of buoyancy (reviewed by Sargent and Henderson, 1986). Neutral lipids provide up thrust in the water as they have a specific gravity of less than water and therefore are very effective in providing buoyancy (Sargent and Henderson 1986). Haematoloechus sp. cercariae had slightly higher lipid levels in the tail than body, and they, along with armatae-type cercariae typically reside lower in the water column or along the substrate. Armatae-type cercariae contained very low lipid levels, which may help them sink, however, Haematoloechus $s p$. contained high lipid levels. Echinostoma $s p$. exhibit differences in habitat preference related to species, as Echinostoma caproni prefer shallow water (Haas et al. 2008), and Echinostoma revolutum prefer deeper water (Loy et al. 2001). These species-specific preferences may have partially accounted for the lipid levels, as the Echinostoma sp. investigated here may have preferred deep water to locate target hosts, and therefore, reduced lipids would have counteracted buoyancy in the water column. Strigeid-type cercariae typically float more often than actively swim once emerged, and their tail hangs in an acute and downward angle to the body (Cort 1937). The strigeid-type body contained higher lipid levels than the tail, which may explain the lower tail position, as it doesn't contribute to their upright floating position in the water column. Further investigations into the potential for lipids to provide buoyancy within cercariae and aid in host-seeking behaviour are needed to determine if lipids have a major role in the position of cercariae within the water column.

The sensitivity of the Nile red method allows for the optical-binning technique to be effective in investigating individual differences. A similar conclusion was reached in a previous study estimating seasonal copepod lipids using an optical-digital measurement and an Iatroscan measurement, where individual variability was detectable in the optical-digital method (Arts and Evans 1991). Therefore, an obvious strength of the optical method is its sensitivity to individual variation which allows for small, but nonetheless important, differences in lipid levels to be detected among individuals (Arts and Evans 1991). For instance, the optical-binning technique allows for the investigation of individual lipid differences related to factors such as location, 
temperature, species/type, etc., where collecting thousands of cercariae for each factor or treatment would be onerous. The development of such techniques is thus important as they provide an independent, rapid, and economical method to assess lipid droplets at the individual level (Arts and Evans 1991). In addition, this approach provides information on the deposition and localization of lipids within the body segments of cercariae to better understand their likely function. This method is not limited to cercariae, but is likely also well suited to investigate lipid energy storage patterns and amounts in other life stages of parasites such as trematode rediae or sporocysts.

Variability in lipid levels among the different cercariae species/types may have also been driven by differences in the lipid composition of the intermediate snail host, which influences the lipid stores in the emergent parasites as cercariae rely solely on their snail host tissue for their energy reserves and lipids (Lunetta and Vernberg 1971; Southgate 1970; Fried et al. 1993). Lipid rich diets were found to alter the free sterol composition of the rediae tissue (containing developing cercariae), however no observed changes in TAG were detected (Fried et al. 2001), suggesting that a variety of factors affect parasite lipid levels. Haematoloechus sp. (which had that highest lipid levels) and strigeid-type were shed from infected L. stagnalis snail hosts, while armatae-type, $R$. ondatrae and Echinostoma sp. cercariae were shed from H. trivolvis snail hosts. Freshwater snails have been less extensively studied than their marine gastropod counterparts with respect to lipids; however, they are thought to similarly exhibit a diverse array of FA and lipids based on species-type (Joseph 1982). Studies of freshwater gastropods have found differences in lipids driven by thermal adaptation as well as environmental stress and may have accounted in the lipid variability in cercariae lipids detected in my study, as snails were collected from two very different climates; California and southern Ontario (Misra et al. 2002; Deschaseaux et al. 2010). Haematoloechus sp., R. ondatrae and Echinostoma sp. cercariae consume the ovotestis region of their hosts to obtain nutrients for their growth and development (Sorensen and Minchella 1998). Therefore, differences in lipid composition may arise from intrinsic variation in snail diet, metabolism and physiology (Leicht 2014). Additionally, $H$. trivolvis snails were collected from ponds in California, and L. stagnalis snails were originally from ponds in the southern Ontario region, where the composition of their diet and environmental factors would likely have led to differences in tissue composition (Lombardo 2002). Once the snails were brought into the lab, they were fed identical diets for upwards of 3 
weeks before cercarial shedding. However, intrinsic differences in environment may have affected snail composition, as estivation and starvation significantly decrease the lipid composition, and specifically TAG reserves in snails, and therefore environmental stress prior to collection may have attributed to tissue differences (White et al. 2006). As the region in California where the snails were collected (Pleasanton Ridge) experiences dryer and hotter summers than Southern Ontario, the H. trivolvis snails collected from the California ponds may have experienced higher levels of environmental stress and therefore a decreased lipid composition, as increased temperatures and warming cause significant decreases in gastropod lipids (Valles-Regino et al. 2015).

In addition to the individual-level approach in lipid estimation discussed above, an aggregate GC sample investigating the FA composition of $R$. ondatrae cercariae was also conducted. This technique lacks the ability to determine individual differences in cercariae as a single GC sample for this species consisted of $\sim 30,000$ cercariae to achieve a target dry weight of $\sim 15 \mathrm{mg}$ dry weight (DW). However, the sheer number of parasites aids in equalizing differences among individuals and represents a robust average, per cercariae, lipid level. As such, this is the first definitive FA profile for cercariae of any digenean trematode, and the first quantitative data on the FA present within any cercariae. Gas chromatographic efforts have been attempted previously on an aggregate of 60,000 S. mansoni cercariae, where total lipids were found to be $0.0133 \mu \mathrm{g}$ per S. mansoni cercariae (Smith et al. 1966). Those results were comparable to the $R$. ondatrae cercariae in this study that had $0.0317 \mu \mathrm{g}$ per cercariae. Previous efforts have utilized high-performance thin-layer chromatography (HPLC) to quantify the free sterols in trematodes but have focused less on the quantification of other lipids (Marsit et al. 2000a). HPLC was used to quantify free fatty acids (FFA) in S. mansoni (Schariter et al. 2002), and total FA levels in cercariae have been previously reported for three other digeneans: Z. lunata, at a level of $160 \mathrm{ng}$ per cercariae; S. mansoni at a level of $4.6 \mathrm{ng}$ per cercariae; and Echinostoma caproni at a level of $0.53 \mathrm{ng}$ (Marsit et al. 2000a,b, Schariter et al. 2002). In the present study, I report a value of $14.99 \mathrm{ng}(0.01499 \mu \mathrm{g})$ per cercaria for $R$. ondatrae, which is comparable to the previously published studies mentioned above. The cercariae of $Z$. lunata are large compared to that of $S$. mansoni, accounting for the large disparity in total FA values (Schariter et al. 2002). Riberioia ondatrae cercariae are larger than E. caproni and S. mansoni, therefore their size could in part 
account for the relatively higher amounts of total lipids and FA in Z. lunata and $R$. ondatrae cercariae.

Significant quantities of SFA (including palmitic [16:0] and stearic [18:0] acids), MUFA (including oleic acid [18:1n-9]) and n-3 PUFA (including 20:3n-3, ALA [18:3n-3], EPA, and DHA), and n-6 PUFA (including LNA [18:2n-6] and ARA) were found in individual cercaria of $R$. ondatrae (Table 2.1). GC analysis of $S$. mansoni cercariae identified 25 components, with the major fatty acids being palmitic, stearic, oleic and linoleic acid. The 20 or more carbon compounds overlapped in their analysis, therefore their identification and labelling were speculative. However, they identified 20:4 (3.6\%) and 20:5 (1.3\%) FA, which compared to $R$. ondatrae cercariae were as follows 20:4n-6 (1.66) and 20:5n-3 (5.27) (Table 2.1). The function of FA in the free-living intramolluscan stage is speculative; however, they have been suggested to contribute to buoyancy, energy reserves, metabolic waste products as well as serve as a chemo-attractant for larval trematodes (Marsit et al. 2000a; Fried et al. 2009). Although lipids have an important role in the survival of cercariae, both cestodes (tapeworms) and trematodes (flatworms) are thought to depend on an exogenous supply of these compounds for the biosynthesis of their complex lipids (Meyer et al. 1970; Ginger and Fairbairn 1966; Jacobsen and Fairbairn 1967). While cercariae depend on exogenous sources of lipids, Mandlowitz et al. (1960) revealed that $S$. mansoni cercariae contain lipases, suggesting that they can convert tripalmitin to palmitic acid and utilize host lipids. To fulfill their lipid requirements, cercariae are thought to uptake FFA bound to serum proteins within hosts, or FA derived from cellular triglycerides and phospholipids in host cell membranes via their tegument or ingestion (Chiang and Caulfield 1989; Furlong et al. 1995). Fatty acids are also thought to pass through the cercarial tegument and act as pre-cursors in the synthesis of a wide variety of eicosanoids (Fusco et al. 1985). When exposed to linoleate, S. mansoni cercariae produced eicosanoids coeluting with prostaglandin $\mathrm{E}_{2}, \mathrm{D}_{2}$ and $\mathrm{A}_{2}$, leukotriene $\mathrm{B}_{4}$, and 5-hydroxyeicosatetraenoic acid standards as well as immunoreactive prostaglandin $\mathrm{E}_{1}$, and $\mathrm{E}_{2}$, and 5- and 15- hydroxyeicosatetraenoic acids (Fusco et al. 1985). Eicosanoid synthesis along with an active arachidonate metabolism are essential in the invasion, transformation, and the immune evasion processes critical to establishment within their next host (Salafsky et al. 1984; Fusco et al. 1985; Fusco et al. 1986; Salafsky and Fusco 1987). Eicosanoids are signaling molecules made from the oxidation of ARA or other 20-carbon long PUFA and play an important role in immune function (Stanley and 
Miller 2006). ARA, as well as other LC-PUFA such as EPA, are highly bioactive compounds that function in immunity and act in mediating and coordinating cellular and humoral responses at specific and crucial moments (e.g., immediate reactions to infection) (Fusco et al. 1985; Stanley and Kim 2014).

A recent study identified $121 \omega x$ desaturase sequences within Cnidara, Rotifers, Mollusca, Annelida, and Arthropoda, indicating that they possess the genetic machinery to synthesize biologically active n-3 FA (Kabeya et al. 2018). Cercariae belong to the Phylum Platyhelminthes making them closely related to the clade Gnathifera, which includes the Phylum Rotifera, and which was identified to contain the necessary sequences (Edgecombe et al. 2011; Kabeya et al. 2018). The observation that cercariae contain FA metabolites and lipases as well as the potential for cercariae to contain the genetic machinery to synthesize de-novo FA, indicates promising potential for an active FA metabolism (Meyer et al. 1970, Furlong and Caufield 1989; Furlong 1991; Edgecombe et al. 2011; Kabeya et al. 2018). The potential ability to modify FA chain length implies that cercariae may serve as trophic upgraders, whereby they can convert shorter chain FA into longer LC-PUFA and which serve bioactive functions (Hiltunen et al. 2017). Specifically, LC-PUFA are trophically-transferred and selectively retained at higher trophic levels as they provide physiologically vital functions to all vertebrates (Arts et al. 2001). Identifying significant quantities of EFA, such as EPA and DHA, in cercariae indicates their ability to contribute these essential FA to aquatic ecosystems. A direct supply of EPA and ARA provides higher trophic level organisms with important precursors for eicosanoid synthesis (Schmitz and Ecker 2008; Scholtz et al. 2016), which have critical functions. For example, these EFA have vital roles in Daphnia and copepod reproduction (Demott and Müller-Navarra 1997; Chen et al. 2012), and serve as key mediators and regulators of inflammation and immunity in vertebrates (Calder 2010). Additionally, EPA is vital to the somatic growth and reproduction of herbivorous zooplankton, and a key driver of aquatic ecosystem productivity (Müller-Navarra 1995; Müller-Navarra et al. 2000).

My finding that $R$. ondatrae cercariae contain significant quantities of EPA and DHA, coupled with the immense biomass of cercariae, highlights their potential to contribute significant amounts of nutritionally-important resources to consumers in aquatic ecosystems. Kuris et al. (2008) quantified free-living cercariae in three California estuaries and found that the biomass of their annual production was three to tenfold that of the winter bird biomass, a top 
ecosystem predator. Additionally, Soldánová et al. (2016) estimated that 4.65 tons of cercariae are emitted annually into a typical freshwater pond; equivalent in mass to a large Asian elephant! The annual biomass (DW) of $R$. ondatrae cercariae was estimated in a California pond by Preston et al. (2013) using the calculated standing biomass of cercariae, as well as speciesspecific densities of infected snails multiplied by species-specific average daily outputs and biomass of each cercariae. Consequently, the annual cercarial biomass of $R$. ondatrae in that California pond was calculated to be $156 \mathrm{mg} / \mathrm{m}^{2} / \mathrm{yr}^{-1}$ (DW) (Preston et al. 2013). As the $R$. ondatrae cercariae used in this study were obtained from ponds in the same region, these estimates reflect the potential biomass of the collected cercariae from their respective ponds. From Preston et al. (2013) biomass estimates, I calculated the amount of EPA and DHA (mg DW) that $R$. ondatrae cercariae in the same California pond may represent using my FA data from $R$. ondatrae (Table 2.3), as well as cercariae mass (DW) and estimated numbers of cercariae (Dumont et al. 1975; Lambden and Johnson 2013) (Table 2.3). Using these parameters, I determined that cercariae may contribute $\sim 24 \mathrm{mg} / \mathrm{m}^{2} / \mathrm{yr}^{-1}$ of EPA and $\sim 0.4 \mathrm{mg} / \mathrm{m}^{2} / \mathrm{yr}^{-1}$ of DHA annually in the California pond studied by Preston et al (2013) (Table 2.3). Furthermore, $R$. ondatrae cercariae were found to contain $8.08 \%$ lipid (DW) and when compared to Daphnia, whose lipid content can drastically range based on diet from $11.1 \%$ to $22.1 \%$ (DW) (Macedo \& Pinto-Coelho 2000), may therefore present a lipid rich food source. When compared to Daphnia, cercaria are not encased in a tough chitin exoskeleton and are therefore potentially an easier food source for predators to process, digest and assimilate. Further investigation into the potential for cercariae to offset temporal zooplankton variation within these pond systems, and a direct comparison between $R$. ondatrae and Daphnia FA composition, would further highlight the direct comparability of these two food types.

Given that EPA and DHA are limiting resources in aquatic ecosystems, the discovery that cercariae contain, and are supplying considerable quantities of, EPA and DHA to aquatic predators is important for the growth and productivity of aquatic ecosystems. Further, it highlights the ability for cercariae to serve as a bridge for the transfer of EPA and DHA across the plant-animal interface, which is vital in ensuring that these nutrients are more accessible for consumption by aquatic predators. The identification of the novel source, coupled with their considerable biomass and ability to serve as prey highlights the importance of their consideration in food web models and nutrient transfer in aquatic ecosystems. 
Table 2.3: Estimating the annual contribution in dry weight (DW) of EPA and DHA from Ribeiroia ondatrae cercariae in a California pond. Trematode cercariae biomass estimates in California pond obtained from Preston et al. (2013), mass of individual cercariae (DW) determined by Lambden and Johnson (2013) fatty acid composition of cercariae determined in Chapter 2. of this thesis, by K. Mckee (unpublished data).

\begin{tabular}{|c|c|c|c|c|}
\hline $\begin{array}{l}\quad R \text {. ondatrae } \\
\text { cercariae annual } \\
\text { DW biomass in } \\
\text { California pond } \\
\text { (Preston et al. } \\
\text { 2013) }\end{array}$ & $\begin{array}{l}\text { Mass (DW) } \\
\text { of } R \text {. ondatrae } \\
\text { cercariae } \\
\text { (Lambden and } \\
\text { Johnson 2013) }\end{array}$ & $\begin{array}{c}\text { Number of } \\
\text { cercariae in pond } \\
\text { (biomass/mass } \\
\text { per cercariae) }\end{array}$ & $\begin{array}{c}\mu \mathrm{g} \text { DW fatty acids } \\
\text { per } R \text {. ondatrae } \\
\text { cercariae }\end{array}$ & $\begin{array}{c}\text { Total fatty acids in } \\
\text { California pond ( } \mu \mathrm{g} \text { fatty } \\
\text { acids per cercariae*number } \\
\text { of cercariae) }\end{array}$ \\
\hline \multirow{2}{*}{$156 \mathrm{mg} / \mathrm{m}^{2} / \mathrm{yr}^{-1}$} & \multirow{2}{*}{$0.0052 \mathrm{mg}$} & \multirow{2}{*}{$\begin{array}{c}30,000 \text { cercariae/ } \\
\mathrm{m}^{2} / \mathrm{yr}^{-1}\end{array}$} & $\begin{array}{c}\mathrm{EPA}=0.000791 \\
\mu \mathrm{g} / \text { cercariae }\end{array}$ & $\mathrm{EPA} \approx 24 \mathrm{mg} / \mathrm{m}^{2} / \mathrm{yr}^{-1}$ \\
\hline & & & $\begin{array}{c}\mathrm{DHA}=0.000013 \\
\mu \mathrm{g} / \text { cercariae }\end{array}$ & $\mathrm{DHA} \approx 0.4 \mathrm{mg} / \mathrm{m}^{2} / \mathrm{yr}^{-1}$ \\
\hline
\end{tabular}




\title{
CHAPTER 3: INFLUENCE OF RIBEIROIA ONDATRAE INFECTION ON FATTY ACID PROFILES OF HELISOMA TRIVOLVIS HOST SNAILS
}

\author{
Keira M. McKee, Janet Koprivnikar, Micheal T. Arts
}

This chapter is in preparation to be submitted to the Journal of Parasitology.

\begin{abstract}
This study examined the effects of larval trematode infection on the fatty acid content of host Helisoma trivolvis snails infected with rediae of Ribeiroia ondatrae. Uninfected snails were used as controls. All snails (infected and uninfected) were dissected into two sections, the digestive gland-gonad (DGG) complex (refered to body tissue throughout) and the head/foot region, in order to investigate the effect of infection. Infection with $R$. ondatrae significantly increased the total lipid content in infected snails by $41.1 \%$. Infected snails contained significantly higher proportions (\% of total identified FA) of 14:0, 22:2n-6, nervonic acid (24:1n-9), DHA (22:6n-3) and SFA. Infected snails also had higher mass fractions ( $\mu \mathrm{g}$ of FA per mg of sample DW) than uninfected snails for the following FA; 18:2n-6, 18:3n-3, 22:3n-3 and EPA (20:5n-3). The DGG of infected snails also contained significantly higher absolute fractions of DHA. Differences in the lipid and fatty acid composition due to infection probably reflect differences in the metabolic activity of the host or parasite, secretion of lipid from the parasite themselves or fatty acid mobilization to the infection site.
\end{abstract}

\section{Introduction}

Snails experience seasonally fluctuating photoperiods and changes in food quality and quantity, and which may be combined with, and/or interspersed with, periods of fasting, starvation, and/or estivation (Lustrino et al. 2010; Pinheiro et al. 2009; Tunholi-Alves et al. 2011). All of these processes may impact lipid metabolism and hence lipid (including fatty acid FA) profiles in snails. Other factors may also affect FA profiles in snails. For instance, freshwater snails typically serve as the first intermediate hosts for trematode parasites (see Chapter 1, Fig. 1.2) and different infection type may induce patho-biochemical changes on their snail hosts. Inside snails, larval trematodes develop from invading miracidia into sporocysts or rediae that undergo asexual reproduction, with sporocysts absorbing (e.g., from the hemolymph) host-derived nutrients and rediae consuming host tissue directly using a mouthpart specifically 
designed for this purpose (Sorensen and Minchella 1998). Within sporocysts and rediae, the next stage of the trematode life cycle is produced - free-living cercariae that emerge from snails when mature. Consequently, cercariae derive all of their nutrients, from their snail hosts, during the rediae or sporocyst stage (Johnson et al. 2004). The presence of these infections within gastropods induces patho-biochemical changes in the composition of host tissue and hemolymph; however, the effects of trematode infection on snail lipid composition have been contradictory (Fried et al. 1989, 1993a,b, 1998; Sherma and Fried 1990). Among these changes are parasite-induced alterations to the lipid composition of the snail host (Fried et al. 1989, 2001).

Various chromatographic techniques (thin-layer- and gas-liquid-chromatography; TLC and $\mathrm{GC}$, respectively) have been used to investigate the effects of larval trematode infection on the neutral lipid composition of first intermediate hosts (snails) encompassing several host-parasite relationships (see Fried and Sherma 1990). However, as noted above, the results have varied based on snail and infection type, with reports of both increased and decreased levels of the triacylglycerol fractions (TAG) in the snails. Fried et al. (1989, 1993a), Shetty et al. (1992) and Bandstra (2006) reported significant reductions in the TAG fraction of the digestive gland-gonad (DGG) complex (the snail tissue structure in which rediae and sporocysts develop), while others have reported significant increases in the TAG fraction (Thompson 1987; Fried et al. 1998c; Fried and Sherma 1990). These differences indicate that trematode infections markedly influence the lipid composition of snail bodies but are likely dependent on the species involved (Fried and Sherma 1990). For example, Fried et al. (1989) investigated the effects of larval Echinostoma caproni (Richard, 1964) infection on the neutral lipid content of the DGG of Biomphalaria glabrata (Say, 1818) snails using semi-quantitative high performance thin-layer chromatography (HPLC), and reported higher TAG fractions in controls than infected DGG, concluding that larval trematodes reduced the fat depot in the DGG of infected snails. Infections with other trematode and snail species, such as Echinostoma trivolvis (Cort, 1914) in Helisoma trivolvis (Say, 1817) snails, paradoxically, showed elevated TAG fractions in the DGG of infected snails (Fried and Sherma 1990).

General gastropod anatomy consists of a muscular head/foot region and a large visceral mass which contains the DGG. Hereafter, the muscular head/foot region is referred to as head/foot tissue, and the visceral mass including the DGG is referred to as DGG. The entire snail tissue, 
inclusive of both the head/foot and DGG tissue is referred to here as body tissue. Fried et al. (1993a) reported differences in certain FA constituents in the head/foot tissue as well as the DGG complex of $B$. glabrata snails infected with E. caproni rediae. They reported a marked decrease in the levels of saturated fatty acids (SFA) in the head/foot tissue of infected snails, and increased levels in the DGG complex. Additionally, the concentration of docosahexaenoic acid (22:6n-3; DHA) decreased in the body tissue of infected snails. Fried et al. (1993b) further investigated the effect of snail species on trematode-related patho-biochemistry using $H$. trivolvis snails that were also infected with E. caproni. The DGG of infected H. trivolvis snails had significantly higher levels of stearic acid (18:0), hexatrienoic acid (16:3n-4), and DHA compared to uninfected controls, but the DGG of uninfected snails showed significantly higher levels of 20:2 NMID (non-methylene-interrupted dienoic aka polymethylene-interrupted dienoic = PMID) and adrenic acid (22:2n-6) than uninfected snails. Differences in FA profiles between infected and uninfected H. trivolvis and B. glabrata snail hosts, infected with the same parasite species, were suggested to be driven primarily by the available lipid pools or metabolic activity of the immediate host, which influences the composition of the DGG tissue, as it harbors developing parasites (Tunholi et al. 2011).

The effects of trematode infection vary based upon host species, however these changes may also depend on the trematode species involved. Fried et al. (1998) used thin-layer chromatography (TLC) to investigate the neutral lipid content of the DGG complex of $H$. trivolvis snails infected with four different larval trematode species/types. As cercariae develop in two different types of developmental structures, rediae were investigated for Ribeiroia sp. and Zygocotyle lunata infections, and sporocysts for Spirorchis sp. and snails infected with an armatae-type xiphidiocercaria. Each trematode infection type resulted in a distinct neutral lipid profile (only the main lipid classes were investigated; compounds within specific lipid classes were not investigated) in the snails, and the profiles differed between infected and uninfected snails for each type. Visual observations of the chromatographs showed that all four-trematode species caused significant elevations in the triacylglycerol fraction in the DGG of their host snails compared with the uninfected controls. Oil Red O (ORO) histochemical techniques also identified increased levels of neutral lipids in the DGG of the infected snails.

Bandstra et al. (2006) examined the effect of E. caproni infection on the neutral lipid content of B. glabrata snails. They identified that the major lipid groups found in the whole bodies and 
DGG complexes of both the infected and uninfected snails were free sterols (FS), and triacylglycerides (TAG). The amount of TAG in infected DGG were also identified to be significantly less than that of the uninfected DGG tissue.

There is significant variation in previously-published findings regarding the effects of larval trematode infection on the patho-biochemistry of intermediate snail hosts. Neutral lipid content and fatty acid (FA) composition varies based on snail species, larval trematode species/type, and snail diet; however, further investigations into different host-parasite relationships are necessary to identify overarching patterns. Lipids are the primary energy currency in aquatic ecosystems and are essential to the health and survival of all vertebrates (Müller-Navarra 1995; Arts et al. 1999, 2009; see Chapter 1). Trematode parasites alter the FA composition of their intermediate hosts, which may alter their metabolism and ability to survive other stressors (i.e. temperature, eutrophication), as well as alter their behavior including, predator avoidance tactics or foraging patterns (Levri and Lively 1996; Levri 1999; Bernot and Turner 2001; Bernot 2003). Thus, the purpose of my study was to investigate, using GC analysis, the effect of Ribeiroia ondatrae infection on the FA composition of the total lipid extracts of infected and uninfected H. trivolvis snails. I hypothesized that trematode infection would increase the total lipid fractions, as this was previously observed in the host-parasite relationship for H. trivolvis and $R$. ondatrae. However, individual FA molecules were not determined in the previous study therefore I am investigating the effect of infection on specific fatty acid molecules as well.

\section{Materials and Methods}

\section{Field Collection and Animal Care}

Aquatic snails (H. trivolvis) were collected from two ponds in Pleasanton Ridge Regional Park in California $\left(37.6154094^{\circ} \mathrm{N},-121.8823708^{\circ} \mathrm{W}\right)$. Aquatic snails were collected and screened for infection based on a previously described protocol (see Chapter 2, Field Collection and Animal Care). Infected snails were shed to confirm the presence of $R$. ondatrae infection, and the cercariae species was confirmed by microscopic examination of shed parasites. Snails were then frozen prior to dissection. Nine $R$. ondatrae infected snails and nine uninfected snails were used for gas chromatography (GC) analysis. 


\section{Lipid Analysis}

Whole snails were dissected, and each individual snail was analyzed in two parts. Snails were placed in a petri dish and after the shell was gently crushed the body was removed with forceps. Eighteen snails (nine uninfected and nine infected) were randomly selected from a pool of 50 infected and 50 uninfected snails. The DGG complex, which occupies the posterior one-third of the body was removed from the rest of the body, separating each snail into two parts; the DGG and the head/foot region. The DGG of infected snails is largely trematode tissue but may have trace amounts of host tissue as it was difficult to separate all host tissue. The DGG was weighed, frozen at $-80^{\circ} \mathrm{C}$ and placed in a microcentrifuge tube for freeze drying. The remaining head/foot regions of both infected and uninfected snails were also weighed, placed in tubes, and frozen prior to freeze drying. Prior to lipid extraction, the tissue was ground with a mortar and pestle with the addition of a few drops of liquid nitrogen to render the tissue to a fine powder. The lipid extraction and GC procedure were previously described in Chapter 2 (see Chapter 2, Lipid Extraction).

Digenetic trematodes castrate their gastropod hosts, therefore the rediae (harboring numerous developing cercariae), are strictly localized to the gonadal tissue in the posterior end of the snail, leaving the head/foot region free of infected tissue (Baudoin 1975). Separating each snail into two sections (DGG and head/foot region) allowed me to investigate the effect of trematode infection on FA composition in different snail (host) tissues. Previous studies have used similar methods to investigate the effects of trematode infection on the FA composition of the total lipid extract of gastropod hosts (Fried et al. 1993 a,b; Fried et al. 1998c, 2001 Bandstra et al. 2006)

\section{Statistical Analysis}

Prior to data analysis, all of the data were tested for normality, homogeneity of variance and independence of residuals. Based on these tests a log-transformation was applied to the percent content data (\% composition) for each FA type since this transformation resulted in normally distributed data. Additionally, the data was also presented on an absolute basis ( $\mu$ g of FA per mg of sample (DW)), and no transformations were applied to the data. Gravimetric data was also presented with no transformations. A factorial analysis of variance (ANOVA) model was used to compare the main effects of infection status and tissue type, and the interaction effect between infection status and tissue type on individual snail FA proportions as well as the total average lipids (mg of total lipid/mg of tissue (DW)). Infection status was defined by one of two 
categories ( $R$. ondatrae infection present or absent), as was tissue type (DGG or head/foot tissue). Effects were judged to be statistically significant at the $p<0.05$ level and only significant results were discussed.

\section{Results}

Infection with $R$. ondatrae parasites had a significant effect on the gravimetric results for average lipid (mg of lipid / mg of tissue) content of $H$. trivolvis snail tissue (Fig. 3.1, Table 3.3). Infected snails had $41.1 \%$ more lipids overall than uninfected snails $(\mathrm{P}=0.0001)$ (Table 3.1). There was no difference between the tissue types with respect to total lipid nor was there any interaction between the two factors. Infected $H$. trivolvis snails contained significantly higher proportions of the following FA: 14:0 (3.31 vs. $1.42 \%), 22: 2 n-6$ (0.43 vs. $0.25 \%), 24: 1 n-9$ (nervonic acid) (0.33 vs. $0.13 \%$ ), DHA (0.78 vs. $0.057 \%$ ), and sum SFA (64.8 vs. $56.2 \%)$ (Table 3.3).

Tissue type affected the proportion of 20:4n-6 (arachidonic acid; ARA) (18.8 vs. $11.2 \%$ ), with head/foot tissue containing a higher proportion compared to DGG tissue irrespective of infection status (Table 3.1, Table 3.3). Tissue type also affected the composition of 19:0 (1.4 vs. $0.48 \%$ ) 20:1n-12 (5.3 vs. $2.0 \%$ ) and 20:3n-3 (11.3 vs. $6.4 \%$ ) given that DGG tissue had higher proportions of each FA than the head/foot tissue (Table 3.1, Table 3.3).

Infection status interacted with tissue type to affect certain FA proportions. This was seen for the proportion of 18:3n-6 (gamma linolenic acid; GLA) (Table 3.3). The follow-up Tukey LSD post-hoc test revealed that the infected snail DGG (0.62\%) and uninfected snail feet $(0.24 \%)$ contained on average lower GLA proportions, while uninfected DGG $(0.93 \%)$ and infected feet $(0.67 \%)$ contained on average higher proportions (Table 3.1, Table 3.3).

The DGG complex of $H$. trivolvis snail had higher absolute mass fractions ( $\mu$ g of FA per mg of sample (dry weight (DW)) than the head/foot tissue respectively for; 16:0 (8.8 vs. $4.2 \mu \mathrm{g}$ of FA per mg of sample DW), 17:1n-7 (0.54 vs. $0.33 \mu \mathrm{g}$ of FA per mg of sample DW), 22:0 (0.66 vs. $0.26 \mu \mathrm{g}$ of FA per mg of sample DW) and 20:3n-3 (5.3 vs. $1.9 \mu \mathrm{g}$ of FA per mg of sample DW) (Table 3.2, Table 3.4). Infection type was insignificant, and there was no interaction between the two factors. 
Infected H. trivolvis snails had higher mass fractions ( $\mu$ g of FA per mg of sample DW) than uninfected snails for the following FA respectively; 18:2n-6c (3.3 vs. $1.2 \mu \mathrm{g}$ of FA per mg of sample DW), 18:3n-3 (12.4 vs. $4.0 \mu \mathrm{g}$ of FA per mg of sample DW), 22:3n-3 (0.92 vs. $0.34 \mu \mathrm{g}$ of FA per mg of sample DW), 20:5n-3 (5.7 vs. $2.8 \mu \mathrm{g}$ of FA per mg of sample DW) (Table 3.2, Table 3.4). Tissue type was insignificant, and there was no interaction between the two factors.

Infection status interacted with tissue type for the FA mass fractions below (Table 3.2, Table 3.4). Infected $H$. trivolvis snail DGG $(0.17 \mu \mathrm{g}$ of FA per mg of sample DW) had less $18: 3 \mathrm{n}-6$ (GLA) than uninfected DGG tissue ( $0.27 \mu \mathrm{g}$ of FA per mg of sample DW), and infected head/foot $(0.30 \mu \mathrm{g}$ of FA per mg of sample DW) tissue had higher quantities than uninfected head/foot $(0.051 \mu \mathrm{g}$ of FA per mg of sample DW). Infected DGG tissue (1.5 $\mu \mathrm{g}$ of FA per mg of sample DW) had similar quantities of 20:3n-6, to infected head/foot (1.8 $\mu \mathrm{g}$ of FA per mg of sample DW), and uninfected DGG (1.6 $\mu \mathrm{g}$ of FA per mg of sample DW), however uninfected head/foot had less $(0.31 \mu \mathrm{g}$ of FA per mg of sample DW). Infected DGG $(0.043 \mu \mathrm{g}$ of FA per mg of sample DW) and head/foot (0.079 $\mu \mathrm{g}$ of FA per mg of sample DW) tissue had similar quantities of 22:4n-6, to uninfected DGG (0.084), however uninfected foot $(0.57 \mu \mathrm{g}$ of FA per $\mathrm{mg}$ of sample DW) had higher quantities. Infected DGG $(0.011 \mu \mathrm{g}$ of FA per mg of sample DW), infected head/foot (0.014 $\mu \mathrm{g}$ of FA per mg of sample DW) tissue and uninfected DGG (0.021) had similar quantities of 22:5n-3, uninfected foot had higher ( $0.18 \mu \mathrm{g}$ of FA per mg of sample DW). Infected DGG tissue had the highest mass fraction of 22:6n-3 (DHA) (0.15 $\mu \mathrm{g}$ of FA per mg of sample DW), infected head/foot ( $0.008 \mu \mathrm{g}$ of FA per mg of sample DW), uninfected DGG $(0.010 \mu \mathrm{g}$ of FA per mg of sample DW) and uninfected head/foot $(0.008 \mu \mathrm{g}$ of FA per mg of sample DW) were lower (Fig. 3.2). 
Table 3.1: Mean \pm S.D. proportional fatty acid composition $(\% \mathrm{mg})$ and mass fraction of total lipid (mg of lipid/mg of tissue DW) in Helisoma trivolvis snails infected and uninfected by Ribeiroia ondatrae (DGG = digestive gland-gonad complex). Bold indicates significance at a level of $\mathrm{P}<0.05$. *

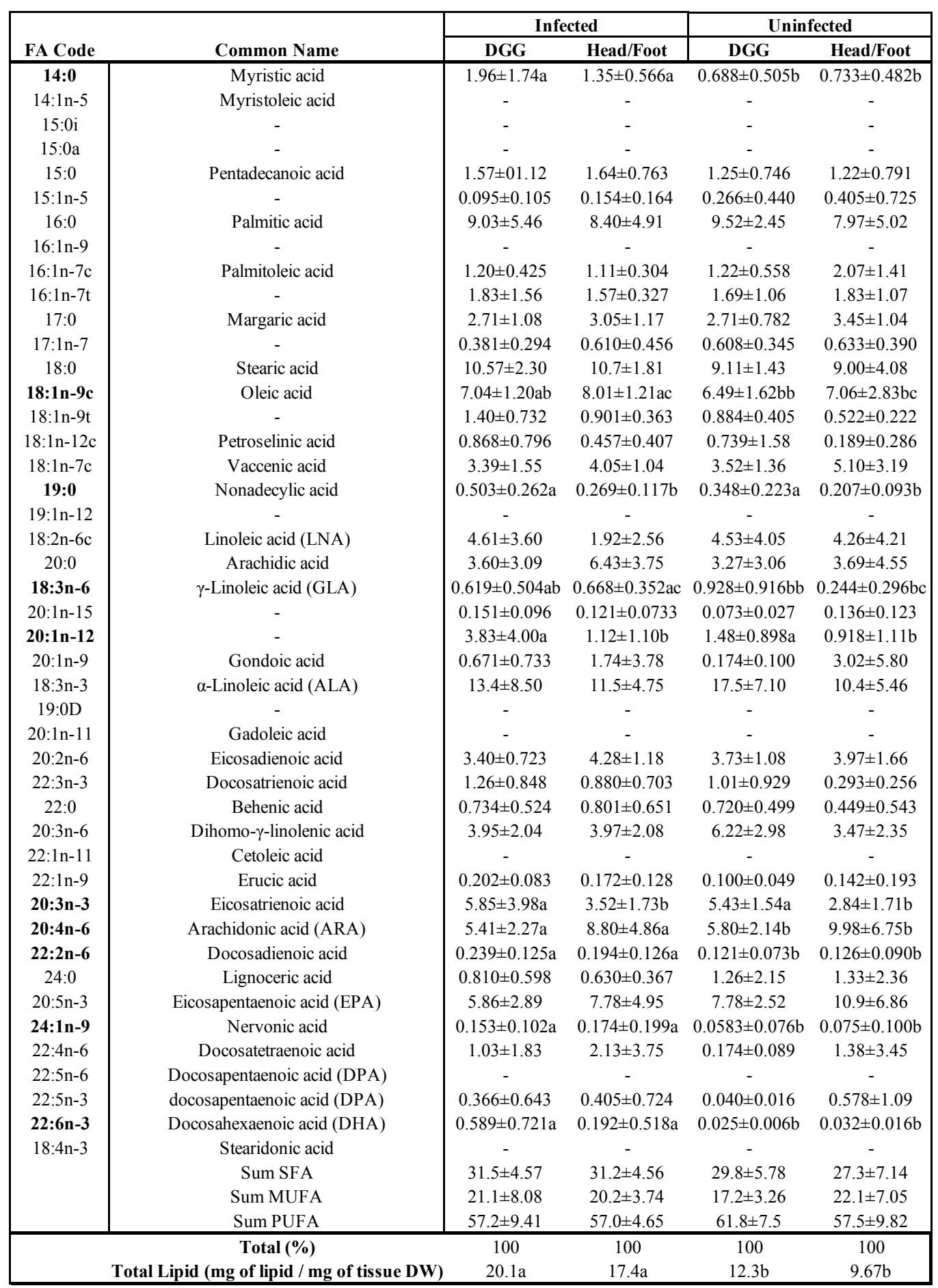

$*_{n}=9$ in all groups. 
Table 3.2: Mean \pm S.D. absolute fatty acid data ( $\mu$ g of FA per $m g$ of sample DW in Helisoma trivolvis snails infected and uninfected by Ribeiroia ondatrae (DGG = digestive gland-gonad complex). Bold indicates significance at a level of $\mathrm{P}<0.05$. *

\begin{tabular}{|c|c|c|c|c|}
\hline \multirow[b]{2}{*}{ FA Code } & \multicolumn{2}{|c|}{ Infected } & \multicolumn{2}{|c|}{ Uninfected } \\
\hline & DGG & Head/Foot & DGG & Head/Foot \\
\hline $14: 0$ & $0.558 \pm 0.552$ & $0.530 \pm 0.489$ & $0.456 \pm 0.674$ & $0.243 \pm 0.429$ \\
\hline $14: 1 n-5$ & - & - & - & - \\
\hline $15: 0 \mathrm{i}$ & - & - & - & - \\
\hline $15: 0 \mathrm{a}$ & - & - & - & - \\
\hline $15: 0$ & $0.506 \pm 0.690$ & $0.596 \pm 0.479$ & $0.804 \pm 1.12$ & $0.272 \pm 0.64$ \\
\hline $15: 1 n-5$ & $0.05 \pm 0.038$ & $0.050 \pm 0.038$ & $0.230 \pm 0.585$ & $0.053 \pm 0.072$ \\
\hline $16: 0$ & $4.27 \pm 3.70 \mathrm{a}$ & $3.71 \pm 2.69 \mathrm{~b}$ & $4.53 \pm 4.33 \mathrm{a}$ & $0.409 \pm 0.618 b$ \\
\hline $16: 1 n-9$ & - & - & - & - \\
\hline $16: 1 \mathrm{n}-7 \mathrm{c}$ & $0.454 \pm 0.334$ & $0.409 \pm 0.413$ & $0.645 \pm 0.628$ & $0.261 \pm 0.234$ \\
\hline $16: 1 n-7 t$ & $0.597 \pm 0.805$ & $0.580 \pm 0.460$ & $0.748 \pm 0.689$ & $0.417 \pm 0.619$ \\
\hline 17:0 & $0.791 \pm 0.615$ & $0.839 \pm 0.537$ & $1.20 \pm 1.03$ & $0.634 \pm 0.404$ \\
\hline $17: 1 n-7$ & $0.225 \pm 0.221 \mathrm{ab}$ & $0.288 \pm 0.226 \mathrm{ac}$ & $0.314 \pm 0.386 b b$ & $0.038 \pm 0.058 b c$ \\
\hline 18:0 & $3.65 \pm 3.54$ & $3.55 \pm 2.40$ & $2.29 \pm 1.50$ & $1.70 \pm 1.75$ \\
\hline $18: 1 n-9 c$ & $2.37 \pm 1.71$ & $2.57 \pm 1.81$ & $3.24 \pm 3.17$ & $1.22 \pm 0.953$ \\
\hline $18: 1 n-9 t$ & $0.454 \pm 0.597$ & $0.312 \pm 0.210$ & $0.324 \pm 0.271$ & $0.176 \pm 0.278$ \\
\hline $18: 1 n-12 c$ & $0.217 \pm 0.256$ & $0.103 \pm 0.135$ & $0.207 \pm 0.336$ & $0.075 \pm 0.092$ \\
\hline $18: 1 \mathrm{n}-7 \mathrm{c}$ & $1.15 \pm 1.14$ & $1.24 \pm 0.770$ & $1.84 \pm 1.93$ & $0.848 \pm 0.537$ \\
\hline 19:0 & $0.172 \pm 0.223$ & $0.110 \pm 0.115$ & $0.122 \pm 0.099$ & $0.072 \pm 0.122$ \\
\hline $19: 1 \mathrm{n}-12$ & - & - & - & - \\
\hline $18: 2 n-6 c$ & $2.26 \pm 2.60 \mathrm{a}$ & $1.02 \pm 1.58 \mathrm{a}$ & $0.693 \pm 0.662 b$ & $0.514 \pm 0.665 b$ \\
\hline $20: 0$ & $0.661 \pm 0.769$ & $1.69 \pm 1.92$ & $1.53 \pm 2.83$ & $0.877 \pm 0.774$ \\
\hline $18: 3 n-6$ & $0.172 \pm 0.205 \mathrm{ab}$ & $0.297 \pm 0.293 \mathrm{ac}$ & $0.273 \pm 0.344 \mathrm{bb}$ & $0.051 \pm 0.077 \mathrm{bc}$ \\
\hline $20: 1 n-15$ & $0.035 \pm 0.037$ & $0.029 \pm 0.020$ & $0.032 \pm 0.032$ & $0.035 \pm 0.040$ \\
\hline $20: 1 n-12$ & $0.645 \pm 0.602$ & $0.259 \pm 0.296$ & $0.687 \pm 0.822$ & $0.788 \pm 1.524$ \\
\hline $20: 1 n-9$ & $0.185 \pm 0.297$ & $0.249 \pm 0.622$ & $0.060 \pm 0.041$ & $0.519 \pm 0.597$ \\
\hline $18: 3 n-3$ & $6.73 \pm 10.4 \mathrm{a}$ & $5.65 \pm 4.23 \mathrm{a}$ & $2.99 \pm 2.91 \mathrm{~b}$ & $0.986 \pm 0.629 b$ \\
\hline 19:0D & - & - & - & - \\
\hline $20: 1 n-11$ & - & - & - & - \\
\hline $20: 2 n-6$ & $1.15 \pm 1.00$ & $1.28 \pm 0.884$ & $1.88 \pm 1.89$ & $0.687 \pm 0.409$ \\
\hline $22: 3 n-3$ & $0.569 \pm 0.569 \mathrm{a}$ & $0.346 \pm 0.320 \mathrm{a}$ & $0.297 \pm 0.216 b$ & $0.041 \pm 0.037 \mathrm{~b}$ \\
\hline 22:0 & $0.310 \pm 0.306 \mathrm{a}$ & $0.198 \pm 0.141 b$ & $0.348 \pm 0.355 \mathrm{a}$ & $0.059 \pm 0.050 \mathrm{~b}$ \\
\hline $20: 3 n-6$ & $1.48 \pm 1.41 \mathrm{ab}$ & $1.75 \pm 1.31 \mathrm{ac}$ & $1.55 \pm 0.843 \mathrm{bb}$ & $0.307 \pm 0.254 b c$ \\
\hline $22: 1 n-11$ & - & - & - & - \\
\hline $22: 1 n-9$ & $0.064 \pm 0.056$ & $0.042 \pm 0.035$ & $0.046 \pm 0.048$ & $0.037 \pm 0.040$ \\
\hline $20: 3 n-3$ & $3.03 \pm 4.14 \mathrm{a}$ & $1.60 \pm 1.10 \mathrm{~b}$ & $2.25 \pm 1.91 \mathrm{a}$ & $0.267 \pm 0.237 b$ \\
\hline $20: 4 n-6$ & $1.69 \pm 1.37$ & $2.26 \pm 1.66$ & $2.81 \pm 2.77$ & $1.66 \pm 0.665$ \\
\hline $22: 2 n-6$ & $0.065 \pm 0.071$ & $0.045 \pm 0.041$ & $0.041 \pm 0.041$ & $0.048 \pm 0.060$ \\
\hline $24: 0$ & $0.253 \pm 0.255$ & $0.225 \pm 0.203$ & $0.421 \pm 0.421$ & $0.094 \pm 0.093$ \\
\hline $20: 5 n-3$ & $2.87 \pm 2.14 \mathrm{a}$ & $2.85 \pm 1.73 b$ & $2.15 \pm 3.14 \mathrm{a}$ & $0.607 \pm 0.445 b$ \\
\hline $24: 1 n-9$ & $0.059 \pm 0.052$ & $0.026 \pm 0.031$ & $0.016 \pm 0.011$ & $0.024 \pm 0.019$ \\
\hline $22: 4 n-6$ & $0.043 \pm 0.030 \mathrm{ab}$ & $0.079 \pm 0.056 \mathrm{ac}$ & $0.084 \pm 0.085 b b$ & $0.572 \pm 0.559 \mathrm{bc}$ \\
\hline $22: 5 n-6$ & - & - & - & - \\
\hline $22: 5 n-3$ & $0.011 \pm 0.007 \mathrm{ab}$ & $0.014 \pm 0.010 \mathrm{ac}$ & $0.021 \pm 0.022 b b$ & $0.180 \pm 0.174 b c$ \\
\hline $22: 6 n-3$ & $0.148 \pm 0.129 a$ & $0.008 \pm 0.009 b$ & $0.010 \pm 0.008 \mathrm{~b}$ & $0.008 \pm 0.007 \mathrm{~b}$ \\
\hline $18: 4 n-3$ & - & - & - & - \\
\hline Sum SFA & $11.7 \pm 9.31$ & $11.3 \pm 8.0$ & $11.7 \pm 10.9$ & $4.36 \pm 3.25$ \\
\hline Sum MUFA & $6.43 \pm 5.4$ & $6.13 \pm 4.32$ & $8.37 \pm 8.08$ & $4.47 \pm 4.04$ \\
\hline Sum PUFA & $20.2 \pm 22.5$ & $17.2 \pm 11.6$ & $15.0 \pm 10.15$ & $5.93 \pm 2.27$ \\
\hline Total ( $\mu$ g of FA / mg of tissue DW) & 38.0 & 34.8 & 35.2 & 15.1 \\
\hline
\end{tabular}

$*_{n}=9$ in all groups. 
Table 3.3. Univariate factorial ANOVA results of the effects of infection and tissue type on the proportional fatty acid composition (\% mg) and mass fraction of total lipid (mg of lipid/mg of tissue DW) composition of Helisoma trivolvis snails.

\begin{tabular}{|c|c|c|c|c|c|c|}
\hline FA Code & Source & $\begin{array}{c}\text { Type III Sum of } \\
\text { Squares }\end{array}$ & df & Mean Square & F Value & $p$-value \\
\hline \multirow{3}{*}{$14: 0$} & Infection & 0.237 & 1 & 0.237 & 9.18 & 0.005 \\
\hline & Tissue & 0.002 & 1 & 0.002 & 0.088 & 0.769 \\
\hline & Interaction & 0.08 & 1 & 0.08 & 0.327 & 0.52 \\
\hline \multirow{3}{*}{$18: \ln -9$} & Infection & 0.078 & 1 & 0.078 & 7.393 & 0.010 \\
\hline & Tissue & 0.069 & 1 & 0.069 & 6.536 & 0.016 \\
\hline & Interaction & $1.97 \mathrm{e}-5$ & 1 & $1.97 \mathrm{e}-5$ & 0.002 & 0.966 \\
\hline \multirow{3}{*}{$19: 0$} & Infection & 0.011 & 1 & 0.011 & 3.462 & 0.072 \\
\hline & Tissue & 0.029 & 1 & 0.029 & 9.61 & 0.004 \\
\hline & Interaction & 0.097 & 1 & 0.097 & 0.482 & 0.493 \\
\hline \multirow{3}{*}{$20: 1 n-12$} & Infection & 0.151 & 1 & 0.151 & 2.12 & 0.155 \\
\hline & Tissue & 0.447 & 1 & 0.447 & 26.28 & 0.017 \\
\hline & Interaction & 0.058 & 1 & 0.058 & 0.817 & 0.373 \\
\hline \multirow{3}{*}{$20: 3 n-3$} & Infection & 0.001 & 1 & 0.001 & 0.21 & 0.886 \\
\hline & Tissue & 0.337 & 1 & 0.337 & 6.399 & 0.017 \\
\hline & Interaction & 0.034 & 1 & 0.034 & 0.644 & 0.428 \\
\hline \multirow{3}{*}{$22: 2 n-6$} & Infection & 0.010 & 1 & 0.010 & 7.494 & 0.010 \\
\hline & Tissue & 0.00 & 1 & 0.00 & .351 & 0558 \\
\hline & Interaction & 0.001 & 1 & 0.001 & .523 & 0.475 \\
\hline \multirow{3}{*}{$18: 3 n-6(G L A)$} & Infection & 0.012 & 1 & 0.012 & 0.725 & 0.401 \\
\hline & Tissue & 0.043 & 1 & 0.043 & 2.51 & 0.123 \\
\hline & Interaction & 0.074 & 1 & 0.074 & 4.32 & 0.046 \\
\hline \multirow{3}{*}{ ARA } & Infection & 0.003 & 1 & 0.003 & 0.082 & 0.776 \\
\hline & Tissue & 0.237 & 1 & 0.237 & 6.383 & 0.17 \\
\hline & Interaction & $2.67 \mathrm{e}-5$ & 1 & $2.67 e-5$ & 0.001 & 0.979 \\
\hline \multirow{3}{*}{ 24:1n-9 (Nervonic Acid) } & Infection & 0.011 & 1 & 0.011 & 5.36 & $\mathbf{0 . 0 2 7 1}$ \\
\hline & Tissue & 0.00 & 1 & 0.00 & 0.11 & 0.7378 \\
\hline & Interaction & $1.11 \mathrm{e}-5$ & 1 & $1.11 \mathrm{e}-5$ & 0.01 & 0.9428 \\
\hline \multirow{3}{*}{$22: 6 n-3$ (DHA) } & Infection & 0.00 & 1 & 0.00 & 0.010 & 0.023 \\
\hline & Tissue & 0.086 & 1 & 0.086 & 5.744 & 0919 \\
\hline & Interaction & 0.001 & 1 & 0.001 & 0.051 & 0.824 \\
\hline \multirow{3}{*}{ Sum SFA } & Infection & 0.038 & 1 & 0.038 & 6.14 & 0.019 \\
\hline & Tissue & $3.58 \mathrm{e}-5$ & 1 & $3.58 \mathrm{e}-5$ & 0.006 & 0.940 \\
\hline & Interaction & 0.006 & 1 & 0.006 & 0.982 & 0.329 \\
\hline \multirow{3}{*}{ Sum C18 PUFA } & Infection & 0.029 & 1 & 0.029 & 0.637 & 0.431 \\
\hline & Tissue & 0.263 & 1 & 0.263 & 5.85 & 0.021 \\
\hline & Interaction & 0.031 & 1 & 0.031 & 0.680 & 0.416 \\
\hline \multirow{3}{*}{$\begin{array}{c}\text { Average lipid/sample (\% } \\
\text { DW) }\end{array}$} & Infection & 546.607 & 1 & 546.607 & 17.684 & 0.0001 \\
\hline & Tissue & 64.550 & 1 & 64.550 & 2.088 & 0.158 \\
\hline & Interaction & 0.79 & 1 & 0.79 & 0.003 & 0.960 \\
\hline
\end{tabular}

*Significant at a level of $\mathrm{p}<0.05$. $n=9$ in all groups. 
Table 3.4. Univariate factorial ANOVA results of the effects of infection and tissue type on the absolute mass fraction ( $\mu \mathrm{g}$ of FA per mg of sample DW) composition of Helisoma trivolvis snails.

\begin{tabular}{|c|c|c|c|c|c|c|}
\hline FA Code & Source & $\begin{array}{l}\text { Type III Sum of } \\
\text { Squares }\end{array}$ & df & Mean Square & F Value & $p$-value \\
\hline \multirow{3}{*}{$16: 0$} & Infection & 17.811 & 1 & 17.811 & 1.855 & 0.183 \\
\hline & Tissue & 44.847 & 1 & 44.847 & 4.671 & 0.038 \\
\hline & Interaction & 32.137 & 1 & 32.137 & 3.347 & 0.077 \\
\hline \multirow{3}{*}{$17: \ln -7$} & Infection & 0.045 & 1 & 0.045 & 0.732 & 0.399 \\
\hline & Tissue & 0.084 & 1 & 0.084 & 1.367 & 0.251 \\
\hline & Interaction & 0.288 & 1 & 0.288 & 4.680 & 0.038 \\
\hline \multirow{3}{*}{$18: 2 n-6 c$} & Infection & 9.845 & 1 & 9.845 & 4.235 & 0.048 \\
\hline & Tissue & 4.642 & 1 & 4.642 & 1.997 & 0.167 \\
\hline & Interaction & 2.617 & 1 & 2.617 & 1.126 & 0.297 \\
\hline \multirow{3}{*}{$18: 3 n-3$} & Infection & 152.926 & 1 & 152.926 & 5.047 & 0.032 \\
\hline & Tissue & 19.260 & 1 & 19.260 & 0.636 & 0.431 \\
\hline & Interaction & 2.595 & 1 & 2.595 & 0.086 & 0.772 \\
\hline \multirow{3}{*}{$18: 3 n-6(G L A)$} & Infection & 0.045 & 1 & 0.045 & 0.728 & 0.400 \\
\hline & Tissue & 0.020 & 1 & 0.020 & 0.317 & 0.577 \\
\hline & Interaction & 0.27 & 1 & 0.27 & 4.424 & 0.043 \\
\hline \multirow{3}{*}{$22: 3 n-3$} & Infection & 0.635 & 1 & 0.635 & 4.643 & 0.039 \\
\hline & Tissue & 0.422 & 1 & 0.422 & 3.089 & 0.088 \\
\hline & Interaction & 0.014 & 1 & 0.014 & 0.099 & 0.755 \\
\hline \multirow{3}{*}{$22: 0$} & Infection & 0.005 & 1 & 0.005 & 0.076 & 0.784 \\
\hline & Tissue & 0.267 & 1 & 0.267 & 4.501 & 0.042 \\
\hline & Interaction & 0.122 & 1 & 0.122 & 2.048 & 0.162 \\
\hline \multirow{3}{*}{$20: 3 n-6$} & Infection & 4.448 & 1 & 4.448 & 0.032 & 0.051 \\
\hline & Tissue & 2.340 & 1 & 2.340 & 3.779 & 0.151 \\
\hline & Interaction & 4.892 & 1 & 4.892 & 5.175 & 0.041 \\
\hline \multirow{3}{*}{$20: 3 n-3$} & Infection & 9.402 & 1 & 9.402 & 1.857 & 0.183 \\
\hline & Tissue & 25.105 & 1 & 25.105 & 4.957 & 0.033 \\
\hline & Interaction & 0.851 & 1 & 0.851 & 0.168 & 0.685 \\
\hline \multirow{3}{*}{$20: 5 n-3$ (EPA) } & Infection & 20.561 & 1 & 20.561 & 4.817 & 0.036 \\
\hline & Tissue & 5.866 & 1 & 5.866 & 1.374 & 0.250 \\
\hline & Interaction & 4.839 & 1 & 4.839 & 1.134 & 0.295 \\
\hline \multirow{3}{*}{$22: 4 n-6$} & Infection & 0.639 & 1 & 0.639 & 7.890 & 0.008 \\
\hline & Tissue & 0.612 & 1 & 0.612 & 7.558 & 0.010 \\
\hline & Interaction & 0.462 & 1 & 0.462 & 5.701 & 0.023 \\
\hline \multirow{3}{*}{$22: 5 n-3$} & Infection & 0.070 & 1 & 0.070 & 9.096 & 0.005 \\
\hline & Tissue & 0.059 & 1 & 0.059 & 7.659 & 0.009 \\
\hline & Interaction & 0.055 & 1 & 0.055 & 7.126 & 0.012 \\
\hline \multirow{3}{*}{ 22:6n-3 (DHA) } & Infection & 0.043 & 1 & 0.043 & 9.733 & 0.004 \\
\hline & Tissue & 0.045 & 1 & 0.045 & 10.085 & 0.003 \\
\hline & Interaction & 0.044 & 1 & 0.044 & 9.781 & 0.004 \\
\hline
\end{tabular}

*Significant at a level of $\mathrm{p}<0.05$.

$n=9$ in all groups. 


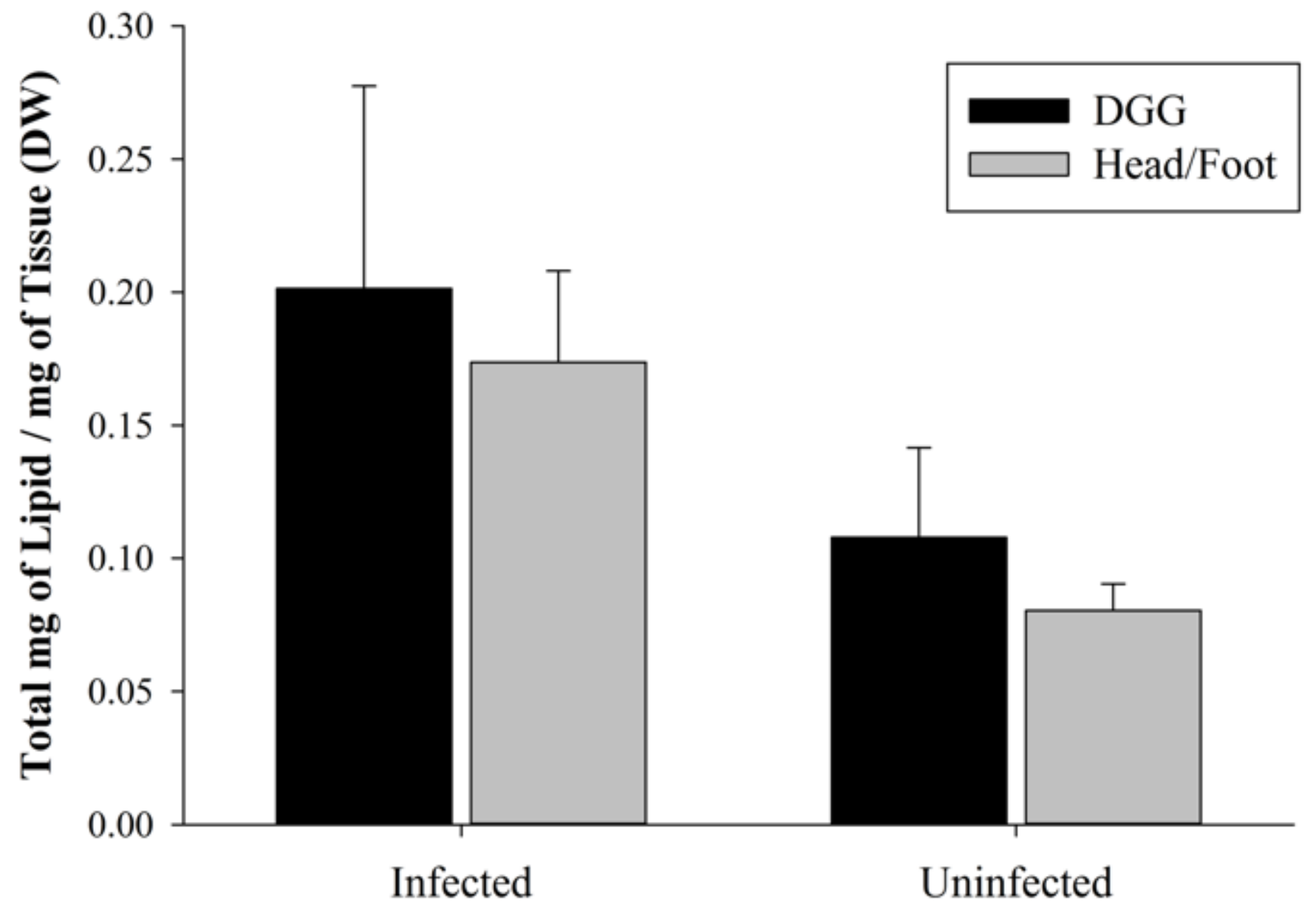

Snail Infection Status

Figure 3.1. Mean total lipid (mg of lipid/mg of tissue) ( \pm S.D.) of Ribeiroia ondatrae infected and uninfected Helisoma trivolvis snails grouped by snail infection status (DGG = digestive-gland gonad complex and Head/Foot tissue). Gravimetric results differed significantly based on infection status $(\mathrm{P}=0.0001$, Table 3.3). Error bars: $+/ 1 \mathrm{SD}$. 


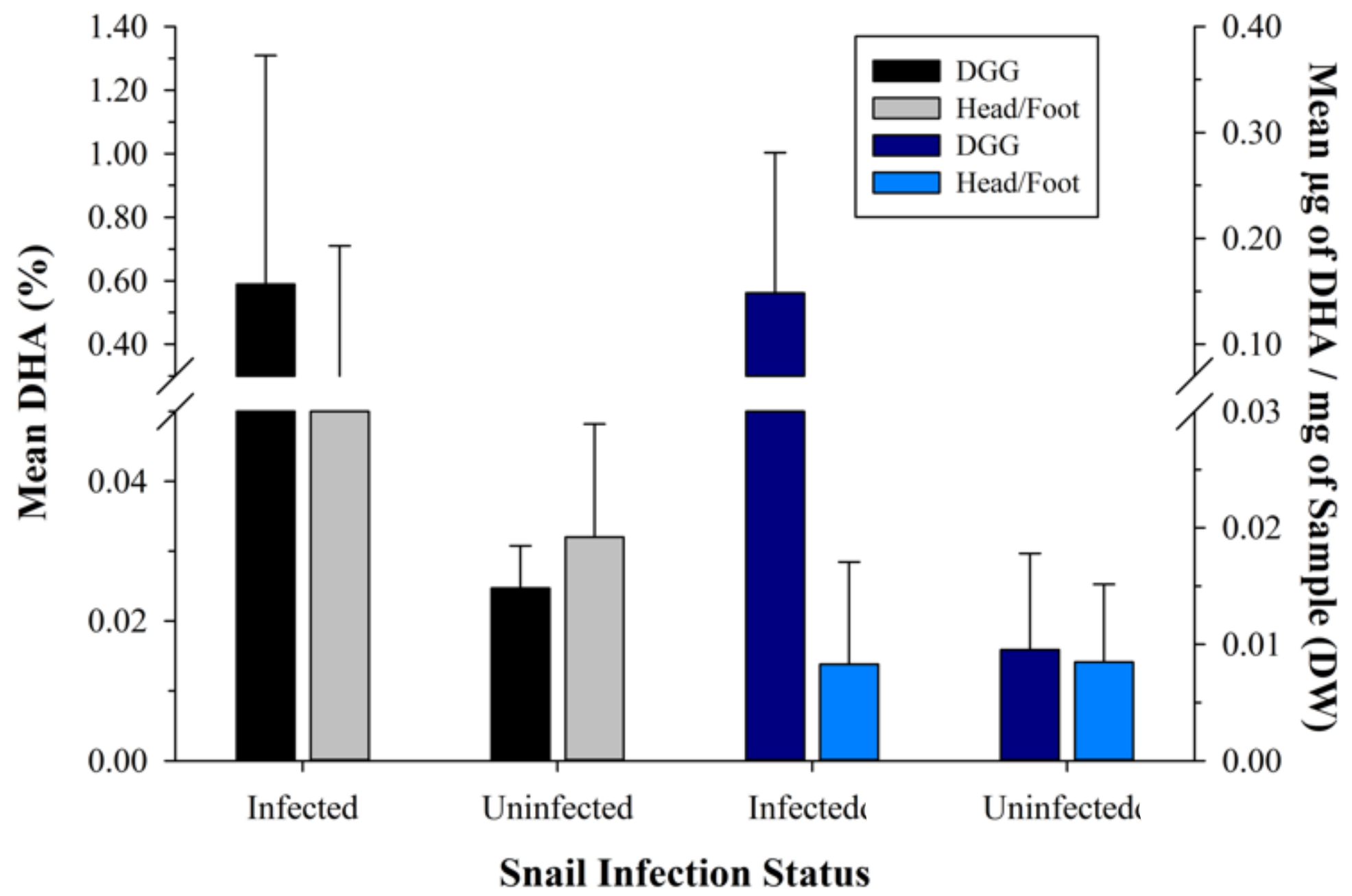

Figure 3.2. Mean \pm S.D. proportional (\%) and absolute mass fraction ( $\mu \mathrm{g}$ of FA per $\mathrm{mg}$ of sample DW) of DHA composition of Ribeiroia ondatrae infected and uninfected Helisoma trivolvis snails grouped by snail infection status ( $\mathrm{DGG}=$ digestive gland-gonad complex and Head/Foot tissue). Gravimetric and proportional results differed based on an interaction with the infection status and tissue type and infection status respectively $(\mathrm{P}=0.0004, \mathrm{P}=0.023$, Table $3.4,3.3)$. 


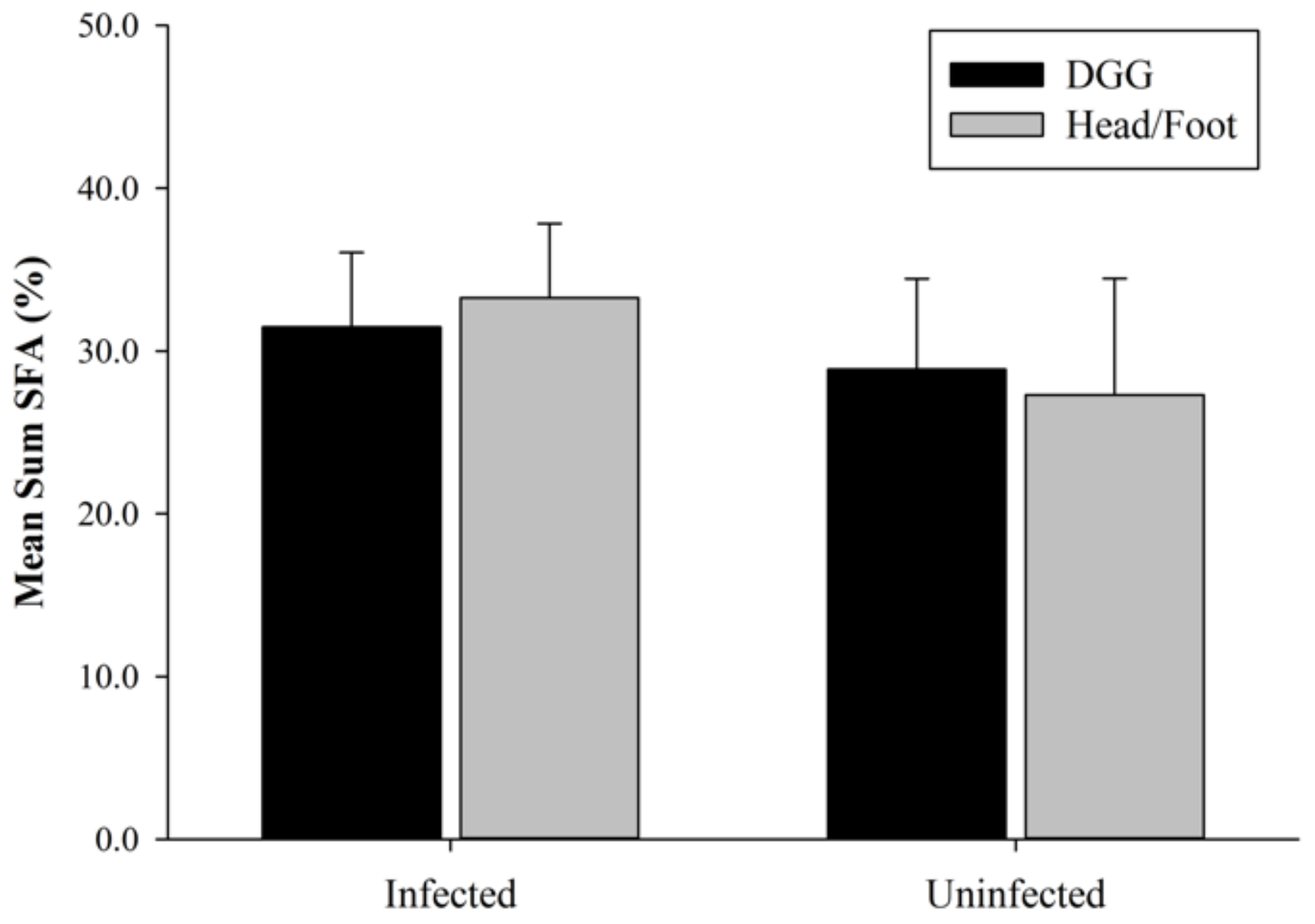

Snail Infection Status

Figure 3.3. Mean ( \pm S.D.) proportional SFA composition (\%) of Ribeiroia ondatrae infected and uninfected Helisoma trivolvis snails grouped by snail infection status ( $\mathrm{DGG}=$ digestive glandgonad complex, Head/Foot = head/foot tissue). Gravimetric results differed significantly based on infection status $(\mathrm{P}=0.019$, Table 3.3$)$. 


\section{Discussion}

I found that trematode infection affected the lipid composition of the host snail, but that specific effects on lipid composition differed by tissue type, and some interactive effects were also seen. Here I provide the first report on the FA composition of the Ribeiroia ondatraeHelisoma trivolvis trematode-host relationship. Tissue (DGG or head/foot) from infected snails contained significantly more total lipids (mg of lipid/mg of tissue) as well as higher proportions of myristic (14:0), oleic (18:1n-9t), nervonic (24:1n-9), adrenic (22:2n-6), DHA and SFA acids than the same tissues from uninfected controls (Fig. 3.3). There were also tissue differences irrespective of host infection status, with DGG complexes containing higher proportions of nonadecylic (19:0), 20:1n-12, 20:3n-3 and head/foot tissue containing higher proportions of ARA. Additionally, infected $H$. trivolvis snails had significantly higher mass fractions ( $\mu \mathrm{g}$ of FA/mg sample DW) than uninfected tissue for 18:2n-6, 18:3n-3, 22:3n-3 and 20:5n-3. Infected H. trivolvis snail bodies had less 18:3n-6 (GLA) than uninfected body tissue, and infected head/foot tissue had higher quantities than uninfected head/foot. Infected body tissue had the highest mass fraction of 22:6n-3 (DHA) than infected foot, uninfected body and uninfected head/foot. The observed increase in the lipid content of infected hosts suggests that trematode infections have patho-biochemical effects on the lipid composition of their hosts or are perhaps driven by lipid secretions from the parasites (Southgate 1970; Lunetta and Vernberg 1971; Fried et al. 1993a, b; Fried et al. 1998c). The role(s) of lipids in larval trematode parasites (rediae and cercariae) are still somewhat speculative, and therefore further investigations into the mechanisms and role of lipids in the parasite-host relationship, as well as within parasites themselves, are required. The results of this study contribute to that body of literature by confirming that trematode infection can induce unique changes in snail host composition (and see Lunetta and Vernberg 1971), and that this may therefore have trophic-level implications related to nutrient movement.

Lunetta and Vernberg (1971) investigated the FA composition of Ilyanassa obsoleta snails infected with different digeneans and observed unique differences in host FA composition based on trematode type, and they also noted some broad patterns that were confirmed by later studies with other snail and trematode species (Fried et al. 1993b). For instance, observed increases in neutral lipids of infected snails by Lunetta and Vernberg (1971) are also consistent with reports by Fried and Sherma (1990) working with the H. trivolvis/E. trivolvis host-parasite relationship, 
as well as Muller et al. (2000) and Thompson (1987), studying B. glabrata/Schistosoma mansoni (Sambon, 1907). However, others have reported decreases in neutral lipids within infected snails, such as Fried et al. (1989) working on E. caproni in B. glabrata, and McManus et al. (1975) working on the trematode Microphallus similis in the snail Littorina saxatillis rudis. The variation observed in different parasite-host relationships suggests that certain species of larval trematode may directly or indirectly induce changes in host lipid composition (Fried et al. 1993b).

Here, the increase in total lipids within $H$. trivolvis due to infection with $R$. ondatrae is in accord with the increases in neutral lipids previously reported for snails (H. trivolvis) infected with E. trivolvis and S. mansoni, and suggests that trematodes increase neutral lipid depots in hosts, either through secretion of lipids by the parasite or via an infection-driven change in host metabolism (Fried et al. 1998). Tunholi-Alves et al. (2011) observed changes in the lipid composition of snail hosts through time in B. glabrata infected with E. paraensei. Lipid composition decreased after the parasite infection was first established and subsequently increased over time (Tunholi-Alves et al. 2011). It was postulated by Tunholi-Alves et al. (2011) that TAG is used as an energy source by both the snail and parasites, as enzymes related to lipid metabolism, specifically non-specific lipases and esterases, have been identified in trematodes (Erasmus 1972). The increase in the total lipids of infected snails can therefore perhaps be explained by the mobilization of neutral lipids, which are stored as TAG in the snail bodies and serve as a precursor in mitochondrial oxidation to form ATP, supplying energy to both the parasite and host (Klobučar et al. 1997; Tunholi-Alves et al. 2011). It has also been suggested that the Phylum Platyhelminthes may possess the ability to synthesize FA de novo as they are closely related to the taxon (Rotifera) which was demonstrated to contain the $121 \omega x$ desaturase sequence that is necessary to synthesize n-3 PUFA (Edgecombe et al. 2011; Kabeya et al. 2018).

Furthermore, behavioral changes within the snail host may also account for increase snail host lipid content. It is possible that infected hosts were engaging in compensatory eating, and therefore consuming more algae leading to increased lipid profiles within the infected snails (Bernot and Lamberti 2008). Additionally, organisms under stress conditions will preferentially accumulate EFA as a mechanism to ensure survival and will therefore preferentially metabolize less important FA, as a means to conserve EFA (Tocher 2013). 
Fried et al. (1993a) reported a marked decrease in the proportional levels of saturated fatty acids (SFA) in the bodies of infected snails, and increased levels in the DGG complex of the whole body as well as the DGG complex of B. glabrata snails infected with E. caproni rediae. Considering my results, $R$. ondatrae parasites in $H$. trivolvis induced a similar significant increase in the proportion of SFA in the whole bodies of infected snails. However, without a direct comparison of snail type and trematode type, concluding whether differences were driven by the trematode infection, snail type, or a combination is difficult. Fried et al. (1993b) investigated the effect of snail type on patho-biochemistry by using $H$. trivolvis snails infected with the same larval trematode (E. caproni) as the snail species (B. glabrata) examined in a previous study (Fried et al. 1993a). Further to this contrast, they also investigated the DGG complex of uninfected snails, rediae freed from the DGG complex, uninfected DGG, as well as adult echinostome worms. The DGG of infected snails had higher levels of stearic acid (18:0), hexatrienoic acid (16:3n-4), and DHA compared to uninfected controls. The DGG of uninfected snails exhibited higher levels of 20:2 NMID and adrenic acid than infected snails. I also investigated $H$. trivolvis snails, but infected by $R$. ondatrae, and observed an increase in the proportions of myristic, adrenic, nervonic and oleic acids, as well as increased DHA (both proportionally and mass fraction). Uninfected $H$. trivolvis snails used in Fried et al. (1993a) were similar in proportional composition to $H$. trivolvis snails used in this study (SFA: 33 vs. 31.5; MUFA: 25 vs. 23.5; PUFA: 40 vs. 45.0) respectively. Given that $H$. trivolvis snails infected with $R$. ondatrae and E. caproni differed in their FA composition post infection, suggests that different trematode species have distinct influences in this context.

Interestingly, based on my findings and previous reports, $R$. ondatrae and E. trivolvis parasites both increased the proportion and absolute mass fraction of DHA in snail host tissue. DHA is an LC-PUFA which has important functions in organism health, as well as development of neural and ocular tissue (Arts et al. 2001; Innis 2007; Farooqui 2009). Aquatic ecosystems serve as the main source of DHA, through which higher trophic level organisms achieve their dietary needs for optimal function (Kainz et al. 2004; Hixson et al. 2015). Observing an increase in snail tissue DHA due to trematode infection may thus have important implications in the trophic transfer of this essential biomolecule. Elevated quantities of DHA in snail tissue due to trematode infection may provide an additional source of DHA for higher trophic level consumers, as well as the newly identified potential for cercariae to serve as a source themselves. 
In Chapter 2, I quantified the FA composition of $R$. ondatrae cercariae. The same FA that were higher in infected snail tissue here were also estimated, on a per cercaria basis in Chapter 2 (Table 2.2), as the following: $2.81 \mathrm{ng} 14: 0 ; 0.17 \mathrm{ng} 22: 2 \mathrm{n}-6 ; 0.18 \mathrm{ng} 24: 1 \mathrm{n}-9 ; 0.19 \mathrm{ng}$ 18:1n-9; $6.566 \mathrm{ng}$ SFA; and $0.005 \mathrm{ng}$ DHA. Interestingly, other than DHA, these FA were not the most abundant on an absolute basis within $R$. ondatrae cercariae, suggesting that other factors, such as host metabolism, contribute to lipid composition of rediae and snail host tissue.

In an attempt to further tease apart the drivers of changes in host lipids, Fried et al. (2001) investigated the effect of snail diet on DGG composition. They observed that lipid-rich diets induced changes in the levels of free sterols, but not TAG, in the DGG of B. glabrata snails infected with S. mansoni. These results, coupled with observations of distinct differences in lipid composition based on parasite-host type, suggest that lipid levels depend on numerous factors, such as diet, parasite species, host species, and environmental conditions (Fried et al. 2001). Another suggested driver of snail tissue lipid levels was proposed by Bandstra et al. (2006), who postulated that infection strength may affect host lipid levels, as miracidial dose has been observed to affect the reproductive and metabolic activities of infected snails (Massoud 1974, Théron et al. 1992, Tunholi-Alves et al. 2011).

However, there are still many unexplored possibilities regarding the typically elevated levels of lipids in trematode-infected snails. A recent study identified the presence of the $121 \mathrm{\omega x}$ desaturase sequence that is necessary to synthesize n-3 PUFA de novo (Kabeya et al. 2018) in the Phylum Mollusca, in which the class Gastropoda belongs (Kabeya et al. 2018). The potential for gastropods to produce FA highlights their ability to modify FA composition regardless of previously identified factors (i.e. diet or trematode infection as per Fried et al. 1992b). Infected H. trivolvis DGG tissue contained higher proportions (infected higher than uninfected whole bodies) and absolute mass fractions (higher in infected DGG than infected head/foot, uninfected head/foot and DGG) of DHA. The observation of increased DHA may be due to de novo synthesis from parasites themselves, or perhaps a response from the snail host to increase DHA production in response to the tissue damage experienced from the trematode infection.

Irrespective of infection, H. trivolvis DGG and head/foot tissue differed significantly in FA composition, with DGG tissue containing higher proportions of nonadecylic (19:0), 20:1n-12, 20:3n-3 and oleic (18:1n-9t) acids, and head/foot tissue containing higher proportions of ARA. High proportions of ARA, oleic, as well as isomers of oleic (18:1) and eicosanoic (20:1) acids 
are present in other freshwater gastropods (Gonoiobasis virginica, Physa sp., Viviparous malleatus, B. glabrata) and therefore it was not unusual to identify high proportions in $H$. trivolvis tissue (Dembitsky et al. 1992; Fried et al. 1992a,b; Fried et al. 1993a,b,c). Alpha ( $\alpha-$ ) linoleic and $\gamma$-linolenic acid (i.e. 18:3n-3 and 18:3n-6, respectively) have also previously been identified to comprise minor fractions overall in the $H$. trivolvis snails, but were found to be the most abundant trienes (Fried et al. 1993b). Here, 18:3n-3 had the highest proportion of the trienes, with eicosatrienoic acid (20:3n-3) comprising the second highest triene in $H$. trivolvis tissue.

The results of my study, coupled with previous findings, confirm that larval trematode species induce unique changes to snail host lipids. However, further investigations into FA synthesis within gastropods and parasites, as well as the influence of diet, snail type and environmental conditions on host composition are needed to further illuminate key patterns. Trematode parasites affect the energy reserve lipids, growth and behaviour of their gastropod hosts, which affects the abundance of parasites themselves, as well as the survival of the intermediate hosts (Lunetta and Vernberg 1971; Sousa 1983; Levri and Lively 1996; Sorensen and Minchella 1998). These life history changes can also affect the distribution and abundance of their final hosts, some of which are a concern for population conservation (Johnson et al. 2002). As snails commonly serve as key grazers in aquatic ecosystems, differences in their habitat use due to infection, can also induce changes to the standing crop and spatial distribution of algae (Bernot and Turner 2001; Bernot 2003). Because certain algal taxa serve as key sources of FA, some of which are limiting in aquatic ecosystems (Müller -Navarra 1995; see Chapter 1 for role of algae in essential fatty acid transfer), changes in algal abundance alters nutrient flow in aquatic food webs. Additionally, an alteration in snail composition due to trematode infection may affect their ability to serve as nutrient rich food for the predators that consume them.

Further, as climate change induces shifts in freshwater habitats (i.e. higher temperatures), these will be experienced by snails which could affect their lipid profiles through altered food sources or heat stress (Johnson et al. 2002). Additionally, higher temperatures facilitate an increase in cercarial production (e.g., Johnson et al. 2002; Poulin 2006). If the lipid profiles of the snail change, that may in turn affect lipid profiles of cercariae, possibly altering their quality as a source of EFA for aquatic consumers. Not only is cercariae production sensitive to increased temperatures, climate warming may also cause trematodes to become active earlier in the season, 
as optimal development temperatures are reached earlier (Poulin 2006; Koprivnikar and Poulin 2009; Paull and Johnson 2011; Paull et al. 2015). Marked increases or decreases in cercarial emergence may significantly alter their biomass within aquatic systems, either through increased or decreased nutrient contribution. Cercariae serve as prey for aquatic predators (i.e. dragonflies), and higher temperatures induce the early emergence of dragonfly larvae (Leucorrhinia intacta), these changes may allow for longer ( $R$. ondatrae) or shorter seasons of cercariae consumption, if cercariae species experience thermal stress (Poulin 2006; Paull et al. 2015). Understanding the response of parasites to temperature, in relation to their nutritional value as a prey item, will allow for a more robust understanding of the contribution of cercariae to food web dynamics. 


\title{
CHAPTER 4: RIBEIROIA ONDATRAE CERCARIAE AS AN ESSENTIAL FATTY ACID-RICH FOOD SOURCE FOR HIGHER TROPHIC LEVEL CONSUMERS
}

\author{
Keira M. McKee, Janet Koprivnikar, Michael T. Arts \\ Parts of this chapter will be submitted as a section of Chapter 2 to Oecologica.
}

\begin{abstract}
Recent evidence suggests that free-living infectious stages of parasites contribute substantially to the biomass of aquatic ecosystems, also serving as prey for higher trophic levels. The motile cercariae of trematode parasites are similar in size and abundance to zooplankton, both of which are preyed upon by a range of freshwater predatory invertebrates and vertebrates. Zooplankton are a vital source of energy and nutrients, and are an especially important link for the movement of essential lipid biomolecules from algae to higher trophic levels, a process which regulates aquatic ecosystem productivity. Cercariae have not previously been considered as a significant prey item however increasingly they are being shown tocontribute substantially to the biomass in aquatic systems. My study compared cercariae (Ribeiroia ondatrae) and zooplankton (Daphnia sp.) as prey items for dragonfly larvae (Leucorrhinia intacta), a top predator in freshwater systems. Forty larval dragonflies were collected, measured (head width) and maintained in the lab in separate containers. Dragonfly larvae were fed wet mass equivalents of either Daphnia or $R$. ondatrae cercariae for a total of 5 weeks, after which they were re-measured for head width and then used for fatty acid (FA) analysis. I found no significant difference in the growth or essential fatty acid (EFA) profiles of dragonfly larvae fed either R. ondatrae or D. pulex. This suggests that trematode cercariae are an equally nutritionally viable food source relative to zooplankton, supporting predator growth and contributing to ecosystem productivity through the trophic transfer of EFA. The identification of novel sources of EFA is vital as they represent a primary energy source in aquatic ecosystems and are necessary for vertebrate health and survival. Further studies are needed to investigate the specific fatty acid profile of Ribeiroia ondatrae as well as determine its digestibility and the assimilation of nutrients into predator tissue.
\end{abstract}




\section{Introduction}

It is well known that parasites, especially free-living infectious stages, constitute significant biomass in aquatic ecosystems (Kuris et al. 2008; Kaplan et al. 2009; Preston et al. 2013; Soldánová et al. 2016). However, their ability to serve as prey items for organisms at higher trophic levels is poorly understood. Recently, the standing stock biomass of parasites living in an estuarine food web in California were found to be comparable to the biomass of several other major groups of free-living aquatic animals in these systems and larger than that of the principal top predators (Kuris et al. 2008).

Cercariae, a free-swimming phase of the trematode parasite life cycle, are produced in high numbers and readily consumed by a variety of aquatic invertebrates and vertebrates (Morley 2012; Orlofske et al. 2012, 2015; Thieltges et al. 2013; Catania et al. 2016). Although consumption of cercariae by predators has been established, the contribution of cercariae (as a prey item) to the overall flow of nutrients in aquatic food webs is less understood (Johnson et al. 2010). In fact, cercariae have not typically been considered as members of the aquatic zooplankton community, and are thus rarely, if ever, included as prey items in aquatic food web models (e.g., Marcogliese and Cone 1997), even though they may play a similar role as other aquatic prey (Morley 2012). However, studies highlighting their abundance and ability to affect aquatic food webs including trophic interactions, linkage strength and food-web structure dynamics, have, more recently, garnered support for their inclusion in discussions of nutrient flows within aquatic ecosystems (Marcogliese and Cone 1997; Lafferty et al. 2006; Lafferty et al. 2008; Byers 2009; and reviewed by Sukhdeo 2012).

Cercariae range in size from 0.2 to $0.9 \mu \mathrm{m}$ (depending on the trematode species) and are analogous to meroplankton (planktonic larvae of benthic organisms), and, more importantly, overlap in size with many zooplankton and zoobenthos species (Morley et al. 2012). Because of their similarity in size, cercariae and zooplankton both have the potential to serve as prey items for a variety of aquatic invertebrates such as larval insects, oligochaetes, and copepods, as well as vertebrates such as larval fish (Cort 1914; Schotthoefer et al. 2007; Kaplan et al. 2009; Orlofske et al. 2012, 2015; Catania et al. 2016).

Investigations into the biochemical composition of cercariae have determined that they are rich in neutral lipids and glycogen, suggesting that they are nutritious prey items (Moore and Halton 1975; Diconza and Basch 1976; Fried et al. 1998a; Furlong et al. 1992; Nanduri et al. 
1991; Xu et al. 1994; Marsit et al. 2000a,b; Schariter et al. 2002; Thieltges et al. 2008). These efforts have primarily employed the use of Oil Red-O (ORO) and Nile Blue Sulphate as means to stain and visually detect the presence of neutral lipids (primarily triacylglycerides) in rediae, cercariae and sporocysts of various trematode species (Moore and Halton 1975; Furlong et al. 1995; Ginetskinskaya 1988; Fried et al. 1998b; Marsit et al. 2000a,b). Quantitative efforts have utilized high performance thin-layer chromatography (HPLC) to conclude that the majority of Bodipy- $\mathrm{C}_{12}$ label, a fluorescent $\mathrm{FA}$ analog, was found in neutral lipids that were concentrated in the ducts and the cercarial tail, however they did not specifically identify the FA present (Furlong et al. 1995; Marsit et al. 2000a,b; Schariter et al. 2002). As discussed in Chapter 2, Ribeiroia ondatrae cercariae contain significant quantities of long-chain polyunsaturated fatty acids (LC-PUFA), which include the essential fatty acids (EFA) EPA and DHA, and that are known to be highly bioactive molecules. Additionally, Xu et al. (1994) and Nanduri et al. (1991) also identified that the cercarial covering, termed the glycolax, contains fucose, galactose, amino sugars and glucose (Nanduri et al. 1991; Xu et al. 1994). Finally, cercariae tails have significant glycogen stores to supply them with energy for survival as they are non-feeding (Xu et al. 1994). These studies suggest that the cercariae of multiple species of trematode parasites are lipid- and glycogen-rich, as well as contain essential bioactive lipids.

Bioactive lipids are vital for the health of all aquatic and terrestrial organisms as they critically influence physiology, have fundamental roles in cell membrane function, serve as precursors for eicosanoids, act as essential signalling molecules for reproduction and promote neural and ocular development (Stillwell and Wassall 2003; Stanley 2006; Hoffman et al. 2009; Scholtz et al. 2016). These lipids are conserved/retained and transferred in food webs, species that lack either the preformed long-chain lipids or the metabolic machinery to make an essential component, are physiologically negatively affected (Lands 2009). The long-chain preformed bioactive lipids are: arachidonic acid (ARA; 20:4n-6), EPA and DHA. However, bioactive lipids also include the precursors $\alpha$-linolenic acid (ALA; 18:3n-3) and linoleic acid (LNA; 18:2n-6), which through a series of metabolic steps, are converted to their n-3 or n-6 LC-PUFA that are physiologically vital.

The n-3 LC-PUFA, EPA and DHA have important roles in the modulation and prevention of human diseases. EPA is vital in the healthy functioning of the cardiovascular system in humans and DHA is important for proper brain and retina development and contributes to cardiovascular 
health (Lauritzen et al. 2001; Kris-Etherton et al. 2002; SanGiovanni and Chew 2005; McNamara and Carlson 2006; Plourde and Cunnane 2007; Bazan 2009; Wall et al. 2010; Phang et al. 2011). ALA and LNA are considered essential since most animals are unable to insert the n-3 or n- 6 double bond into a FA. ALA and LNA, the n-3 and n-6 respective precursors, are converted to EPA and DHA (n-3) or ARA (n-6) through a series of modifications and therefore is considered to be biologically active. The n-6 FA, ARA, has significant benefits in reducing thrombosis, a major complication in coronary atherosclerosis, which leads to myocardial infarction (Connor 2000).

Zooplankton are recognized as high-quality prey and are therefore a crucial link for the trophic transfer of essential nutrients (including bioactive lipids) from the base of aquatic food webs to higher trophic level organisms (Müller-Navara 1995). The FA composition of zooplankton is influenced by the FA composition of their diets, as well as the ability of some species to selectively retain and metabolically alter dietary EFA, leading to variable FA profiles, but which are nonetheless generally conserved when comparing predator to prey (Weers et al. 1997; Von Elert 2002; Brett et al 2006a, b; Graeve et al. 2005; Veloza et al. 2006; Smyntek et al. 2008; Ravet et al. 2010). Additionally, they function to trophically transfer LC-PUFA (see Chapter 1), as well as shorter chain ALA and LNA. Long-chain polyunsaturated FA ( $>20 \mathrm{C}$ atoms) are primarily synthesized at the base of aquatic food webs by primary producers (protists) such as diatoms, cryptophytes, euglenoids and dinoflagellates (Brett and Müller-Navarra 1997; Kainz et al. 2004; Arts et al. 2009; Gladyshev et al. 2013) and trophically retained in higher organisms.

As aquatic ecosystems provide the primary source of pre-formed LC-PUFA, it is essential to understand the various pathways for trophic transfer of these biologically-active molecules from the base of the aquatic food web to higher trophic level organisms both inside aquatic ecosystems and in adjacent terrestrial areas (which may receive these EFA through a variety of pathways e.g., insect emergence). LC-PUFA are produced in abundance in specific algal taxa in aquatic ecosystems; however, the bioavailability of LC-PUFA to consumers is not only dependent on the taxonomic composition of the producers, but also on that of the zooplankton (Sushchik et al. 2004; Persson and Vrede 2006; Burns et al. 2011; Galloway and Winder, 2015; Strandberg et al. 2015). Zooplankton and zoobenthos consume LC-PUFA-rich algae, serving as the primary bridge for the transfer of these essential biomolecules across the plant-animal 
interface (Müller-Navarra 1995; Gulati and DeMott 1997; Danielsdottir et al. 2007). Particularly important is the transfer of EPA and DHA from algae to higher trophic levels, as they are vital to the somatic growth and reproduction of herbivorous zooplankton, and a key driver in aquatic ecosystem productivity (Müller-Navarra 1995, 2000; Ravet et al. 2003). Critically, carnivorous organisms such as larval odonates and fish consume LC-PUFA rich zooplankton, and, in the process, selectively retain and subsequently transfer these essential nutrients to progressively higher trophic levels (Kainz et al. 2004; Arts et al. 2009; Gladyshev et al. 2009).

Aquatic gastropods such as snails serve as the first intermediate hosts in which asexual reproduction of parasites take place. Cercariae develop in sporocysts or rediae, in which the former absorbs host-derived nutrients, while the latter consumes host tissue with a mouthpart. Importantly, many trematode species utilize snails whose diet consists primarily of algae, some of which contain significant quantities of EPA and DHA (Lombardo et al. 2002). Although trematode cercariae do not feed, their EFA profiles may be similar to that of zooplankton which consume primary producers. Like zooplankton, cercariae serve as prey items for a variety of aquatic organisms and therefore also have the potential to accumulate and trophically transfer LC-PUFA that is derived from the tissue of their algivore host to higher trophic levels.

Aquatic ecosystems provide dietary LC-PUFA for aquatic and terrestrial vertebrates (Gladyshev et al. 2009). As such, the connectivity between aquatic and terrestrial ecosystems is critical, particularly the transfer of nutrient subsidies from wetland organisms to terrestrial organisms that live in and around those habitats (Hixson et al. 2015). There are multiple means through which LC-PUFA are transferred to the surrounding terrestrial landscape. Most evident is the direct consumption of aquatic LC-PUFA rich organisms such as fish by terrestrial piscivores, including herons, eagles, osprey, otters, bears, etc. (Gladyshev et al. 2009, 2013; Hixson et al. 2015). Another vital, mechanism is the transfer of freshwater-derived LC-PUFA through emergent aquatic insects (Gladyshev et al. 2009).

Aquatic insects, including predatory larval odonates such as the dragonfly Leucorrhinia intacta, accumulate LC-PUFA through their diet (Walker and Corbet 1975; Martin-Creuzburg et al. 2017). The larvae of this species mature over a ten-month period before emerging onto the terrestrial landscape in their adult form, bringing with them their aquatically-derived lipid subsidies (Wissinger, 1989). Although larval odonates consume a variety of prey, L. intacta are generalist predators that attack prey within a specific size range (Catania et al. 2016), including 
the following: zooplankton, zyopteran larvae, dipteran larvae, and other dragonfly larvae species (Pritchard 1964). Recent studies have shown that L. intacta also readily consume trematode cercariae of multiple species including Cotylurus sp., Posthodiplostomum sp. and Ribeiroia ondatrae; even when Daphnia pulex are offered as an alternative prey item (Schotthoeffer et al. 2007; Orlofske et al. 2012, 2015; Catania et al. 2016). Although it has been established that $L$. intacta are voracious predators of trematode cercariae, the potential for cercariae to serve as a trophic link for the transfer of LC-PUFA from aquatic to terrestrial ecosystems through emergent aquatic insects has yet to be explored. It is also unclear whether trematode cercariae represent a nutritionally comparable food source, relative to zooplankton, for consumers.

I investigated the growth, total lipid, and FA composition of L. intacta larvae fed equivalent masses of either trematode cercariae ( $R$. ondatrae) or freshwater zooplankton (Daphnia spp.) to assess the extent to which cercariae served as a nutrient-rich food source by comparing the growth of $L$. intacta fed the two different diets. In addition, I also investigated the potential for cercariae to transfer lipid subsidies from the base of the aquatic food web to higher trophic levels in both aquatic and, by extension, terrestrial landscapes. I hypothesized, due to the similarities in trophic levels, size and potential lipid composition of Daphnia spp. and R. ondatrae, that the dragonfly larvae fed $R$. ondatrae would show similar growth, LC-PUFA, ALA and LNA profiles to those fed the Daphnia spp. In documenting this, I uncover a novel and substantial pathway through which ALA, LNA and LC-PUFA are transferred to higher trophic level organisms in both aquatic and terrestrial ecosystems.

\section{Materials and Methods}

\section{Predator and Prey Collection}

Ribeiroia ondatrae cercariae were collected from freshwater California ponds and maintained for the feeding experiment based on the protocol described in Chapter 2 (see - Field Collection and Animal Care). Zooplankton prey (Daphnia spp.) and their freshwater consumer, dragonfly larvae of the species Leucorrhinia intacta (Hagen 1861), were collected using dip nets from the Kofler Science Reserve in King City, Canada (4357' 122 26.0352" N, 79³0' 37.1916" W) and transported to Ryerson University, Canada. Daphnia were a mix of two species, but predominantly contained Daphnia pulex, hereafter referred to as Daphnia spp. Dragonfly larvae 
were maintained in $13.2 \mathrm{~L}$ containers containing dechlorinated water overnight and were set-up for experimental use the next day. Daphnia spp. that served as prey were maintained in $13.2 \mathrm{~L}$ tubs of dechlorinated water for the duration of the experiment. They were exposed to natural light (13:11 hour light:dark cycle) and were provided with the green algae Chlamydomonas sp. (Merlan Scientific) as a food source. Importantly, the $R$. ondatrae infected snails were fed using the same algal master culture thus ensuring that the EFA produced by this algal species was available to both Daphnia spp. and the snails. Daphnia spp. acquired EFA through direct consumption, and cercariae through feeding on the snails that in turn ate this alga.

\section{Experimental Design}

The experiment consisted of two different diet treatments whereby the larval dragonflies received equivalent masses of their prey items ( $R$. ondatrae cercariae or Daphnia spp.). Each treatment contained 19 replicate $1 \mathrm{~L}$ plastic containers that each held the following: 1 dragonfly larva, plastic vegetation, and $\sim 800 \mathrm{~mL}$ of dechlorinated water. Based on previously-published information regarding the mass of the two prey items (see Table 4.1), the two food treatments consisted of either 126 R. ondatrae cercariae or 21 Daphnia spp.fed twice per week to each odonate. The rationale for the number of Daphnia spp. was to ensure that the larvae were feeding ad libitum, as larval odonates of the size I selected consume $5-7$ Daphnia daily, so $\sim 35-49$ perweek (McCauley 2008). Therefore, the number of $R$. ondatrae cercariae was chosen to be equivalent to the daily consumption of a typical L. intacta larva in nature. To ensure that $D$. pulex were of the appropriate size, I sieved the Daphnia spp. through two different mesh sizes to retain a specific size range, of $0.75-1 \mathrm{~mm}$, which corresponds to a known mass of Daphnia spp. (Table 4.1). I randomized the placement of the 38 containers to control for any variation in lab conditions; all containers were placed on plastic shelves on two separate side-by-side racks in a lab (Fig. 4.1b). The containers were maintained by replacing any evaporated water weekly with dechlorinated tap water and placed under lamps on a 14:10 light dark cycle to mimic midsummer daylength hours and optimal dragonfly larvae growth conditions. 
Table 4.1: Individual mass of each prey type (Ribeiroia ondatrae or Daphnia spp.) and their equivalence in number of individuals.

\begin{tabular}{|c|c|c|}
\hline Prey Type & Dry Mass of 1 Specimen & $\begin{array}{c}\text { Mass and Number of } \\
\text { Individuals }\end{array}$ \\
\hline R. ondatrae & $\begin{array}{c}0.880 \mu \mathrm{g} \text { (Lambden and } \\
\text { Johnson 2013) }\end{array}$ & $\begin{array}{c}126 \text { R. } \text { ondatrae }=110.88 \mu \mathrm{g} \\
21 \text { Daphnia } \text { spp. }=109.2 \mu \mathrm{g}\end{array}$ \\
\cline { 1 - 2 } $\begin{array}{c}\text { Daphnia } \text { spp. (size range of } \\
0.75 \mathrm{~mm} \text { to } 1 \mathrm{~mm})\end{array}$ & $5.2 \mu \mathrm{g}$ (Dumont et al. 1975) & \\
\hline
\end{tabular}
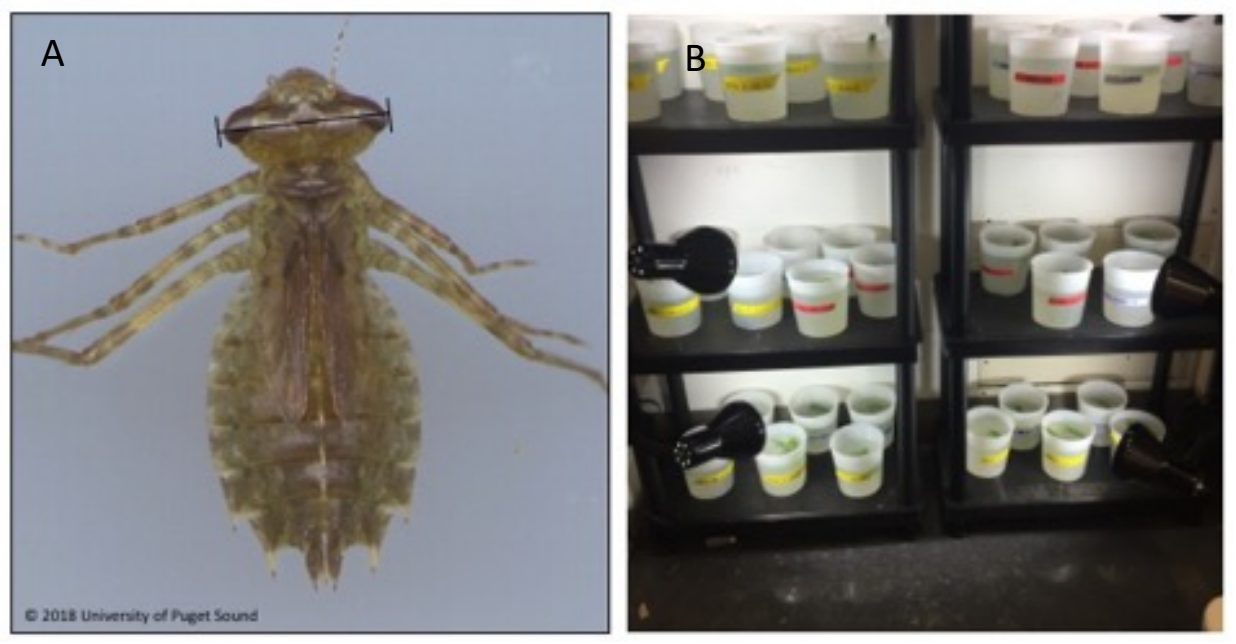

Figure 4.1. A. Leucorrhinia intacta larvae with head width measurement. B. Experimental set-up for feeding experiment, containers were randomly assigned to control for lab conditions.

\section{Experimental Procedure}

Head widths are a standard measure of larval odonate body size (e.g., Catania et al. 2017). Thus, head widths (measured using digital calipers) of the collected dragonfly larvae were measured, and those between 4-6 mm in size were kept for experimental use (Fig. 4.1a). This size range was chosen since L. intacta of this size have been previously found to prey on cercariae and D. pulex of similar sizes, including cercariae that were $<300 \mu \mathrm{m}$ and Daphnia $s p$. ranging from $~ 750-1500 \mu \mathrm{m}$ (Ebert 2005; Schottheofer et al. 2007; Catania et al. 2016) (Fig. 4.2). Additionally, larval odonates typically grow three to four orders of magnitude before their emergence into the adult terrestrial stage, therefore, using relatively smaller-sized odonates 
would allow me to observe the effect of diet on their growth prior to emergence (Marczak et al. 2006).

Ribeiroia ondatrae cercariae and Daphnia spp. were collected and counted (see Chapter 2 Field Collection and Animal Care) the morning of the intended feeding. Each of the dragonfly larvae were fed either 126 R. ondatrae or 21 Daphnia spp., 2-3 d apart for 5 weeks. The final head measurement for each larva was taken after 5 weeks of feeding. Two separate feeding experiment trials were conducted due to the small number of L. intacta larvae available at the beginning of the experiment. A first trial $(n=23)$ thus began in week 1 (August $7^{\text {th }}, 2017$ ) and a second trial $(\mathrm{n}=16)$ with an identical experimental setup was added in week 3 (August $21^{\text {st }}, 2017$ ) and underwent an identical feeding regime, with both ultimately running for a 5 -week feeding duration. At the end of the feeding trials, individual dragonfly larvae were weighed and placed into labeled $2.5 \mathrm{~mL}$ cryogenic vials and frozen at $-85^{\circ} \mathrm{C}$ for lipid analysis.

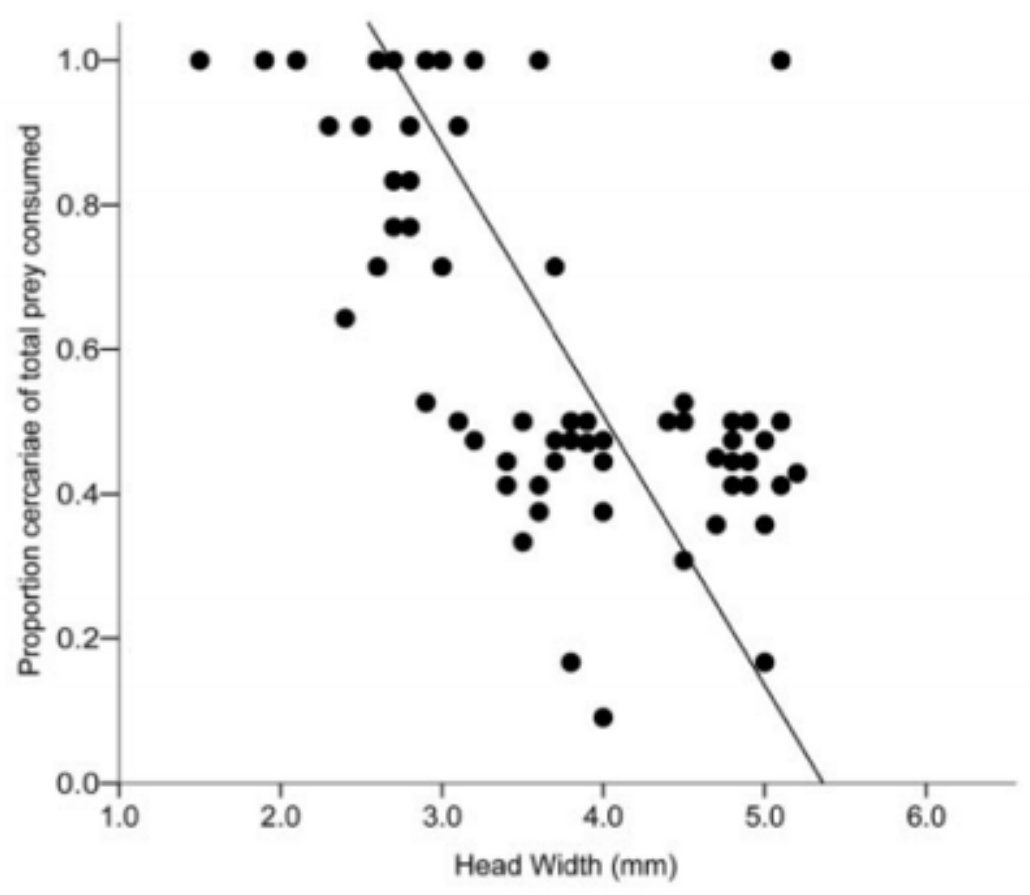

Figure 4.2. Proportion of Cotylurus sp and Posthodiplostomum sp. cercariae eaten by Leucorrhinia intacta dragonfly larvae across a range of predator head widths (1.5 - $5.2 \mathrm{~mm}$; $\mathrm{n}=$ 72). There was a significant negative relationship between larval dragonfly head width and proportion of cercariae consumed (Catania et al. 2016). Selecting larvae of 4-6 mm for my experiment thus encompassed the range of L. intacta consuming cercariae. 


\section{Lipid Analysis}

Whole odonates were freeze-dried and the moisture $\%$ of the tissue was calculated. They were individually ground into a fine powder in liquid nitrogen using a mortar and pestle, and the sample was then weighed on an analytical micro-balance (Mettler Toledo \#xp6, Mississauga, CA) with $1 \mu \mathrm{g}$ precision. The total lipid of each odonate were then extracted and the FAME were identified based on the previously described protocol (Chapter 2: Methods and Materials - Lipid Extraction).

\section{Statistical Analysis}

Prior to selecting the appropriate analyses, all of the data were tested for normality, homogeneity and independence of residuals. From these tests an Arcsin(sqrt) transformation was performed on percent content data (\% composition) of SFA, MUFA, PUFA as well as LNA, ALA, ARA, EPA and DHA since this transformation resulted in normally distributed data (McDonald 2008). No data transformation was applied to the absolute FA data ( $\mu \mathrm{g}$ of FA/mg DW).

I first calculated the growth of each larva by using the following equation: = ((final head width - start head width)/start head width). To assess the effect of diet on dragonfly larvae $\%$ growth, I used a t-test, pooling the data from the two trials, as trial was found to have no effect on dragonfly larvae growth $\left(\mathrm{t}_{37}=1.58, \mathrm{P}=0.1228\right)$. To test the hypothesis that diet had an effect on the SFA, MUFA and PUFA content of the dragonfly larvae, I used two separate linear mixedmodels with either MUFA or PUFA content as the dependent variable, diet as a fixed categorical factor, and trial as a random categorical factor. Trial was included in these models as it was found to have a significant effect on MUFA and PUFA content via two separate two-sample ttests. This difference may have been due to the slight increase in temperature between the two trails, as one was earlier in the summer, and the lab may have been a few degrees warmer in the first trial. Potentially, this resulted in a decrease in the PUFA of the growing algae, and a lower nutritional value for the Daphnia spp. Additionally, the snail hosts may have encountered more stress, as it was later in the summer for the second trial and this reduced their composition of PUFA/MUFA in the cercariae. Trial was found to have no significant effect on SFA content, therefore a two-sample t-test model was used to assess the effect of diet. All analyses were performed using SAS Enterprise Guide 61. 
To test the hypothesis that diet had an effect on the overall EFA content of dragonfly larvae, I used a multivariate analysis of variance (MANOVA), with diet as a fixed categorical effect and proportional values for ALA, LNA, EPA, ARA, and DHA as the dependent variables. The MANOVA analysis was performed using R Studio version 3.4.1.

\section{Results}

Diet and Growth

A full FA profile of the diet item $R$. ondatrae cercariae (proportional and absolute data) was conducted and discussed in Chapter 2 (Appendix Table A.1). When discussing significant differences hereafter, they were reported at the $\mathrm{P}<0.05$ level. There was no effect of diet on $\%$ growth of dragonfly larvae $(\mathrm{t}=-1.40, \mathrm{df}=37, \mathrm{P}=0.170)$, indicating that $L$. intacta achieved similar growth regardless of the prey upon which it fed. The mean $( \pm$ S.D.) \% growth of larvae fed $R$. ondatrae was $4.36 \pm 1.61$, and that of those fed Daphnia spp. was $3.44 \pm 2.44$ (Fig. 4.3).

\section{Diet and Fatty Acid Composition}

Dragonfly larvae fed either $R$. ondatrae or Daphnia spp. showed no difference in the percent content (\% composition) MUFA or PUFA (Fig. 4.4, Appendix Table A.2) However, a difference was found in the SFA (\%) content between $R$. ondatrae- and Daphnia spp--fed odonates (Fig. 4.4, Appendix Table A.2), with those feeding on $R$. ondatrae containing more SFA. Diet had no overall effect on the proportional EFA (\%) content (combined, ALA, LNA, EPA, ARA and DHA) of dragonfly larvae ((Fig. 4.5; Table 3.3). The follow-up univariate ANOVAs also revealed no effects of diet for proportional composition of ALA, LNA, EPA, ARA or DHA. There were also no differences in the absolute composition ( $\mu \mathrm{g}$ of FA/mg DW) of L. intacta larvae fed Daphnia spp. or R. ondatrae diets for SFA, MUFA and PUFA (Fig. 4.6, Appendix Table A.4). Diet had no overall effect on the absolute composition ( $\mu$ g of FA/mg DW) of EFA (Fig. 4.7; Table Appendix Table A.5), with follow-up univariate ANOVAs also revealing no effects on ALA, LNA, EPA, ARA or DHA. 


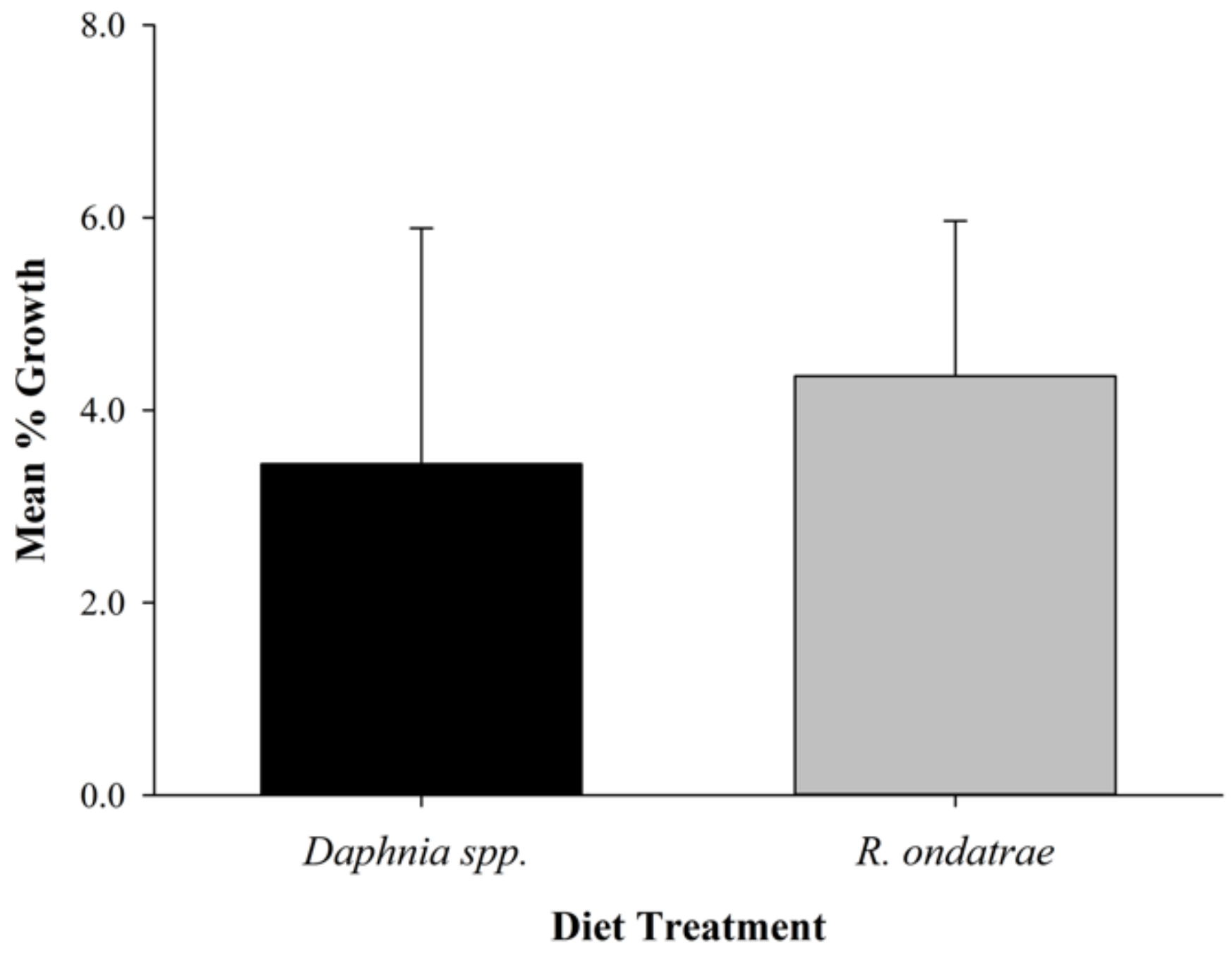

Figure 4.3. Mean ( \pm S.D.) \% growth (head width change) over 5 weeks of dragonfly (Leucorrhinia intacta) larvae fed one of two different diet treatments (Daphnia spp. or Ribeiroia ondatrae). No significant difference was found between the two treatments. 


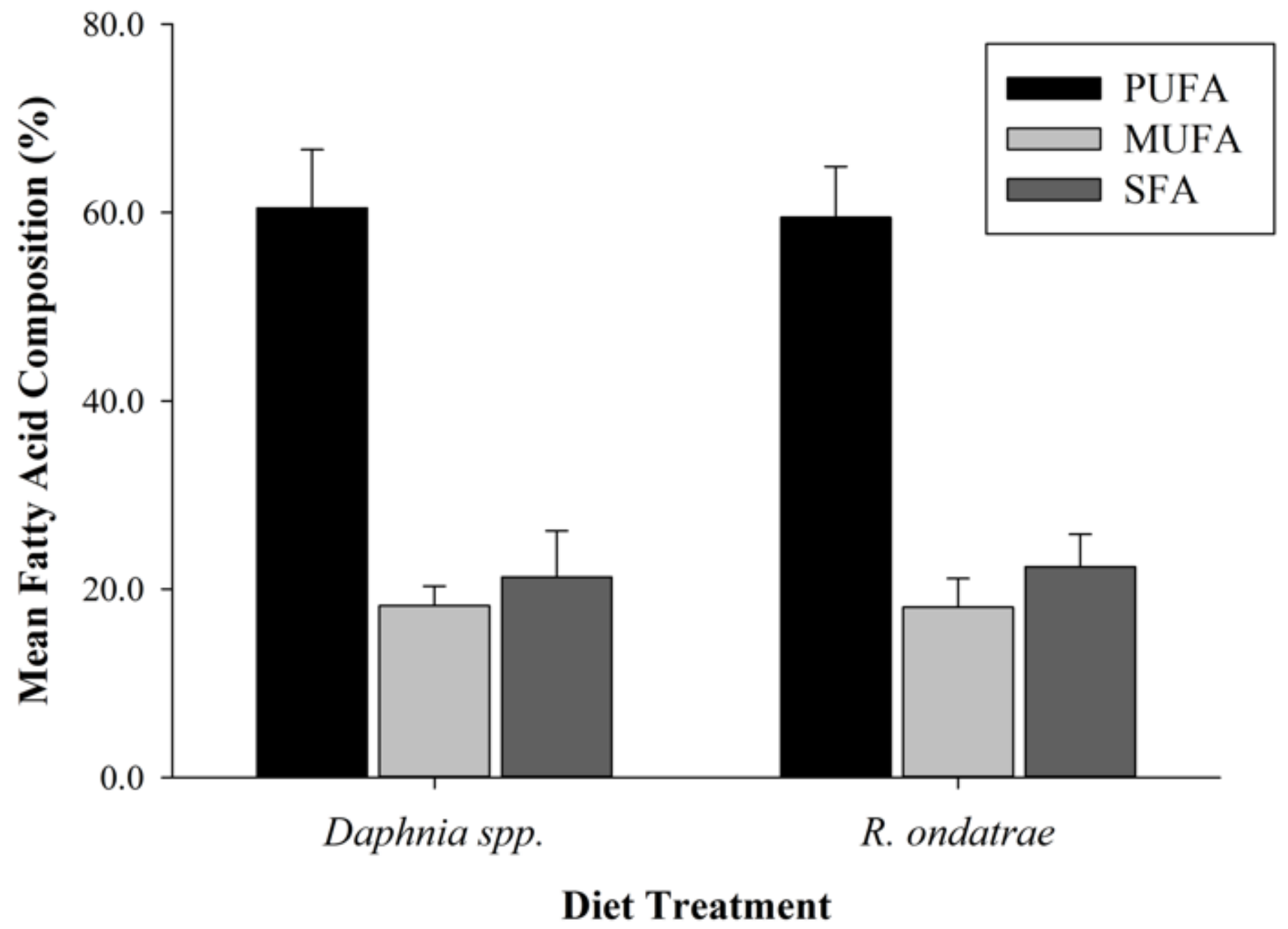

Figure 4.4. Mean ( \pm S.D.) proportional SFA, MUFA and PUFA composition of dragonfly (Leucorrhinia intacta) larvae fed one of two diet treatments (Daphnia spp. or Ribeiroia ondatrae). No significant difference was found between the treatments. 


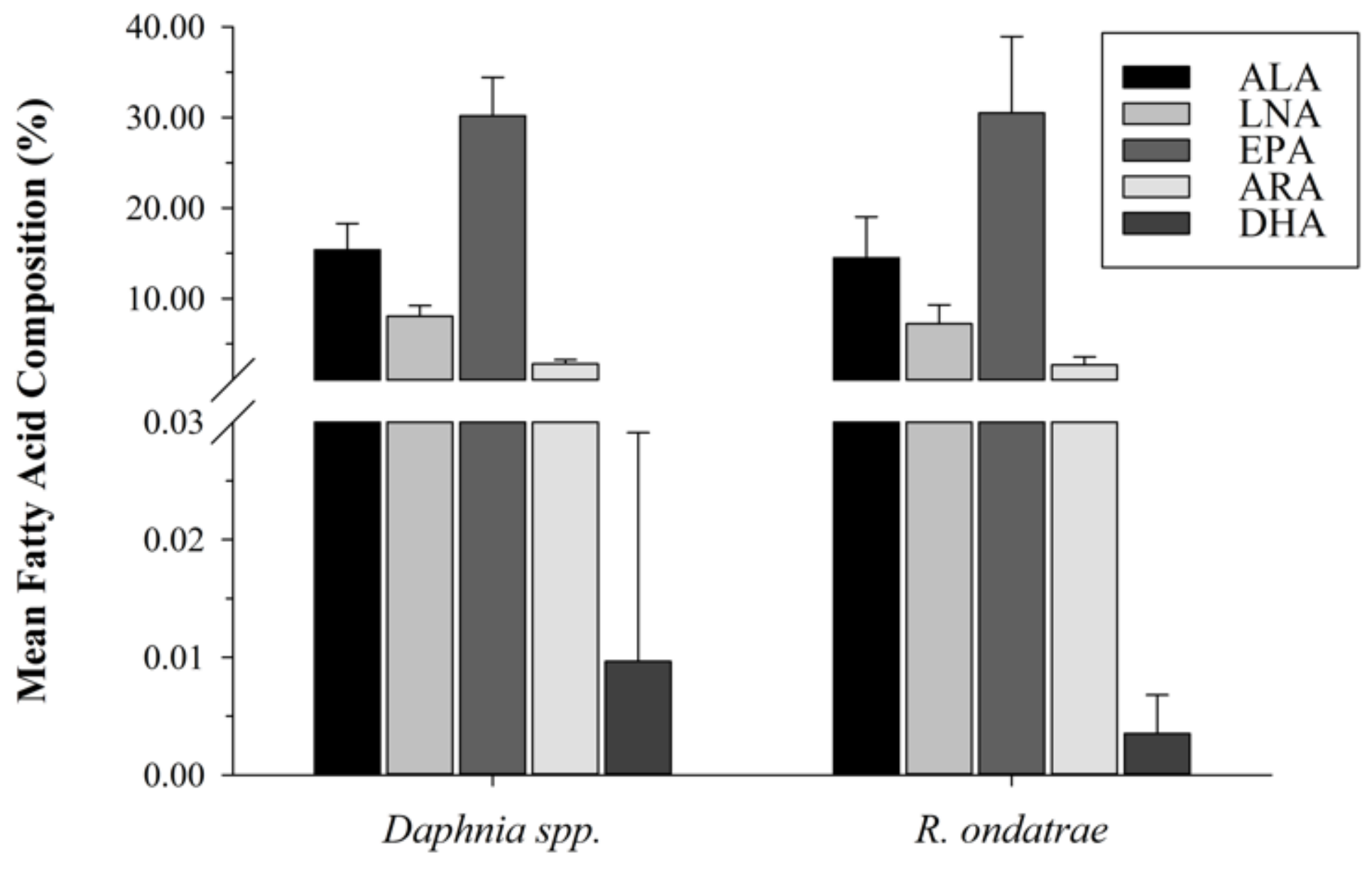

Diet Treatment

Figure 4.5. Mean ( \pm S.D.) proportional essential fatty acid (ALA, LNA, EPA, ARA, and DHA) composition of Leucorrhinia intacta fed two diet treatments. 


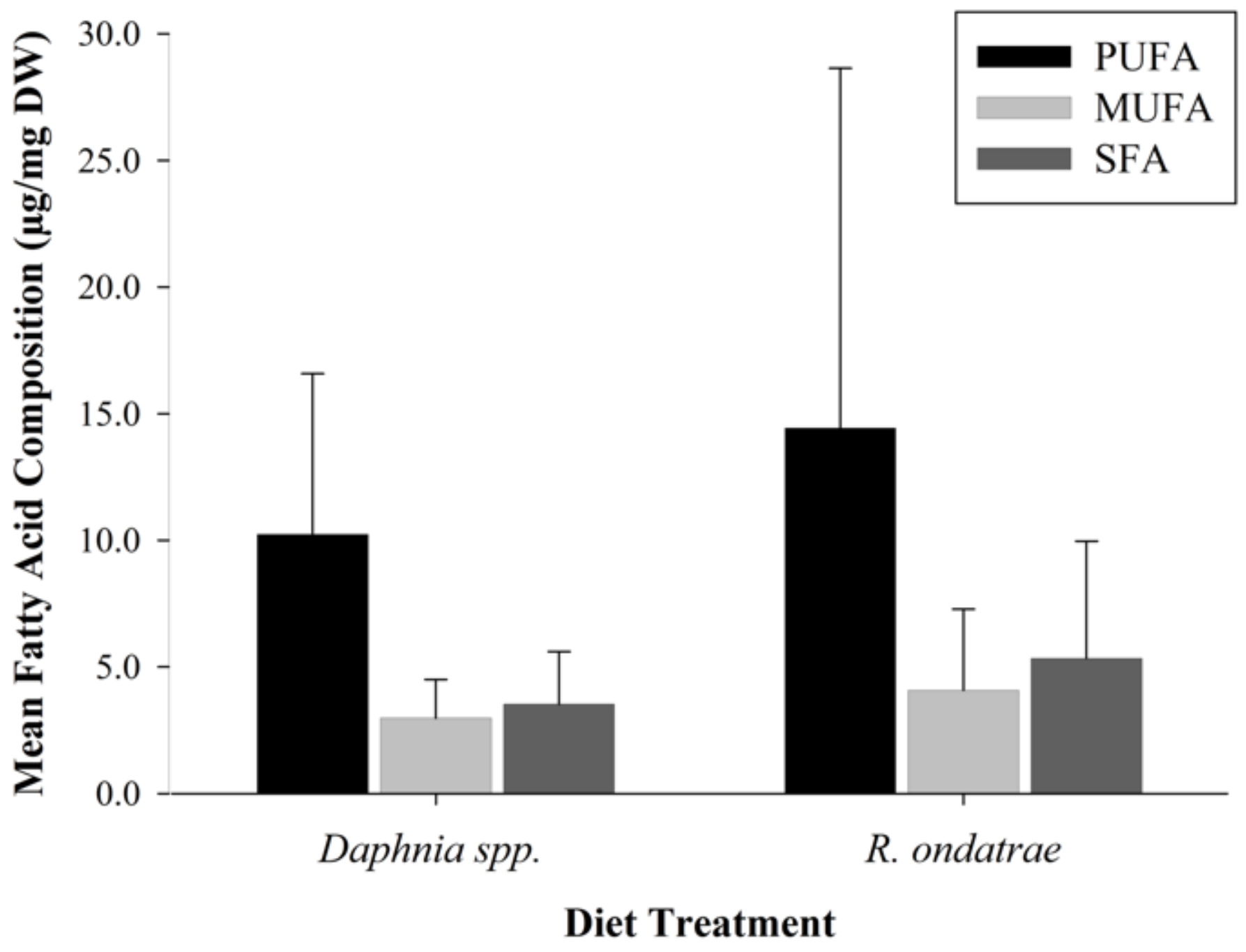

Figure 4.6. Mean ( \pm S.D.) absolute PUFA, MUFA and SFA composition ( $\mu \mathrm{g}$ of FA/mg DW) of dragonfly (Leucorrhinia intacta) larvae fed one of two diet treatments (Daphnia spp. or Ribeiroia ondatrae). 


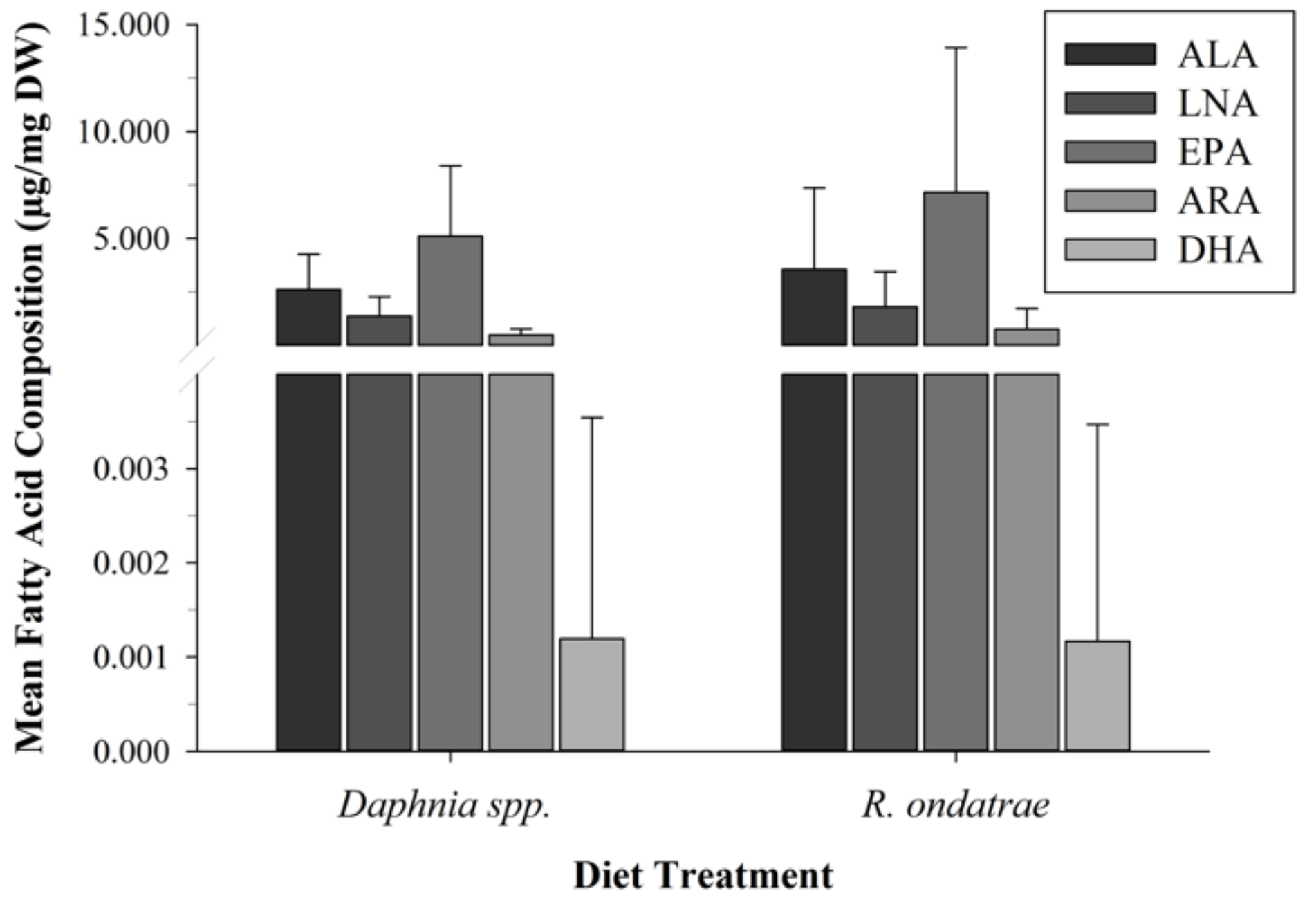

Figure 4.7. Mean ( \pm S.D.) absolute essential fatty acid (ALA, LNA, EPA, ARA, and DHA) composition ( $\mu \mathrm{g}$ of FA/mg DW) of dragonfly (Leucorrhinia intacta) larvae fed two diet treatments. 


\section{Discussion}

I demonstrate the equivalency of $R$. ondatrae cercariae and Daphnia spp. to serve as nutrientrich prey for a top freshwater predator, dragonfly larvae (Leucorrhinia intacta). Importantly, my results provide the first demonstration of the nutritional contribution of $R$. ondatrae cercariae to predator diets, including a quantitative comparison to common freshwater zooplankton. My results confirm previous findings that $R$. ondatrae cercariae are preyed on by small aquatic invertebrates; however, they also demonstrate that these cercariae are a nutrient-rich food source which can support the growth of an important aquatic predator. Leucorrhinia intacta larvae displayed similar \% growth, as well as FA compositions (proportional (\%) data as well as absolute ( $\mu \mathrm{g}$ of FA/mg DW) for MUFA, PUFA as well as ALA, LNA, EPA, ARA and DHA, whether they consumed either $R$. ondatrae or Daphnia spp. This suggests that $R$. ondatrae cercariae and Daphnia spp. serve as comparably nutritious prey items to aquatic invertebrates.

Recently, there has been a cry for the inclusion of parasites in food web theory as their addition alters key food web metrics, such as connectance and robustness (Marcogliese and Cone 1997; Lafferty et al. 2006; Lafferty et al. 2008; Byers 2009; reviewed by Sukhdeo 2012). Despite this, a previously unrecognized, but increasingly important role for free-living parasite stages, such as trematode cercariae, is their ability to serve as prey for aquatic organisms (Thieltges et al. 2013; Catania et al. 2016). Since this realization, investigations of their consumption by aquatic predators and subsequent implication on food web metrics, as well as the quantification of their immense biomass, have become more prevalent (Marcogliese 2003; Lafferty et al. 2006; Kuris et al. 2008; Lafferty et al. 2008; Johnson et al. 2010; Preston et al. 2013; Thieltges et al. 2013). However, studies have yet to explore how the substantial biomass of cercariae, and their deliberate and common consumption by aquatic predators, contributes to nutrient flow in food webs (Johnson et al. 2012). Previous studies have suggested similarities between cercariae and zooplankton as they are comparable in size, serve as prey for a variety of the same aquatic invertebrates, and typically reside in large dense populations (Morley 2012; Orlofske et al. 2012, 2015; Thieltges et al. 2013; Catania et al. 2016).

While it has been suggested that cercariae and zooplankton may be comparable in many ways, this had yet to be shown empirically (Morley 2012). These similarities may be due to the similarities in size, composition and diet of $R$. ondatrae cercariae and zooplankton such as 
Daphnia spp. For instance, many cercariae exhibit continuous active movement and typically reside in large populations, likening them to zooplankton (Morley 2012). Cercariae are also considered to be a free-living planktonic stage, making them analogous to meroplankton (planktonic larvae of benthic organisms) that typically fall in the size range of mesoplankton (0.2-2 mm) (Morley et al. 2012). Importantly, cercariae overlap in size with many zooplankton and zoobenthos fauna and lack an exoskeleton, which likely accentuates their suitability/palatability as prey (Ebert 2005; Orlofske et al. 2015). These observations are supported by previous studies which demonstrated that cercariae and zooplankton are preyed on by a similar range of benthic and planktonic aquatic animals including bryozoans, rotifers, cladocerans (e.g., Bythothrephes and Cercopagis), copepods, oligochaetes, Hydra sp., the aquatic larvae of damselflies and dragonflies, as well as predatory fish (Tokobaev et al. 1979; Stoddart 1982; Schotthoefer et al. 2007; Theiltges et al. 2008; Kaplan et al. 2009; Catania et al. 2016). Catania et al. (2016) specifically showed that predation on cercariae by L. intacta larvae is common and deliberate, as these were readily attacked and consumed even when provided with Daphnia spp. as an alternative food source.

Here, L. intacta larvae were not offered the option of either prey item, and their size range, as well as that of the cercariae, differed. Catania et al. (2016) used L. intacta larvae with head widths 1.5 - $5.3 \mathrm{~mm}$ vs. 4.2 - $6 \mathrm{~mm}$ here and fed them cercariae (Cotylurus sp and Posthodiplostomum sp.) $\sim 192 \mu \mathrm{m}$ long vs. $800 \mu \mathrm{m}$ for $R$. ondatrae in the current study. By selecting a smaller range of dragonfly larvae size, larger cercariae, and controlling for Daphnia spp. size $(0.75-1 \mathrm{~mm})$, I eliminated the potential for the consumption of either prey item to be influenced by predator handling preference (Catania et al. 2016). Additionally, Welsh et al. (2017) found that increased cercarial density resulted in higher levels of consumption, where the removal of cercariae by predators is directly linked to the infection levels downstream in secondary hosts (Liddell et al. 2017). These results further contribute to the suggestion that cercarial predation has effects on the disease risks of hosts, and that the species that compose the predator community will alter the survival of cercariae, and therefore the prevalence of infection (Welsh et al. 2017).

Previously (Chapter 2), I found that cercariae contain significant quantities of EFA, including EPA, DHA and ARA, as well as the shorter chain FA, LNA and ALA (Appendix Table A.1). The importance of identifying significant quantities of EFA in cercariae becomes significant 
when considering their ability to contribute to predator diets. Essential fatty acids are key nutrients used to assess food quality, and subsequently, potential for somatic growth and reproduction of organisms in freshwater ecosystems (Parrish et al. 1999; Kainz et al. 2004). These lipids have critical roles in the health and survival of consumers, as they cannot be biosynthesized with an efficiency that meets organismal needs (Arts et al. 2001). Essential fatty acids are thus produced in high quantities at the base of the aquatic food web mainly by phytoplankton (and, to a lesser extent, by aquatic fungi), and subsequently consumed and transferred to higher trophic levels (Gladyshev et al. 2008; Libkind et al. 2008). These LC-PUFA subsidies are therefore critical for maintaining optimal growth, survival, and reproductive rates for a wide variety of freshwater organisms, including Daphnia (Brett and Müller-Navarra 1997) and copepods. Specifically, the biomass of EPA and DHA in invertebrates is used as an indicator of their nutritional value for fish or other representatives at the top levels of food chains (Arts and Kohler 2009; Ahlgren et al. 2009; Gladyshev et al. 2016; Twining et al. 2016). As such, the availability of EPA and DHA in aquatic ecosystems has implications for the health and survival of primary and higher-level consumers as they play a vital role in cell membrane properties, serve as precursors for crucial animal hormones, and have a critical role in the development and function of neural and ocular tissue (Shul'man and Yuneva 1990; Innis 2007; Brenna et al. 2009; Farooqui 2009; Parrish 2009; Lands 2009; Simopoulos 2011).

While recent research on the predation of parasites has focused on the consequences for parasite transmission to the next host, as well as understanding prey selection (Orlofske et al. 2012; Thieltges et al. 2013; Catania et al. 2016), my results represent the first quantitative demonstration that cercariae function as important, nutrient-rich, prey items in both aquatic and, eventually, terrestrial ecosystems. I found similarities in the proportions and absolute composition of MUFA and PUFA, as well as EFA (including EPA, DHA, and ARA and shorter chain ALA and LNA), in dragonfly (Leucorrhinia intacta) larvae that consumed $R$. ondatrae cercariae or Daphnia spp. The similarities in the FA profiles of the larval odonate predator consuming both diets can be explained by examining the FA profile of $R$. ondatrae cercariae (previously determined in Chapter 2) to published zooplankton FA profiles. Critically, similarities in FA profiles indicate the ability for cercariae to serve a similar function to zooplankton in their role of transferring EFA and biologically active lipid precursors from the base of aquatic food webs to higher trophic levels, thereby supporting ecosystem health, 
productivity, and resilience. Additionally, as L. intacta emerge onto the terrestrial landscape as adults, they bring with them aquatically-derived lipid subsidies from prey consumed as larvae, which must now include $R$. ondatrae cercariae as shown here. As such, through emergent insects or other channels, $R$. ondatrae cercariae contribute EFA to not only aquatic, but also terrestrial, organisms, thereby supporting growth and development in both ecosystems.

Zooplankton serve as an important pathway for the transfer of EFA from phytoplankton primary producers to higher trophic level consumers (Müller-Navarra 2006). Zooplankton FA composition is highly reflective of their diet, and as such, the similarities in the FA profiles of $L$. intacta that fed on zooplankton or cercariae may be reflective of their algal diets (Arts et al. 2009). Both prey items consumed Chlamydomonas sp. algae, either through direct consumption by Daphnia spp., or indirectly for the cercariae as their first intermediate hosts (aquatic gastropods) consumed algae and transferred nutrients to their tissues, which were then consumed by rediae that contained developing cercariae. Therefore, the similarities in the FA profiles of the larval dragonfly predators fed either diet indicates that both prey items were accumulating transferred algal FA. Here, $R$. ondatrae cercariae likely would have obtained EFA through the consumption of host tissue, a planorbid snail, whose diet consists primarily of algae (Lombardo et al. 2002). Chlamydomonas sp. algae contain high quantities of ALA and LNA and snails are capable of desaturating and elongating shorter chain FA into LC-PUFA (Van der Horst 1973). The composition of snail hosts has been previously found to reflect that of their diet, as seen in an experiment where $H$. trivolvis which consumed lettuce leaves were found to contain high amounts of ALA, the most abundant FA in that food item (Oudejans and Van der Horst 1974; Fried et al. 1992b).

Consequently, similarities in L. intacta larvae composition reflect the ability for cercariae to trophically transfer EFA from primary producers to higher trophic level consumers, but may also reflect parallels in the preferential accumulation of FA in both cercariae and zooplankton. The FA composition of zooplankton is influenced by that of their diet, as well as the ability of some species to selectively accumulate and metabolically alter dietary EFA, leading to variable, although generally conservative, FA profiles (Weers et al. 1997; Von Elert 2002; Graeve et al. 2005; Brett et al 2006a,b; Müller-Navarra 2006; Veloza et al. 2006; Smyntek et al. 2008; Ravet et al. 2010). As such, zooplankton contain high quantities of EFA due to their algal diets, which is a key driver of ecosystem productivity and organism growth (Ahlgren et al. 1990; Müller- 
Navarra 1995; Brett and Müller-Navarra 1997; Müller-Navarra et al. 2000; Sikora et al. 2016). Specifically, EPA shows a particularly strong correlation between diet and Daphnia FA composition (Brett et al. 2006b; Müller-Navarra 2006; Arts et al. 2009). Therefore, this may indicate that cercariae preferentially accumulate EPA as the compositions of the predators given different prey were similar and reflect that of their algal diet. Interestingly, dragonfly larvae exhibited differences in proportional SFA composition, with $R$. ondatrae fed larvae containing higher proportions (Fig. 4.4). This observation may be explained by innate differences in the SFA composition of $R$. ondatrae cercariae and Daphnia spp. (Fig. 4.3a). Daphnia may have a tendency to accumulate less SFA, but more MUFA and ARA, than what is found in their diets (Arts et al. 2009). As such, cercariae may accumulate SFA at concentrations that are more reflective of their diet composition, which may have resulted in higher SFA compositions in their larval odonate predators.

In discovering that cercariae contribute EFA to higher trophic level organisms, I highlight a previously unknown role of cercariae in food web dynamics and ecosystem productivity. Cercariae are similar in biomass to free-living species of comparable size and trophic level (Kuris et al. 2008; Hechinger et al. 2011; Thieltges et al. 2013). Their biomass in some freshwater pond ecosystems has been likened to that of an Asian elephant, with annual biomass significantly exceeding that of principal top ecosystem predators and comparable to major groups of free-living species larger in individual body size (Kuris et al. 2008; Soldánová et al. 2016). Consequently, they may represent significant sources of energy and nutrients for consumers. For instance, it was estimated that cercariae support 2-3\% of the total energetic demands of the fish community in a California estuary (Kaplan et al. 2009).

The consideration of temporal variation in the timing of cercariae and Daphnia spp. peak abundances is needed when comparing their ability to serve as comparable prey items. Cercarial emergence is linked to the warm water temperatures and, as such, they can only be detected during the warmer months of the growing season (Morley et al. 2010). Specifically, cercariae continue to emerge from snails once the temperature exceeds $4-10^{\circ} \mathrm{C}$ in temperate latitudes, rising to $15-16^{\circ} \mathrm{C}$ in tropical regions (e.g., Pfluger 1980; Lyholt and Buchmann 1996). Cercarial production can remain more or less constant once these temperaturethresholds are exceeded. In contrast, zooplankton populations typically show a spring bloom in spring months prior to the cercarial threshold temperature, before declining (Burns 1992). As such, cercariae may serve as 
an especially important food source at particular time points throughout the late spring, summer and eaerly autumn months. This potential temporal disconnect needs further investigation but may allow cercariae to buffer potential shoratges in energy and nutrients for their predators.

In lakes, the majority of freshwater fish (up to $93 \%$ of species) rely on benthic resources in the littoral zone for nutrients (Vadeboncoeur et al. 2011; Lau et al. 2012), and may therefore be receiving significant amounts of EFA from the consumption of cercariae given their significant biomass. In fish, EPA and DHA are used as criteria to indicate high nutritional value, which has important consequences for the health of humans, as LC-PUFA are not synthesized in sufficient quantities in humans to maintain optimal growth (Arts et al. 2009). As both EPA and DHA are produced in high concentrations in algae, it is vital to understand the dynamics of LC-PUFA at the base of aquatic food webs as they are key drivers of aquatic ecosystem productivity (MüllerNavarra 1995; Müller-Navarra et al. 2000). Considering that EPA and DHA are limiting resources in aquatic ecosystems, the discovery that cercariae may be supplying considerable quantities of both to aquatic predators (Appendix Table A.1) has significant implications for their growth and the productivity of aquatic ecosystems, as well as that of terrestrial organisms. Further, it highlights the ability for cercariae to serve as a bridge for the transfer of EPA and DHA across the plant-animal interface, which is vital in ensuring that these essential nutrients are accessible for consumption by aquatic predators.

Aquatic and terrestrial landscapes are inherently linked through the transfer of nutritional subsidies between the two proximal habitats (Martin-Creuzburg et al. 2017). As aquatic ecosystems provide EFA for not only aquatic organisms, but also terrestrial, identifying new pathways for their transfer from the base of the aquatic food web is vital. LC-PUFA are physiologically vital biomolecules that can critically affect the productivity and survival of all vertebrates, and it is therefore essential to identify and further understand the major trophic links contributing to these nutrient fluxes in both aquatic and terrestrial ecosystems (Polis and Hurd 1996; Nakano and Murakami 2001; Gratton and Zanden 2009). Given that terrestrial insects contain no or negligible amounts of EPA and DHA (Buckner and Hagen 2003; Wang et al. 2006), emergent insects are a vital source of EFA for terrestrial landscapes. My results highlight $R$. ondatrae cercariae as a source of LC-PUFA for emergent insects which may be contributing to the health and survival of terrestrial organisms, as well as that of aquatic consumers. 
The ability for cercariae to serve as nutrient-rich prey solidifies the importance of understanding their roles in food webs. The understanding that free-living infectious stages such as cercariae can serve as a potentially equivalent food source to abundant zooplankton (Daphnia spp. here) further advances the case for their inclusion given that recent studies have reported that parasites alter food web structure through an increase in diversity and complexity, as well as uniquely alter the structure and function/dynamics of the system (Lafferty et al. 2008; Dunne et al. 2013). With their ability to support organism growth and contribute to essential lipid transfer, the inclusion of cercariae, in particular, is crucial to gain a complete understanding of ecosystem function. 


\section{CHAPTER 5: SUMMARY AND FUTURE DIRECTIONS}

The concept of food webs is central to the field of ecology and is a key principle underlying our understanding of the factors that control ecosystem stability, diversity and complexity (McCann 2000; de Ruiter et al. 2005; Montoya et al. 2006; Pascual and Dunne 2006; Bascompte 2009; Thompson et al. 2012). Over the past two decades, it has become increasingly apparent that parasitism, one of the most common consumer strategies, uniquely alters food web properties through its ability to increase both connectance and diversity (Marcogliese and Cone 1997). Additionally, parasites distinctively affect food web structure and function/dynamics due to their unique roles, and which include: a) serving as resources for predators of their hosts, b) possessing broad feeding niches due to their complex life cycles, and, c) demonstrating the inverse of the typical relationship between predator and prey body size (Lafferty et al. 2008; Sukhdeo et al. 2012; Dunne et al. 2013). Typical food web models focus on the relationship of prey to predator body size, where consumers eat prey smaller than themselves (Cohen et al. 1990; Williams and Martinez 2000; Cattin et al. 2004; Dunne et al. 2013). Parasites fit poorly into current food web models since they rely on energy from larger (host) organisms (Dunne et al. 2013). Recently, the incredible biomass of parasites within ecosystems has begun to be quantified (Kuris et al. 2008; Hechinger et al. 2011; Dunne et al. 2013). For instance, snails parasitized by trematodes emit millions of free-living cercariae into the aquatic environment on a daily basis, with biomasses in small ponds estimated to be upwards of 4.65 tons annually, equivalent in mass to an Asian elephant (Soldánová et al. 2016)!

From a food web perspective, free-living parasite stages, such as trematode cercariae, may play an important role when considering their potential to serve as prey items for higher trophic level predators. Cercariae comprise an immense biomass in aquatic ecosystems, and contribute substantially to zooplankton and detrivore communities as they are frequently preyed upon by a range of invertebrates and vertebrates (Tokobaev et al. 1979; Soddart 1982; Schotthoefer et al. 2007; Thieltges et al. 2008; Kaplan et al. 2009; Catania et al. 2016). Despite this, their roles in

food webs are currently understudied, particularly their ability to serve as nutritionally-valuable prey items (Johnson et al. 2010).

Therefore, my thesis work was designed to describe and quantify the potential nutritional contribution of trematode cercariae to freshwater ecosystems, and also to consider the wider 
implications of aquatic resource production in parasites as a key subsidy for adjacent terrestrial ecosystems. Specifically, I was interested in studying lipids that may serve as energy reserves in cercariae from a range of trematode species, as well as analyzing the fatty acid (FA) profile of Ribeiroia ondatrae cercariae. I also examined the impact of trematode infection on the FA composition of tissues from snails (Helisoma trivolvis) that harbored $R$. ondatrae infections compared to uninfected controls. Additionally, I wanted to explore the ability for $R$. ondatrae cercariae to serve as nutrient-rich prey relative to zooplankton for the larval dragonfly Leucorrhinia intacta, thereby supporting the growth and productivity of a top aquatic ecosystem predator that eventually, in the adult stage, emerges onto terrestrial landscapes.

In Chapter 2, my first objective was to develop a semi-quantitative histochemical technique to investigate individual differences in neutral lipid energy reserves among cercariae from different trematode taxa, as well as produce a quantitative FA profile for $R$. ondatrae cercariae. I hypothesized that the five cercariae taxa/types (R. ondatrae, Echinostoma sp., Haemoloechus sp., armatae-type and strigeid-type cercariae) would differ in their lipid distribution and quantity as these cercariae vary considerably in their body structures and organs, thus different tissues may have different FA compositions. As cercariae from different taxa may acquire nutrients via different mechanisms (i.e. rediae vs sporocysts within snail hosts - see Chapter 1), and could have varied biosynthetic capabilities (i.e. some species may be more adapted to synthesize more and/or different lipids), I hypothesized that this could also contribute to variation in neutral lipid stores within cercariae.

The five trematode cercariae taxa/types differed greatly in the localization of their lipid droplets as expected; however, lipids were frequently localized to organs that support excretion and secretion, suggesting that lipids play a role in the penetration process in order to promote successful infection of their next host (Zd'árská 1979). Cercariae of Haematoloechus sp., one of the smallest investigated here, contained the highest quantity of neutral lipids, while larger cercariae, such as Echinostoma sp. and R. ondatrae contained intermediate neutral lipid levels. I suggest that individual differences in lipid localization and composition may be driven by a variety of factors which together produce distinct patterns among cercariae taxa. I further suggest that one such driver is the presence or absence of specialized organs (i.e. excretory ducts) that secrete and utilize lipids for functions including aiding in host penetration (Haas et al. 1997). Moreover, lipids may contribute to buoyancy whereby the position and distribution of lipid 
droplets within cercariae may correspond to their swimming patterns and relative position in the water column (reviewed in copepods by Sargent and Henderson, 1986) and help to facilitate transmission in a host-specific manner (Combes et al. 1994).

While neutral lipid stores differed distinctly among cercariae type/taxa, and I observed some taxon-specific patterns, future work should continue to investigate energy reserve lipids in relation to unique traits among cercariae taxa. To tease apart these factors, studies should aim to investigate numerous cercariae from multiple families that display distinctive swimming intensities, particularly tail movement and position in the water column (both in relation to the substrate or surface, as well as the maintenance of a vertical or horizontal position), to determine whether these behaviors relate to parasite lipid composition. Such investigations of overarching patterns in lipid composition among cercariae from different taxa will contribute to our understanding of their function in these larval trematodes, an area of study that has yet to be entirely explored. Additionally, further research expanding on the role of individual fatty acids in the penetration success of parasites would be particularly fascinating, especially considering their potential to possess an active FA metabolism. Given that cercariae receive nutrients from their snail hosts, whose composition reflects that of their diet, environmental and dietary influences on snails may also have important consequences for parasite longevity, penetration and behaviour that should be investigated.

In the second part of Chapter 2, I investigated the FA profile of an aggregate sample of $R$. ondatrae cercariae ( $\sim 30000)$. I had hypothesized that cercariae likely contain significant quantities of essential fatty acids (EFA), and may therefore be contributing substantial quantities of these key nutrients to aquatic ecosystems. My rationale for this hypothesis was based on the fact that cercariae rely solely on nutrients from their snail hosts during their development, and that snails contains significant quantities of EFA which are obtained from their diet (Lombardo et al. 2002). Snails are algivorous, therefore they consume algae, some species of which contain high quantities of EFA (Hill et al. 2009). Snail hosts may thus be passing EFA to the cercariae developing within them, and cercariae may be serving as a novel source of these essential nutrients if they are consumed upon emergence. I found that $R$. ondatrae cercariae contained significant quantities of EFA, thereby identifying a previously undescribed source for aquatic ecosystems. I suggest that cercariae may be absorbing host tissue FA, and may additionally possess the potential to synthesize FA de novo. Cercariae in other taxa, such as the species 
Schistosoma mansoni, contain lipases to convert host lipids into eicosanoids, as well as an active arachidonate metabolism (Mandlowitz et al. 1960; Fusco et al. 1985), illustrating that cercariae utilize host lipids. Additionally, the Phylum Platyhelminthes is closely related to the Phylum Gnathifera, which contain the necessary genes to synthesize long-chain, biologically active FA (Kabeya et al. 2018). The potential consequences of de novo synthesis are clear when we consider my calculations that $R$. ondatrae cercariae alone may contribute $\sim 24 \mathrm{mg} / \mathrm{m}^{2} / \mathrm{yr}^{-1}$ of EPA and $\sim 0.4 \mathrm{mg} / \mathrm{m}^{2} / \mathrm{yr}^{-1}$ DHA in a typical pond. These estimates were done on data from a freshwater pond, however the contribution of EFA from cercariae in marine systems (specifically salt marshes) may be even more important due to the extended production season, and extremely high prevalence of infected snails (Thieltges et al. 2008). Additionally, if snails can also synthesize FA de novo, then cercariae may be in the unique position to trophically-upgrade FA. Notably, various members of the Phylum Mollusca were found to possess the necessary genetic machinery (Kabeya et al. 2018). In observing and quantifying their potential to contribute to aquatic ecosystem energetics and nutrient transfer, I demonstrate the indisputable need for the addition of parasites to food webs, as well as provide a quantifiable metric to do so. As we become more aware of the potential for cercariae to serve as nutritious prey items, future work continuing to resolve their position within food webs and ability to not only act as predators, will help to completely understand and quantify ecosystem nutrient flow.

Future research should aim to further investigate the distribution, and composition of lipids (i.e. what molecular species of TAG, DAG) and to continue to quantify energy reserve lipids in a variety of cercariae species, as well as among the same species collected from different snails and/or different ponds. Additionally, addressing the potential far-reaching consequences associated with the effects of environmentally driven changes (i.e. climate warming) on the lipid composition of cercariae would shed light on the future potential of cercariae as a food source. Cercariae acquire their nutrients from snail hosts and their composition may reflect that of their snail host (Fried et al. 1992b). Increased temperatures decrease the lipid-related nutritional composition of algae through a process known as homeoviscous adaption, whereby cell membranes reduce the proportion of biologically important PUFA and increase the proportion of SFA present to maintain ideal membrane fluidity (Sinensky 1974). This alteration in the lipid composition of algae could lead to less nutritious snail tissue, and subsequently therefore a potential reduction in parasite nutritional value as related to FA. Increased temperatures have 
been found to significantly decrease the bioactive fatty acid stores (i.e. LC-PUFA and n-3 FA see Chapter 1) in snails (Deschasseaux et al. 2011). If cercariae are supplying the aquatic system with essential fatty acids, a decrease in their LC-PUFA content would affect nutrient flow within aquatic ecosystems. Paradoxically, increased temperatures also facilitate an increase in cercarial emergence, and may offset the potential reduction in LC-PUFA that would decrease the nutrientrich status of each individual cercariae (Johnson et al. 2002). Future work examining the effects of temperature on cercariae in relation to current and future temperature predictions, coupled with counts of cercarial emergence, may help to uncover their potential effects on food webs as well as parasite transmission. Additionally, further investigation into the effects of temperature on the metabolism of parasites, and the potential for increased temperature to alter their behaviour or partitioning of resources, would contribute the creation of a metabolic theory of ecology (MTE) framework for parasites in hosts (Hechinger et al. 2011; Hechinger 2013).

In Chapter 3, my objective was to examine the effect of $R$. ondatrae trematode infection on the lipid composition of its snail host, $H$. trivolvis. Trematode infections are localized to the gonadal regions of their snail hosts, and therefore reside in the digestive-gonad gland (DGG) complex (Crews et al. 1989). To elucidate the effects of $R$. ondatrae infection, a factorial design was used to compare the FA composition of $R$. ondatrae-infected DGG complexes and head/foot tissue compared to both tissue types in uninfected controls. I hypothesized that both tissue types from infected snails would contain higher quantities of neutral lipids and higher proportions of EFA compared to tissues from uninfected snails. Helisoma trivolvis tissue from infected individuals was found to contain significantly more lipids (mg of lipids/mg of tissue), as well as higher proportions of myristic, oleic, nervonic, adrenic, DHA and saturated fatty acids than the same tissue from uninfected controls. The increased lipid levels of infected snails, as well as presence of significantly more DHA in parasitized tissue (the DGG complex), suggests that trematodes may be producing and storing lipids through FA chain elongation and desaturation of host lipids (Schariter et al. 2002).

Future work should determine if de novo synthesis of FA, specifically LC-PUFA by cercariae (or the rediae and sporocysts in which they develop) is widespread, such as by investigating the presence of a $121 \omega \mathrm{x}$ desaturase sequence within the Phylum Platyhelminthes (Kabeya et al. 2018). Investigating how other factors (i.e. diet, temperature) affect DGG composition would help to further describe the use of host lipids by developing parasites. For instance, infected and 
uninfected snails maintained on egg yolk diets exhibited higher neutral lipid fractions (increased TAG) in their DGG complex compared to snails maintained on lettuce diets (Thompson et al. 1991). Additionally, lipid-rich diets shortened the period of patency for cercariae development, meaning that mature infective cercariae were ready to emerge from snails sooner (Thompson et al. 1991). However, another study that attempted to repeat these trials did not find a shortened time to patency (Fried et al. 2001). It would therefore be interesting to investigate the FA composition of snails maintained on lipid-rich and poor diets, as well as the effects of host diet and environmental conditions on the neutral lipid depots and FA profiles of emergent cercariae.

I demonstrated that $R$. ondatrae infection has distinct effects on the lipid composition of its gastropod host, in keeping with previous studies utilizing other host-parasite systems (Fried et al. 1989, 1993a,b, 1998b, 2001; Sherma and Fried 1990). Although not observed in my study, some trematode species have been shown to reduce snail lipids, inducing a greater cost to the host, and whereby subsequent exposure to additional stressors (e.g., temperature, estivation) may then pose an even greater challenge to infected snails. As climate warming is expected to increase temperatures, but decrease snail lipid stores (Deschasseaux et al. 2011), parasites that further reduce snail lipids may induce stress within infected snails, possibly leading to population decreases. A population decrease of infected snails, would be beneficial in the reduction of cercariae transmission to the second intermediate host. However, parasites that increase snail lipids may allow the snail to survive longer, ultimately producing more cercariae whereby potentially increasing transmission rates (Sousa, 1983). Nevertheless, increased cercariae production may also contribute substantial lipids to ecosystems throughout a snail's life time.

Additionally, parasitized snails may represent prey of higher nutritive value for their predators, and could contribute nutrients to food webs through predation upon these hosts (Sánchez et al. 2009). Increased lipids throughout the bodies of infected snails, coupled with the potential for cercariae to both ingest FA from their snail hosts and produce de novo synthesized FA of their own (Kabeya et al. 2018), highlight the possibility for this parasite-host relationship to produce substantial quantities of lipids in aquatic ecosystems. The exact reason why trematode-infected snails exhibit differences in lipid stores is unknown, therefore further work investigating the role of lipids in the parasite-host relationship is needed. The effects of trematode infection on snail lipids has important ecosystem-level implications as lipid stores could be drastically different among parasitized and un-parasitized snails, affecting their nutrient 
status as prey. This is particularly relevant when we consider the variation in parasite prevalence between habitats (Lefèvre et al. 2009), as heavily parasitized or un-parasitized ecosystems would experience either increases or decreases in the FA composition of aquatic snails.

In Chapter 4, I investigated the growth, total lipids, and FA composition of L. intacta larvae fed equivalent masses of either trematode cercariae ( $R$. ondatrae) or freshwater zooplankton (Daphnia spp.). This was done to assess the extent to which cercariae served as a nutrient-rich food source by comparing the growth of L. intacta fed the two different diets. In addition, I also investigated the potential for cercariae to transfer lipid subsidies from the base of aquatic food webs to higher trophic levels in both aquatic and, by extension, terrestrial habitats. Due to the similarities in the trophic level, size, and potential lipid composition of Daphnia spp. and $R$. ondatrae, I hypothesized that dragonfly larvae fed $R$. ondatrae would show similar growth and FA profiles to those fed the Daphnia spp. My results confirm previous findings that $R$. ondatrae cercariae are preyed on by small aquatic invertebrates (Orlofske et al. 2015; Catania et al. 2016). However, they also demonstrate that these cercariae are a nutrient-rich food source which can support the growth of an important aquatic predator since dragonfly larvae grew equally well on diets of either R. ondatrae or Daphnia spp, as well as contained similar EFA profiles.

In documenting this, I uncover a novel and substantial pathway through which LC-PUFA (see Chapter 1) are transferred to higher trophic level organisms in aquatic ecosystems. Terrestrial ecosystems also benefit because emergent insects are a critical vector through which LC-PUFA are transferred from the water to the land (Martin-Creuzburg et al. 2017). For instance, the dragonfly Leucorrhinia intacta studied here transfers EFA acquired during its aquatic larval stage to terrestrial ecosystems after metamorphosis to the aerial adult life stage (Gladyshev et al. 2009). The transfer of aquatic nutrient subsidies is vital to the survival of riparian predators such as spiders, lizards, birds and bats (Kato et al. 2004; Baxter et al. 2005; Burdon and Harding 2008). Riparian animals demonstrate increased fitness when they consume aquatically derived LC-PUFA. For example, wetland spiders with high tissue levels of aquatically derived LC-PUFA have elevated immune function compared to upland spiders (Fritz et al. 2017). Stable isotope analyses have also revealed that spiders in riparian zones of streams obtain the majority of their carbon and nitrogen sources from emerging insects (Collier et al. 2002; Baxter et al. 2005; Sanzone et al. 2003; Kautza and Sullivan 2016). Additionally, birds have FA profiles that are reflective of the FA composition of their diet (Scaife et al. 1994), and had reduced condition and 
reduced reproductive success in habitats where they consumed a high portion of terrestrial food (Hebert et al. 2002, 2008). Tree swallow chicks fed a direct LC-PUFA-rich diet were also found to grow faster and achieve better condition than chicks feeding on a LC-PUFA deficient diet (Twinning et al. 2016). From a nutritional perspective, aquatic insects therefore represent a superior food source relative to terrestrial insects as they provide consumers with essential lipid subsidies (Martin-Creuzburg et al. 2017). As I demonstrate, cercariae can contribute significant quantities of EPA and DHA to the diets of emergent insects while they are in their aquatic larval stage. The global export of EPA and DHA through insect emergence is estimated to be $240 \times 10^{6}$ $\mathrm{kg} \mathrm{yr}^{-1}$, and combined with other forms of export (i.e. direct predation on aquatic organisms), the

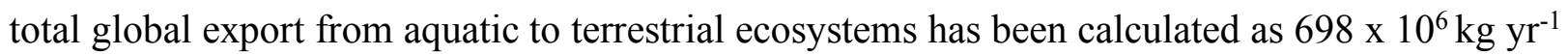
(Gladyshev et al. 2013).

In investigating the trophic transfer of essential lipids from cercariae to their predators, I suggest a potential food web schematic that prompts us to consider the free-living stages of parasites such as trematodes in the context of the flux of nutrients within aquatic and between aquatic and terrestrial ecosystems (Fig. 1.1, See Chapter 1). The most common strategy for the inclusion of parasites into pre-existing food web models involves tracing a given parasite through its intermediate and final hosts by considering its life cycle and transmission pathways. A much more realistic and powerful approach identifies parasite connections within trophic interactions in food webs (Price 1980; Marcogliese and Cone 1997; Marcogliese 2002), as life cycles by themselves do not necessarily provide the full picture. This is because life cycles frequently only consider parasite stages that are within hosts, leaving out the contribution of freeliving stages. Relying on life cycles also overlooks the potential for animals to interact with parasites or infected hosts through mechanisms that do not contribute to transmission (i.e. the consumption of infected hosts, consumption of free-living stages).

Future work should continue to investigate the roles of parasites in food webs as much information is still lacking. Critically, parasites still occupy a strictly consumer role, and have yet to also be more fully considered as a food source per se, and therefore a broader consideration of the role of parasites in aquatic systems is needed. Studies investigating the effects of trematode infection on the growth and lipid profiles of other predators that consume cercariae (e.g., oligochaetes, damselfly larvae, additional dragonfly larvae, and planktivorous fish) would further resolve which organisms may rely or consume cercariae for nutrients. Understanding the 
mechanisms through which EFA are transferred from snail hosts to parasites, and then from parasites to their predators, is an important step to begin quantifying their broad contributions to aquatic and terrestrial ecosystems.

The realization that cercariae contribute immense biomass and serve as prey in aquatic systems has fostered a growing recognition that parasites play an important role in food web energetics and nutrient flow. The results of my thesis began the exploration of quantifiable measurements to assess the impact of parasitism on some key ecological processes (i.e. energy and nutrient flow in food chains via predation on cercariae and infected hosts), and provide a foundation for further work in this area. To date, studies on parasitism in food webs have commonly been rooted in a natural history approach, focusing more on observational methods as opposed to the quantitative analysis of ecosystem processes. We are aware that parasitism affects ecological properties, and that it alters trophic links and energy transfer beyond just the hosts immediately involved in transmission; however, the broader implications of these ecosystemlevel processes have been rarely explored. The quantitative data presented here are essential for the logical next step of building food web models in a manner so as to understand the contribution of parasites to the flow of energy and nutrients within ecosystems wherein they support the stability and productivity of both aquatic and terrestrial habitats.

Essential fatty acids are integral to key physiological and biochemical processes that are vital for optimal ecosystem function. These EFA are highly conserved in aquatic ecosystems, as they are the primary source of EFA in which terrestrial organisms rely upon for their survival. In demonstrating that free-living infectious stages of trematode parasites (i.e. cercariae) are a route for the trophic transfer of essential lipids, I uncover a novel and previously undescribed mechanism which may be supporting the health and productivity of not only aquatic, but also terrestrial ecosystems. Future climate warming threatens the LC-PUFA composition (i.e. through the process of homeoviscous adaptation) of traditional sources (e.g., algae, fish) of EFA. Essential fatty acids have intrinsic importance to the health and survival of all higher trophic level organisms and are integral to optimal ecosystem function. The identification of a novel and significant source of EFA, represented by trematode cercariae, when our current resources are under threat, is essential to not only the maintence of our ecosystem's stability, but also to the preservation of aquatic food webs that provide vital ecosystem services. 


\section{APPENDIX}

Table A.1. Fatty acid composition of the Ribeiroia ondatrae cercariae sample in DW, WW and proportional (\% of total identified FA). FA results are the aggregate for $\sim 30,000$ cercariae.

\begin{tabular}{|c|c|c|c|c|}
\hline \multirow[b]{2}{*}{ Fatty acids } & \multirow[b]{2}{*}{ Mass Fraction $(\mu \mathrm{g} / \mathrm{mg})$ DW } & \multirow[b]{2}{*}{ Mass Fraction $(\mu \mathrm{g} / \mathrm{mg}) \mathrm{WW}$} & \multirow[b]{2}{*}{ Proportional (\% of total FA) } & \multirow[b]{2}{*}{ Mass Fraction (ng/cercariae) DW } \\
\hline & & & & \\
\hline $14: 0$ & 0.77 & 0.43 & 1.88 & 0.28 \\
\hline $14: 1 n-5$ & 0.31 & 0.17 & 0.76 & 0.11 \\
\hline 15:0i & - & - & - & - \\
\hline 15:0a & - & - & - & - \\
\hline 15:0 & 0.80 & 0.44 & 1.95 & 0.29 \\
\hline $15: 1 n-5$ & 0.02 & 0.01 & 0.06 & 0.00 \\
\hline $16: 0$ & 7.78 & 4.31 & 19.02 & 2.85 \\
\hline $16: 1 n-9$ & - & - & - & - \\
\hline $16: 1 n-7 c$ & 1.09 & 0.60 & 2.67 & 0.40 \\
\hline $16: 1 n-7 t$ & 0.98 & 0.54 & 2.39 & 0.36 \\
\hline $17: 0$ & 0.69 & 0.38 & 1.68 & 0.25 \\
\hline $17: 1 n-7$ & 0.03 & 0.02 & 0.07 & 0.01 \\
\hline 18:0 & 5.56 & 3.08 & 13.59 & 2.04 \\
\hline $18: 1 n-9 c$ & 2.51 & 1.39 & 6.15 & 0.92 \\
\hline $18: 1 n-9 t$ & 0.05 & 0.03 & 0.12 & 0.02 \\
\hline $18: 1 n-12 c$ & - & - & - & - \\
\hline $18: 1 n-7 c$ & 1.56 & 0.86 & 3.80 & 0.57 \\
\hline $18: 1 n-7 t$ & 0.63 & 0.35 & 1.53 & 0.23 \\
\hline 19:0 & 0.17 & 0.09 & 0.41 & 0.06 \\
\hline $19: 1 \mathrm{n}-12$ & 0.10 & 0.06 & 0.25 & 0.04 \\
\hline $18: 2 n-6 c($ LNA $)$ & 1.86 & 1.03 & 4.55 & 0.68 \\
\hline $20: 0$ & 0.48 & 0.26 & 1.16 & 0.17 \\
\hline $18: 3 n-6$ & - & - & - & - \\
\hline $20: 1 \mathrm{n}-15$ & 0.02 & 0.01 & 0.04 & 0.01 \\
\hline $20: 1 \mathrm{n}-12$ & - & - & - & - \\
\hline $20: 1 n-9$ & 0.54 & 0.30 & 1.32 & 0.20 \\
\hline $18: 3 n-3$ (ALA) & 5.02 & 2.78 & 12.26 & 1.84 \\
\hline 19:0D & - & - & - & - \\
\hline $20: 1 n-11$ & - & - & - & - \\
\hline $20: 2 n-6$ & 0.67 & 0.37 & 1.63 & 0.24 \\
\hline $22: 3 n-3$ & 1.12 & 0.62 & 2.74 & 0.41 \\
\hline 22:0 & 0.55 & 0.31 & 1.35 & 0.20 \\
\hline $20: 3 n-6$ & 0.60 & 0.33 & 1.46 & 0.22 \\
\hline 22:1n-11 & - & - & - & - \\
\hline 22:1n-9 & 0.08 & 0.04 & 0.19 & 0.03 \\
\hline $20: 3 n-3$ & 2.78 & 1.54 & 6.80 & 1.02 \\
\hline $20: 4 n-6$ (ARA) & 0.68 & 0.38 & 1.66 & 0.25 \\
\hline $22: 2 n-6$ & 0.05 & 0.03 & 0.12 & 0.02 \\
\hline 24:0 & 1.12 & 0.62 & 2.74 & 0.41 \\
\hline $20: 5 n-3$ (EPA) & 2.16 & 1.20 & 5.27 & 0.79 \\
\hline $24: 1 n-9$ & 0.05 & 0.03 & 0.12 & 0.02 \\
\hline $22: 4 n-6$ & 0.05 & 0.03 & 0.13 & 0.02 \\
\hline $22: 5 n-6$ & - & - & - & - \\
\hline $22: 5 n-3$ & 0.01 & 0.01 & 0.03 & 0.00 \\
\hline $22: 6 n-3$ (DHA) & 0.04 & 0.02 & 0.09 & 0.01 \\
\hline $18: 4 n-3$ & - & - & - & - \\
\hline \multicolumn{5}{|l|}{$\Sigma$} \\
\hline Sum SFA & 17.91 & 9.93 & 43.77 & 6.57 \\
\hline Sum MUFA & 9.09 & 5.04 & 22.23 & 3.33 \\
\hline Sum PUFA & 13.91 & 7.71 & 34.00 & 5.10 \\
\hline Sum MUFA $\geq 18$ & 6.66 & 3.69 & 16.27 & 2.44 \\
\hline Sum MUFA $>18$ & 1.91 & 1.06 & 4.67 & 0.70 \\
\hline Sum C18 PUFA & 6.88 & 3.81 & 16.82 & 2.52 \\
\hline Sum C20 PUFA & 6.88 & 3.82 & 16.82 & 2.52 \\
\hline Sum C22 PUFA & 0.15 & 0.08 & 0.36 & 0.05 \\
\hline Sum EPA/DHA & 2.19 & 1.22 & 5.36 & 0.80 \\
\hline Sum n-6 & 3.90 & 2.17 & 9.55 & 1.43 \\
\hline Sum n-3 & 10.00 & 5.55 & 24.46 & 3.67 \\
\hline Sum Odd chain & 4.40 & 2.44 & 10.76 & 1.61 \\
\hline$n-3 / n-6$ & 2.56 & 2.56 & 2.56 & 0.09 \\
\hline Total & 40.91 & 22.68 & 100.00 & 15.00 \\
\hline
\end{tabular}

LNA: linolenic acid; ALA: alpha-linolenic acid; ARA: arachidonic acid; EPA: eicosapentaenoic acid; DHA: docosahexaenoic acid 
Table A.2. Mean proportional fatty acid composition of Leucorrhinia intacta larvae fed two diet treatments (Mean \pm S.D.).

\begin{tabular}{|c|c|c|c|}
\hline Diet Treatment & SFA (\%) & MUFA (\%) & PUFA (\%) \\
\hline Daphnia spp. & $21.3 \pm 4.90$ & $18.2 \pm 2.07$ & $60.5 \pm 6.19$ \\
\hline Ribeiroia ondatrae & $22.4 \pm 3.44$ & $18.1 \pm 3.06$ & $59.5 \pm 5.38$ \\
\hline $\mathrm{t} / \mathrm{F}$-value, $\mathrm{df}$ & $\mathrm{t}=-3.97,37$ & $\mathrm{~F}=1.20,36$ & $\mathrm{~F}=0.01,36$ \\
\hline P-value & 0.0003 & 0.280 & 0.935 \\
\hline
\end{tabular}

Table A.3. Mean proportional essential fatty acid composition of Leucorrhinia intacta larvae fed two diet treatments (Mean \pm S.D.).

\begin{tabular}{|c|c|c|c|c|c|}
\hline Diet Treatment & ALA & LNA & ARA & EPA & DHA \\
\hline Daphnia spp. & $15.4 \pm 2.91$ & $8.0 \pm 1.17$ & $2.8 \pm 0.445$ & $30.2 \pm 4.24$ & $0.010 \pm 0.019$ \\
\hline Ribeiroia ondatrae & $14.5 \pm 4.53$ & $7.2 \pm 2.07$ & $2.7 \pm 0.882$ & $30.5 \pm 8.44$ & $0.004 \pm 0.003$ \\
\hline
\end{tabular}

Table A.4. Absolute ( $\mu \mathrm{g}$ of FA/mg DW) fatty acid composition of Leucorrhinia intacta larvae fed two diet treatments (Mean \pm S.D.).

\begin{tabular}{|c|c|c|c|}
\hline Diet Treatment & SFA & MUFA & PUFA \\
\hline Daphnia spp. & $3.53 \pm 2.07$ & $2.98 \pm 1.52$ & $10.2 \pm 6.35$ \\
\hline Ribeiroia ondatrae & $5.34 \pm 4.62$ & $4.08 \pm 3.20$ & $14.4 \pm 14.2$ \\
\hline
\end{tabular}

Table A.5. Absolute ( $\mu \mathrm{g}$ of FA/mg dry weight) essential fatty acid composition of Leucorrhinia intacta larvae fed two diet treatments (Mean \pm S.D.).

\begin{tabular}{|c|c|c|c|c|c|}
\hline Diet Treatment & ALA & LNA & ARA & EPA & DHA \\
\hline Daphnia spp. & $2.61 \pm 1.65$ & $1.37 \pm 0.902$ & $0.474 \pm 0.291$ & $5.10 \pm 3.30$ & $0.001 \pm 0.002$ \\
\hline Ribeiroia ondatrae & $3.56 \pm 3.80$ & $1.80 \pm 1.64$ & $0.762 \pm 0.952$ & $7.16 \pm 6.76$ & $0.001 \pm 0.002$ \\
\hline
\end{tabular}




\section{REFERENCES}

Ahlgren, G., Vrede, T., and Goedkoop, W. (2009). Fatty acid ratios in freshwater fish, zooplankton and zoobenthos-are there specific optima? In Lipids in aquatic ecosystems (pp. 147-178). Springer, New York, NY.

Alonzo, F., and Mayzaud, P. (1999). Spectrofluorometric quantification of neutral and polar lipids in zooplankton using Nile red. Marine Chemistry, 67, 289-301.

Anderson, R. M., Mercer, J. G., Wilson, R. A. and Carter, N. P. (1982). Transmission of Schistosoma mansoni from man to snail: experimental studies of miracidial survival and infectivity in relation to larval age, water temperature, host size and host age. Parasitology, 85, 339-360.

Arts, M. T., Ackman, R. G., and Holub, B. J. (2001). " Essential fatty acids" in aquatic ecosystems: a crucial link between diet and human health and evolution. Canadian Journal of Fisheries and Aquatic Sciences, 58, 122-137.

Arts, M. T., and Evans, M. S. (1991). Optical-digital measurements of energy reserves in Calanoid copepods: Intersegmental distribution and seasonal patterns. Limnology and Oceanography, 36, 289-298.

Arts, M. T., and Kohler, C. C. (2009). Health and condition in fish: the influence of lipids on membrane competency and immune response. In Lipids in aquatic ecosystems (pp. 237256). Springer, New York, NY.

Arts, M. T., and Sprules, W. G. (1987). Energy reserves of three zooplankton species from two lakes with different metal concentrations. Canadian Journal of Fisheries and Aquatic Sciences, 44, 458-466.

Arts, M. T., and Sprules, W. G. (1988). Evidence for indirect effects of fish predation on maternal lipid investment in Holopedium gibberum. Canadian Journal of Fisheries and Aquatic Sciences, 45, 2147-2155.

Arts, M. T., Brett, M. T., and Kainz, M. (Eds.). (2009). Lipids in Aquatic Ecosystems. Springer Science and Business Media.

Arts, M.T., and B.C. Wainman (eds.). (1999). Lipids in Freshwater Ecosystems. Springer, New York, USA. 319 pgs. ISBN 978-0-387-98505-3.

Båmstedt, U. (1988). Ecological significance of individual variability in copepod bioenergetics. Hydrobiologia, 167, 43-59. 
Bandstra, S. R., Fried, B., and Sherma, J. (2006). High-performance thin-layer chromatographic analysis of neutral lipids and phospholipids in Biomphalaria glabrata patently infected with Echinostoma caproni. Parasitology Research, 99, 414-418.

Bar-On, Y. M., Phillips, R., and Milo, R. (2018). The biomass distribution on Earth. Proceedings of the National Academy of Science. DOI: 10.1073/pnas. 1711842115

Bascompte, J. (2009) Disentangling the web of life. Science, 325, 416-419.

Baudoin, M. (1975). Host castration as a parasitic strategy. Evolution, 29, 335-352.

Baxter, C. V., Fausch, K. D., and Carl Saunders, W. (2005). Tangled webs: reciprocal flows of invertebrate prey link streams and riparian zones. Freshwater Biology, 50, 201-220.

Bazan N. G. (2009). Cellular and molecular events mediated by docosahexaenoic acid derived neuroprotectin D1 signaling in photoreceptor cell survival and brain protection. Prostaglandins, Leukotrienes and Essential Fatty Acids, 81, 205-11.

Bernot, R. J. (2003). Trematode infection alters the antipredator behavior of a pulmonate snail. Journal of the North American Benthological Society, 22, 241-248.

Bernot, R. J., and Lamberti, G. A. (2008). Indirect effects of a parasite on a benthic community: an experiment with trematodes, snails and periphyton. Freshwater Biology, 53, 322-329.

Bernot, R. J., and Turner, A. M. (2001). Predator identity and trait-mediated indirect effects in a littoral food web. Oecologia, 129, 139-146.

Bogdan, K. G., and Gilbert, J. J. (1984). Body size and food size in freshwater zooplankton. Proceedings of the National Academy of Sciences, 81, 6427-6431.

Boissonnot, L., Niehoff, B., Hagen, W., Søreide, J. E., and Graeve, M. (2016). Lipid turnover reflects life-cycle strategies of small-sized Arctic copepods. Journal of Plankton Research, 38, 1420-1432.

Brenna, J. T., Salem, N., Sinclair, A. J., and Cunnane, S. C. (2009). $\alpha$-Linolenic acid supplementation and conversion to n-3 long-chain polyunsaturated fatty acids in humans. Prostaglandins, Leukotrienes and Essential Fatty Acids, 80, 85-91.

Brett, M. T., Müller-Navarra, D. C., Ballantyne, A. P., Ravet, J. L., and Goldman, C. R. (2006b). Daphnia fatty acid composition reflects that of their diet. Limnology and Oceanography, 51, 2428-2437.

Brett, M. T., Müller-Navarra, D., Ballantyne, A., Ravet, J., and Goldman, C. (2006a). The nutritional importance of poly-unsaturated fatty acids and their use as trophic markers for herbivorous zooplankton: does it contradict? Archiv für Hydrobiologie, 164, 501-513. 
Brett, M., and Müller-Navarra, D. (1997). The role of highly unsaturated fatty acids in aquatic food web processes. Freshwater Biology, 38, 483-499.

Buckner, J. S., and Hagen, M. M. (2003). Triacylglycerol and phospholipid fatty acids of the silverleaf whitefly: composition and biosynthesis. Archives of Insect Biochemistry and Physiology, 53, 66-79.

Burdon, F. J., and Harding, J. S. (2008). The linkage between riparian predators and aquatic insects across a stream-resource spectrum. Freshwater Biology, 53, 330-346.

Burns, C. W. (1992). Population dynamics of crustacean zooplankton in a mesotrophic lake, with emphasis on Boeckella hamata BREHM (Copepoda: Calanoida). International Review of Hydrobiology, 77, 553-577.

Burns, C. W., Brett, M. T., and Schallenberg, M. (2011). A comparison of the trophic transfer of fatty acids in freshwater plankton by cladocerans and calanoid copepods. Freshwater Biology, 56, 889-903.

Byers, J. (2009). Including parasites in food webs. Trends in Parasitology, 25, 55-57.

Calder, P. (2010). Omega-3 fatty acids and inflammatory processes. Nutrients, 2, 355-374.

Carman, K. R., Thistle, D., Ertman, S. C., and Foy, M., (1991). Nile red as a probe for lipidstorage products in benthic copepods. Marine Ecology Progress Series, 74, 307-311.

Catania, S. V., Koprivnikar, J., and McCauley, S. J. (2016). Size-dependent predation alters interactions between parasites and predators. Canadian Journal of Zoology, 94, 631-635.

Cattin, M. F., Bersier, L. F., Banašek-Richter, C., Baltensperger, R., and Gabriel, J. P. (2004). Phylogenetic constraints and adaptation explain food-web structure. Nature, 427, 835839.

Chen, M., Liu, H., and Chen, B. (2012). Effects of dietary essential fatty acids on reproduction rates of a subtropical calanoid copepod, Acartia erythraea. Marine Ecology Progress Series, 455, 95-110.

Chen, W., Zhang, C., Song, L., Sommerfeld, M., Hu, Q. (2009). A high throughput Nile red method for quantitative measurement of neutral lipids in microalgae. Journal of Microbiological Methods., 77, 41-7.

Chiang, C. P., and Caulfield, J. P. (1989). Human lipoprotein binding to schistosomula of Schistosoma mansoni: displacement by polyanions, parasite antigen masking, and persistence in young larvae. American Journal of Pathology, 135, 1015-1024. 
Choubisa, S. L. (2008). Mode of nutrition in pathogenic trematode larvae (redia and cercaria) which infect hepatopancreas of fresh water snails (Mollusca: Gastropoda). Journal of Parasitic Diseases, 32, 68-73.

Christie W. W. (1989). The analysis of fatty acids. In Gas Chromatography and Lipids. PJ Barnes and Associates (The Oily Press), Bridgewater, UK.

Cohen, J. E., Luczak, T., Newman, C. M., and Zhou, Z. M. (1990). Stochastic structure and nonlinear dynamics of food webs: qualitative stability in a Lotka-Volterra cascade model. Proceedings of the Royal Society of London B: Biological Sciences, 240, 607-627.

Collier, K. J., Bury, S., and Gibbs, M. (2002). A stable isotope study of linkages between stream and terrestrial food webs through spider predation. Freshwater Biology, 47, 1651-1659.

Combes, C., Fournier, A., Moné, H. and Théron, A. (1994). Behaviours in trematode cercariae that enhance parasite transmission: patterns and processes. Parasitology, 109, S3-S13.

Connor, W. E. (2000). Importance of n-3 fatty acids in health and disease. The American Journal of Clinical Nutrition, 71, 171-175.

Cooksey, K. E., Guckert, J. B., Williams, S. A., Callis, P. R. (1987). Fluorometric determination of the neutral lipid content of microalgal cells using Nile Red. Journal of Microbiological Methods, 6, 333-345.

Cort, W. W. (1914). Larval trematodes from North American fresh-water snails. Journal of Parasitology, 1, 65-84.

Cort, W. W., Ameel, D. J., Van der Woude, A. (1954). Germinal development in sporocysts and rediae of the digenetic trematodes. Experimental Parasitology, 3, 185-225.

Cort, W. W., and Brackett, S. (1937). Identification of strigeid cercariae by differences in their behaviour during free life. The Journal of Parasitology, 23, 297-299.

Crews, A. E., and Yoshino, T. P. (1989). Schistosoma mansoni: effect of infection on reproduction and gonadal growth in Biomphalaria glabrata. Experimental Parasitology, 68, 326-334.

Dalsgaard, J., John, M. S., Kattner, G., Müller-Navarra, D., and Hagen, W. (2003). Fatty acid trophic markers in the pelagic marine environment. Advances in Marine Biology, 46, 225-340.

Danielsdottir, M. G., Brett, M. T., and Arhonditsis, G. B. (2007). Phytoplankton food quality control of planktonic food web processes. Hydrobiologia, 589, 29-41.

de Ruiter, P. C., Wolters, V., Moore, J. C., and Winemiller, K. O. (2005). Food web ecology: playing Jenga and beyond. Science, 309, 68-71. 
Dembitsky, V. M., Kashin, A. G., and Stefanov, K. (1992). Comparative investigation of phospholipids and fatty acids of freshwater molluscs from the Volga river basin. Comparative Biochemistry and Physiology Part B: Comparative Biochemistry, 102, 193-198.

Demott, W., and Müller-Navarra, D. (1997). The importance of highly unsaturated fatty acids in zooplankton nutrition: evidence from experiments with Daphnia, a cyanobacterium and lipid emulsions. Freshwater Biology, 38, 649-664.

Deschaseaux, E., Taylor, A., Maher, W., Davis, A. (2010). Cellular responses of encapsulated gastropod embryos to multiple stressors associated with climate change. Journal of Experimental Marine Biology and Ecology, 383, 130-136

Desvilettes, C. H., Bourdier, G., Amblard, C. H., and Barth, B. (1997). Use of fatty acids for the assessment of zooplankton grazing on bacteria, protozoans and microalgae. Freshwater Biology, 38, 629-637.

Diconza, J. J., and Basch, P. F. (1975). Histochemical demonstration of acetylcholinesterase in sporocysts of Schistosoma mansoni (Trematoda). Parasitology, 71, 305-310.

Diconza, J. J., and Basch, P. F. (1976). Accumulation of lipids in Schistosoma mansoni sporocysts cultured in vitro. Journal of Invertebrate Pathology, 28, 337-340.

Dobson, A., Lafferty, K.D., Kuris, A.M., Hechinger, R.F., Jetz, W. (2008). Homage to Linnaeus: How many parasites? How many hosts? Proceedings of the National Academy of Sciences, $105,11482-11489$.

Douglas R. Tocher. (2003). Metabolism and Functions of Lipids and Fatty Acids in Teleost Fish. Reviews in Fisheries Science, 11, 107-184.

Dumont, H. J., Van de Velde, I., and Dumont, S. (1975). The dry weight estimate of biomass in a selection of Cladocera, Copepoda and Rotifera from the plankton, periphyton and benthos of continental waters. Oecologia, 19, 75-97.

Dunne, J. A., Lafferty, K. D., Dobson, A. P., Hechinger, R. F., Kuris, A. M., Martinez, N. D., Stouffer, D. B., Thieltges, D.B., Williams, R.J., Zander, C.D. (2013). Parasites affect food web structure primarily through increased diversity and complexity. PLoS Biology, 11, e1001579.

Ebert, D. (2005). Ecology, Epidemiology, and Evolution of Parasitism in Daphnia. National Library of Medicine. 110 pgs.

Edgecombe, G. D., Giribet, G., Dunn, C. W., Hejnol, A., Kristensen, R. M., Neves, R. C., Rouse, G. W., Worsaae, K., Sorensen, M. V. (2011). Higher-level metazoan relationships: recent progress and remaining questions. Organisms Diversity and Evolution, 11, 151-172. 
Elton, C. (1927). Animal Ecology. London, Sidgwick and Jackson.

Erasmus, D. A. (1972). The Biology of Trematodes. Printed in Great the Britain at the University Press, Belfast.

Farooqui, A. A. (2009). Lipid mediators in the neural cell nucleus: their metabolism, signaling, and association with neurological disorders. The Neuroscientist, 15, 392-407.

Faust, E. C. (1919). The excretory system in digenea: II: Observations on the excretory system in distome cercariae. The Biological Bulletin, 36, 322-339.

Folch, J., Lees, M., and Sloane-Stanley, G.H. (1957). A simple method for the isolation and purification of total lipides from animal tissues. The Journal of Biological Chemistry, 226, 497-509.

Frandsen, F., and Christensen, N. O. (1984). An introductory guide to the identification of cercariae from African freshwater snails with special reference to cercariae of trematode species of medical and veterinary importance. Acta Tropica, 41, 181-202.

Fried, B., and Morrone, L. J. (1970). Histochemical lipid studies on Echinostoma revolutum. Proceedings of the Helminthological Society of Washington, 37, 122-123.

Fried, B., and Pucci, D. L. (1976). Histochemical and thin layer chromatographic analyses of neutral lipids in Leucochloridiomorpha constantiae (Trematoda) adults. International Journal for Parasitology, 6, 479-482.

Fried, B., and Sherma, J. (1990). Thin layer chromatography of lipids found in snails. Journal of Planar Chromatography - Modern TLC, 3, 290 -299.

Fried, B., and Toledo, R. (Eds.). (2009). The Biology of Echinostomes: From the Molecule to the Community (Vol. 9). Springer Science and Business Media.

Fried, B., Eyster, L. S., and Pechenik, J. A. (1998c). Histochemical glycogen and neutral lipid in Echinostoma trivolvis cercariae and effects of exogenous glucose on cercarial longevity. Journal of Helminthology, 72, 83-85.

Fried, B., Frazer, B. A., and Kanev, I. (1998a). Comparative observations on cercariae and metacercariae of Echinostoma trivolvis and Echinoparyphium sp. The Journal of Parasitology, 623-626.

Fried, B., Frazer, B. A., Lee, M. S., and Sherma, J. (1998b). Thin-layer chromatography and histochemistry analyses of neutral lipids in Helisoma trivolvis infected with four species of larval trematodes. Parasitology Research, 84, 369-373. 
Fried, B., Holender, E. S., Shetty, P. H., and Sherma, J. (1990). Effects of Echinostoma trivolvis (Trematoda) infection on neutral lipids, sterols, and carotenoids in Helisoma trivolvis (Gastropoda). Comparative Biochemistry and Physiology Part B: Comparative Biochemistry, 97, 601-604.

Fried, B., Muller, E. E., Broadway, A., and Sherma, J. (2001). Effects of diet on the development of Schistosoma mansoni in Biomphalaria glabrata and on the neutral lipid content of the digestive gland-gonad complex of the snail. Journal of Parasitology, 87, 223-225.

Fried, B., Rao, K. S., and Sherma, J. (1992b) Fatty acid composition of Biomphalaria glabrata (Gastropoda: Planorbidae) fed hen's egg yolk versus leaf lettuce. Comparative Biochemistry and Physiology, 101, 351-352.

Fried, B., Rao, K. S., Sherma, J., and Huffman, J. E. (1993b). Fatty acid composition of Echinostoma trivolvis (Trematoda) rediae and adults and of the digestive gland-gonad complex of Helisoma trivolvis (Gastropoda) infected with the intramolluscan stages of this echinostome. Parasitology Research, 79, 471-474.

Fried, B., Rao, K. S., Sherma, J., Huffman, J. E. (1993c). Fatty acid composition of Goniobasis virginica Physa sp. and Viviparus malleatus (Mollusca: Gastropoda) from Lake Musconetcong, New Jersey. Biochemical Systematics and Ecology, 21, 809-812.

Fried, B., Rao, S., Shermas, J. (1992a). Fatty acid composition of two strains of Helisoma trivolvis (Gastropoda). Biochemical Systematics and Ecology, 20, 553-557.

Fried, B., Schafer, S., and Kim, S. (1989). Effects of Echinostoma caproni infection on the lipid composition of Biomphalaria glabrata. International Journal for Parasitology, 19, 353354.

Fried, B., Sherma, J., Rao, K. S., and Ackman, R. G. (1993a). Fatty acid composition of Biomphalaria glabrata (Gastropoda: Planorbidae) experimentally infected with the intramolluscan stages of Echinostoma caproni (Trematoda). Comparative Biochemistry and Physiology Part B: Comparative Biochemistry, 104, 595-598.

Fritz, K. A., Kirschman, L. J., McCay, S. D., Trushenski, J. T., Warne, R. W., Whiles M. R. (2017). Subsidies of essential nutrients from aquatic environments with immune function in terrestrial consumers. Freshwater Science, 36, 893-900.

Furlong, S. T. (1991). Unique roles for lipids in Schistosoma mansoni. Parasitology Today, 7 , 59-62.

Furlong, S. T., and Caulfield, J. P. (1989). Schistosoma mansoni: synthesis and release of phospholipids, lysophospholipids, and neutral lipids by schistosomula. Experimental Parasitology, 69, 65-77. 
Furlong, S. T., Thibault, K. S., and Rogers, R. A. (1992). Fluorescent phospholipids preferentially accumulate in sub-tegumental cells of schistosomula of Schistosoma mansoni. Journal of Cell Science, 103(3), 823-830.

Furlong, S. T., Thibault, K. S., Morbelli, L. M., Quinn, J. J., and Rogers, R. A. (1995). Uptake and compartmentalization of fluorescent lipid analogs in larval Schistosoma mansoni. Journal of Lipid Research, 36, 1-12.

Fusco, A. C., Salafsky, B., and Delbrook, K. (1986). Schistosoma mansoni: production of cercarial eicosanoids as correlates of penetration and transformation. The Journal of Parasitology, 397-404.

Fusco, A. C., Salafsky, B., and Kevin, M. B. (1985). Schistosoma mansoni: eicosanoid production by cercariae. Experimental Parasitology, 59, 44-50.

Galaktionov, K. V., and Dobrovolskij, A. (2013). The biology and evolution of trematodes: an essay on the biology, morphology, life cycles, transmissions, and evolution of digenetic trematodes. Springer Science and Business Media.

Gallager, S. M., and Mann, R. (1986). Individual variability in lipid content of bivalve larvae quantified histochemically by absorption photometry. Journal of Plankton Research, 8 , 927-937.

Galloway, A. W. E., and Winder, M. (2015). Partitioning the relative importance of phylogeny and environmental conditions on phytoplankton fatty acids. Plos One, 10: e0130053.

Georgieva, S., Selbach, C., Faltýnková, A., Soldánová, M., Sures, B., Skírnisson, K., and Kostadinova, A. (2013). New cryptic species of the 'revolutum' group of Echinostoma (Digenea: Echinostomatidae) revealed by molecular and morphological data. Parasites and Vectors, 6, 64.

Ginetskinskaya, T. A. (1988). Trematodes: Their Life Cycles, Biology and Evolution. New Delhi: Amerind Publishing Company.

Ginger, C. D., and Fairbairn, D. (1966). Lipid metabolism in helminth parasites. II. The major origins of the lipids of Hymenolepis diminuta (Cestoda). The Journal of Parasitology, 1097-1107.

Gladyshev, M. I., Arts, M. T., and Sushchik, N. I. (2009). Preliminary estimates of the export of omega-3 highly unsaturated fatty acids (EPA+DHA) from aquatic to terrestrial ecosystems. In Lipids in Aquatic Ecosystems (pp. 179-210). Springer, New York, NY.

Gladyshev, M. I., Sushchik, N. N., and Makhutova, O. N. (2013). Production of EPA and DHA in aquatic ecosystems and their transfer to the land. Prostaglandins and Other Lipid Mediators, 107, 117-126. 
Gladyshev, M. I., Sushchik, N. N., Makhutova, O. N., Kalacheva, G. S., Kolmakova, A. A., Kravchuk, E. S., Dubovskaya, O. P. (2008). Efficiency of transfer of essential polyunsaturated fatty acids along trophic chains in aquatic ecosystems. Biochemistry, Biophysics, and Molecular Biology, 426, 158-160.

Goulden, C. E., and Hornig, L. L. (1980). Population oscillations and energy reserves in planktonic cladocera and their consequences to competition. Proceedings of the National Academy of Sciences, 77, 1716-1720.

Goulden, C. E., Henry, L. L., and Tessier, A. J. (1982). Body size, energy reserves, and competitive ability in three species of Cladocera. Ecology, 63, 1780-1789.

Graeve, M., Albers, C., and Kattner, G. (2005). Assimilation and biosynthesis of lipids in arctic Calanus species based on feeding experiments with a $13 \mathrm{C}$ labelled diatom. Journal of Experimental Marine Biology and Ecology, 317, 109-125.

Gratton, C., and Zanden, M. (2009). Flux of aquatic insect productivity to land: comparison of lentic and lotic ecosystems. Ecology, 90, 2689-2699.

Green, J. (1957). Carotenoids in Daphnia. Proceedings of the Royal Society of London, 147, 392401.

Gulati, R., and Demott, W. (1997). The role of food quality for zooplankton: remarks on the state-of-the-art, perspectives and priorities. Freshwater Biology, 38, 753-768.

Gupta, A. N., Guraya, S. S., and Sharma, P. N. (1974). Histochemical observations on the excretory system of digenetic trematodes. Acta morphologica NeerlandoScandinavica, 12, 231-242.

Haas, W. (1994). Physiological analyses of host-finding behaviour in trematode cercariae: Adaptations for transmission success. Parasitology, 109, 15-29.

Haas, W., Beran, B., Loy, C. (2008). Selection of the host's habitat by cercariae: from laboratory experiments to the field. The Journal of Parasitology, 94, 1233-1238.

Haas, W., Diekhoff, D., Koch, K., Schmalfuss, G., Loy, C. (1997). Schistosoma mansoni cercariae: stimulation of acetabular gland secretion is adapted to the chemical composition of mammalian skin. The Journal of Parasitology, 83, 1079-1085.

Hairston, N.G., Jr. and Hairston, N.G. Sr. (1993). Cause-effect relationships in energy flow, trophic structure, and interspecific interactions. American Society of Naturalists, 142, $379-411$.

Hechinger, R.F., (2013). A metabolic and body-size scaling framework for parasite within-host abundance, biomass, and energy flux. The American Naturalist, 182, 234-248. 
Hechinger, R.F., Lafferty, K.D., Dobson, A.P., Brown, J.H., Kuris, A.M. (2011). A common scaling rule for abundance, energetics, and production of parasitic and free-living species. Science, 333, 445-448.

Held, P., and Raymond, K. (2011). Determination of algal cell lipids using Nile Red-using microplates to monitor neutral lipids in Chlorella Vulgaris. Biotek Application Note, www.biotek.com.

Hentschel, B. T., 1998. Spectrofluorometric quantification of neutral and polar lipids suggests a food-related recruitment bottleneck for juveniles of a deposit-feeding polychaete population. Limnology and Oceanography., 43, 543-549.

Hernandez, A. D., and Sukhdeo, M. V. (2008). Parasites alter the topology of a stream food web across seasons. Oecologia, 156, 613-624.

Hill, W. R., Rinchard, J., and Czesny, S. (2011). Light, nutrients and the fatty acid composition of stream periphyton. Freshwater Biology, 56, 1825-1836.

Hiltunen, M., Honkanen, M., Taipale, S., Strandberg, U., Kankaala, P. (2017). Trophic upgrading via the microbial food web may link terrestrial dissolved organic matter to Daphnia. Journal of Plankton Research, 39, 861-869.

Hixson, S. M., Sharma, B., Kainz, M. J., Wacker, A., and Arts, M. T. (2015). Production, distribution, and abundance of long-chain omega-3 polyunsaturated fatty acids: a fundamental dichotomy between freshwater and terrestrial ecosystems. Environmental Reviews, 23, 414-424.

Hoffman, D., Boettcher, J., and Diersen-Schade, D. (2009). Toward optimizing vision and cognition in term infants by dietary docosahexaenoic and arachidonic acid supplementation: A review of randomized controlled trails. Prostaglandins, Leukotrienes and Essential Fatty Acids, 81, 151-158.

Holm, N. P., and Shapiro, J. (1984). An examination of lipid reserves and the nutritional status of Daphnia pulex fed Aphanizomenon flos-aquae. Limnology and Oceanography, 29, 11371140.

Hussey, K. L. (1943). Further studies on the comparative embryological development of the excretory system in digenetic trematodes. Transactions of the American Microscopical Society, 62, 271-279.

Huxham, M., Raffaelli, D., and Pike, A. (1995). Parasites and food web patterns. Journal of Animal Ecology, 168-176.

Innis, S. M. (2007). Dietary (n-3) fatty acids and brain development. The Journal of Nutrition, 137, 855-859. 
Jacobsen, N. S., and Fairbairn, D. (1967). Lipid metabolism in helminth parasites III. Biosynthesis and interconversion of fatty acids by Hymenolepis diminuta (Cestoda). The Journal of Parasitology, 355-361.

Johnson, P. T. J., Lunde, K. B., Thurman, E. M., Ritchie, E. G., Wray, S. N., Sutherland, D. R., Kapfer, J. M., Frest, T. J., Bowerman, J., and Blaustein, A. R. (2002). Parasite (Ribeiroia ondatrae) infection linked to amphibian malformations in the western United States. Ecological Monographs, 72, 151-168.

Johnson, P.T. and McKenzie, V.J., 2009. Effects of environmental change on helminth infections in amphibians: exploring the emergence of Ribeiroia and Echinostoma infections in North America. In The biology of echinostomes (pp. 249-280). Springer, New York, NY.

Johnson, P. T. J., Sutherland, D. R., Kinsella, J. M., and Lunde, K. B. (2004). Review of the trematode genus Ribeiroia (Psilostomidae): Ecology, life history, and pathogenesis with special emphasis on the amphibian malformation problem. Advances in Parasitology, 57, $191-253$.

Johnson, P. T., and Chase, J. M. (2004). Parasites in the food web: linking amphibian malformations and aquatic eutrophication. Ecology Letters, 7(7), 521-526.

Johnson, P. T., Dobson, A., Lafferty, K. D., Marcogliese, D. J., Memmott, J., Orlofske, S. A., Poulin, R., and Thieltges, D. W. (2010). When parasites become prey: ecological and epidemiological significance of eating parasites. Trends in Ecology and Evolution, 25, 362-371.

Jones, D. A., Kanazawa, A., and Ono, K. (1979). Studies on the nutritional requirements of the larval stages of Penaeus japonicus using microencapsulated diets. Marine Biology, 54, 261-267.

Joseph, J. D. (1982). Lipid composition of marine and estuarine invertebrates. Part II: Mollusca. Progress in Lipid Research, 21, 109-153.

Kabeya, N., Fonseca, M. M., Ferrier, D. E. K., Navarro, J. C., Bay, L. K., Francis, D. S., Tocher, D. R., Castro, L. F., Monroig, O. (2018). Genes for de novo synthesis of omega-3 polyunsaturated fatty acids are widespread in animals. Science Advances, 4, 2-8.

Kainz, M., Arts, M. T., and Mazumder, A. (2004). Essential fatty acids in the planktonic food web and their ecological role for higher trophic levels. Limnology and Oceanography, 49, 1784-1793.

Kanazawa, A., Teshima, S. I., and Sakamoto, M. (1985). Effects of dietary lipids, fatty acids, and phospholipids on growth and survival of prawn (Penaeus japonicus)

larvae. Aquaculture, 50, 39-49. 
Kaplan, A. T., Rebhal, S., Lafferty, K. D., and Kuris, A. M. (2009). Small estuarine fishes feed on large trematode cercariae: lab and field investigations. Journal of Parasitology, 95, 477-480.

Kato, C., Iwata, T., and Wada, E. (2004). Prey use by web-building spiders: stable isotope analyses of trophic flow at a forest-stream ecotone. Ecological Research, 19, 633-643.

Kautza, A., and Sullivan, S. M. P. (2016). The energetic contributions of aquatic primary producers to terrestrial food webs in a mid-size river system. Ecology, 97, 694-705.

Kimura, K., Yamaoka, M., and Kamisaka, Y. (2004). Rapid estimation of lipids in oleaginous fungi and yeasts using Nile red fluorescence. Journal of Microbiological Methods, 56(3), 331-338.

Klobučar G. I. V., Lajtner J., Erben R. (1997). Lipid peroxidation and histopathological changes in the digestive gland of a freshwater snail Planorbarius corneus L. (Gastropoda, Pulmonata) exposed to chronic and sub-chronic concentrations of PCP. Bulletin of Environmental Contamination and Toxicology, 58, 128-134.

Koprivnikar, J., and Poulin, R. (2009). Effects of temperature, salinity, and water level on the emergence of marine cercariae. Parasitology Research, 105, 957-965.

Kris-Etherton, P. M., Harris, W. S., Appel, L. J. (2002). Fish consumption, fish oil, omega-3 fatty acids, and cardiovascular disease. Circulation, 106, 2747-57.

Kuosa, H., and Gyllenberg, G. (1989). Effect of lipid reserves on individual carbon content of two planktonic freshwater copepod species. Hydrobiologia, 185, 245-248.

Kuris, A. M., Hechinger, R. F., Shaw, J. C., Whitney, K. L., Aguirre-Macedo, L., Boch, C. A., Dobson, A. P., Dunham, E. J., Fredensborg, B. L., Huspeni, T. C., Lorda, J., Mababa, L., Mancini, F. T., Mora, A. B., Pickering, M., Talhouk, N. L., Torchin, M. E., and Lafferty, K. D. (2008). Ecosystem energetic implications of parasite and free-living biomass in three estuaries. Nature, 454, 515.

Lafferty, K. D., Allesina, S., Arim, M., Briggs, C. J., De Leo, G., Dobson, A. P., Dunne, J., Johnson, P., Kuris, A., Marcogliese, D., Martinez, N., Memmott, J., Marquet, P., McLaughlin, J., Mordecai, E., Pascual, M., Poulin, R., Thieltges, D. (2008). Parasites in food webs: the ultimate missing links. Ecology Letters, 11, 533-546.

Lafferty, K. D., Dobson, A. P., Kuris, A. M. (2006). Parasites dominate food web links. Proceedings of the National Academy of Sciences, 103, 11211-11216.

Lambden, J., and Johnson, P. T. (2013). Quantifying the biomass of parasites to understand their role in aquatic communities. Ecology and Evolution, 3, 2310-2321. 
Lands, W. (2009). Human life: caught in the food web. In Lipids in Aquatic Ecosystems. Edited by M.T. Arts, M.T. Brett, and M.J. Kainz. Springer, New York. pp. 327-354.

Lau, D. C., Vrede, T., Pickova, J., and Goedkoop, W. (2012). Fatty acid composition of consumers in boreal lakes-variation across species, space and time. Freshwater Biology, 57, 24-38.

Lauritzen, L., Hansen, H. S., Jorgensen, M. H., Michaelsen, K. F. (2001). The essentiality of long chain n-3 fatty acids in relation to development and function of the brain and retina. Progress in Lipid Research, 40, 1-94.

Lawson, R. and Wilson, R. A. (1980). The survival of the cercariae of Schistosoma mansoni in relation to water temperature and glycogen utilization. Parasitology, 81, 337-348.

Lefèvre, T., Lebarbenchon, C., Gauthier-Clerc, M., Misse, D., Poulin, R., and Thomas, F. (2009). The ecological significance of manipulative parasites. Trends in Ecology and Evolution, 24, 41-48.

Leicht, K. (2014). Implications of heat waves on immune defence, life history traits, and adaptive potential: a snail's perspective. Jyväskylä Studies in Biological and Environmental Science, 281. 1456-9701.

Leung, T. L., and Poulin, R. (2011). Small worms, big appetites: ratios of different functional morphs in relation to interspecific competition in trematode parasites. International Journal for Parasitology, 41, 1063-1068.

Levri, E. P. (1999). Parasite-induced change in host behavior of a freshwater snail: parasitic manipulation or by-product of infection? Behavioral Ecology, 10, 234-241.

Levri, E. P., and Lively, C. M. (1996). The effects of size, reproductive condition, and parasitism on foraging behaviour in a freshwater snail, Potamopyrgus antipodarum. Animal Behaviour, 51, 891-901.

Libkind, D., Arts M. T. and Van Broock, M. (2008). Fatty acid composition of cold-adapted carotenogenic basidiomycetous yeasts. Revista Argentina de Microbiología 40, 193-197.

Liddell, C., Welsh, J., van der Meer, J. and Thieltges, D. W. (2017). The effect of dose and frequency of exposure to infectious stages on trematode infection intensity and success in mussels. Diseases of Aquatic Organisms, 125, 85-92.

Lindeman, R. L. (1942). The trophic-dynamic aspect of ecology. Ecology, 23, 399-418.

Lombardo, P., and Cooke, G. D. (2002). Consumption and preference of selected food types by two freshwater gastropod species. Archiv. für. Hydrobiologie, 155, 667-685. 
Lombardo, P., and Cooke, G.D. (2002). Consumption and preference of selected food types by two freshwater gastropod species, Arch Hydrobiology, 155, 667-685

Loreau, M., Roy, J., and Tilman, D. (2005). Linking ecosystem and parasite ecology. Parasitism and Ecosystems, 13.

Lotka, A. J. (1925). Elements of Physical Biology. Baltimore, Williams \& Wilkins.

Loy, C, and Haas, W. (2001). Prevalence of cercariae from Lymnaea stagnalis snails in a pond system in Southern Germany. Parasitology Research, 87, 878-882.

Lunetta, J. E., and Vernberg, W. B. (1971). Fatty acid composition of parasitized and nonparasitized tissue of the mud-flat snail, Nassarius obsolete (Say). Experimental Parasitology, 30, 244-248.

Lustrino, D., Tunholi-Alves, V. M., Tunholi, V. M., Marassi, M. P., and Pinheiro, J. (2010). Lipids analysis in hemolymph of African giant Achatina fulica (Bowdich, 1822) exposed to different photoperiods. Brazilian Journal of Biology, 70, 129-134.

Lyholt, H. C. K. and K. Buchmann, 1996. Diplostomum spathaceum: effects of temperature and light on cercarial shedding and infection of rainbow trout. Diseases of Aquatic Organisms $25,169-173$.

Mandlowitz, S., Dusanic, D., and Lewert, R. M. (1960). Peptidase and lipase activity of extracts of Schistosoma mansoni cercariae. Journal of Parasitology, 46, 89-90.

Marcogliese, D. J. (2002). Food webs and the transmission of parasites to marine fish. Parasitology, 124, 83-99.

Marcogliese, D. J., and Cone, D. K. (1997). Food webs: a plea for parasites. Trends in Ecology and Evolution, 12, 320-325.

Marczak, L. B., Richardson, J. S., and Claessen, M. C. (2006). Life history phenology and sediment size association of the dragonfly Cordulegaster dorsalis (Odonata: Cordulgastridae) in an ephemeral habitat in southwestern British Columbia. The Canadian Field Naturalist, 120, 347-350.

Marsit, C. J., Fried, B., and Sherma, J. (2000a). Neutral lipids in cercariae, encysted metacercariae, and rediae of Zygocotyle lunata. Journal of Parasitology, 86, 1162-1163.

Marsit, C. J., Fried, B., and Sherma, J. (2000b). Neutral lipids in cercariae, encysted metacercariae, and rediae of Echinostoma caproni. Journal of Helminthology, 74, 365367. 
Martin-Creuzburg, D., Kowarik, C., and Straile, D. (2017). Cross-ecosystem fluxes: Export of polyunsaturated fatty acids from aquatic to terrestrial ecosystems via emerging insects. Science of The Total Environment, 577, 174-182.

Massoud, J. (1974). The effect of variation in miracidial exposure dose on laboratory infections of Ornithobilharzia turkestanicum in Lymnaea gedrosiana. Journal of Helminthology, 48, 139-144.

May, R. M. (1973). Qualitative stability in model ecosystems. Ecology, 54, 638-641.

Mayes, P. A., and Botham, K. M. (2003). Metabolism of unsaturated fatty acids and eicosanoids. Harper's Illustrated Biochemistry, 26th Ed. (Lange Medical Books, New York) p, 190-196.

McCann, K. S. (2000). The diversity-stability debate. Nature, 405, 228-233.

McCarthy, A.M. (1999). The influence of temperature on the survival and infectivity of the cercariae of Echinoparyphium recurvatum (Digenea: Echinostomatidae). Parasitology, $118,383-388$.

McCauley, S. J. (2008). Slow, fast and in between: habitat destruction and behavior of larvae in nine species of libellulid dragonfly. Freshwater Biology, 53, 253-263.

McDonald, J. (2008). Handbook of Biological Statistics. Sparky House Publishing.

McLaren, I. A., Laberge, E., Corkett, C. J., and Sevigny, J. M. (1989). Life cycles of four species of Pseudocalanus in Nova Scotia. Canadian Journal of Zoology, 67, 552-558.

McManus, D. P., Marshall I., James B. L. (1975). Lipids in digestive gland of Littorina saxatilis rudis (Maton) and in daughter sporocysts of Microphallus similis (Jag 1900). Experimental Parasitology, 37, 157-163.

McNamara R. K., and Carlson S. E. (2006). Role of omega-3 fatty acids in brain development and function: potential implications for the pathogenesis and prevention of psychopathology. Prostaglandins, Leukotrienes and Essential Fatty Acids, 75, 329-49.

Menendez, J. A., and Lupu, R. (2007). Fatty acid synthase and the lipogenic phenotype in cancer pathogenesis. Nature Reviews Cancer, 7, 763.

Meyer, F., Meyer, H., and Bueding, E. (1970). Lipid metabolism in the parasitic and free-living flatworms, Schistosoma mansoni and Dugesia dorotocephala. Molecular and Cell Biology of Lipids, 210, 257-266.

Misra, K. K., Shkrob, I., Rakshit, S., and Dembitsky, V. M. (2002). Variability in fatty acids and fatty aldehydes in different organs of two prosobranch gastropod mollusks. Biochemical Systematics and Ecology, 30, 749-761. 
Montoya, J. M., Pimm, S. L., and Solé, R. V. (2006). Ecological networks and their fragility. Nature, 442, 259-264.

Moore, M. N., and Halton, D. W. (1975). A histochemical study of the rediae and cercariae of Fasciola hepatica. Parasitology Research, 47, 45-54.

Morley, N. J. (2012). Cercariae (Platyhelminthes: Trematoda) as neglected components of zooplankton communities in freshwater habitats. Hydrobiologia, 691, 7-19.

Müller-Navarra, D. C. (1995). Evidence that a highly unsaturated fatty acid limits Daphnia growth in nature. Archiv für Hydrbiologie, 132, 297-307.

Müller-Navarra, D. C. (2006). The nutritional importance of polyunsaturated fatty acids and their use as trophic markers for herbivorous zooplankton: Does it contradict? Archiv für Hydrobiologie, 167, 501-513.

Müller-Navarra, D., Brett, M. T., Liston, A. M., Goldman, C. R. (2000). A highly unsaturated fatty acid predicts carbon transfer between primary producers and consumers. Nature, 403, 74-77.

Müller, E. E., Fried D., and Sherma J. (2000) HPTLC analysis of neutral lipids in Biomphalaria glabrata snails infected with Schistosoma mansoni (Trematoda). Journal of Planar Chromatography-Modern TLC, 13, 228-231.

Muller, E. E., Fried, B., and Sherma, J. (1999). HPTLC determination of neutral lipids in the cercariae of Echinostoma trivolvis and Echinoparyphium sp. (Trematoda). Journal of Planar Chromatography, Modern TLC, 12, 306-308.

Nakano, S., and Murakami, M. (2001). Reciprocal subsidies: dynamic interdependence between terrestrial and aquatic food webs. Proceedings of the National Academy of Sciences, 98, 166-170.

Nanduri, J., Dennis, J. E., Rosenberry, T. L., Mahmoud, A. A. F., and Tartakoff, A. M. (1991). Glycocalyx of bodies versus tails of Schistosoma mansoni cercariae (lectin-binding, size, charge, and electron microscopic characterization). Journal of Biological Chemistry, 266, 1341-1347.

Odum, E. P. (1953). Fundamentals of ecology 1971, 3rd edn. (London, Saunders. 1971).

Orlofske S. A., Jadin R. C., Preston D. L., and Johnson P. T. J. (2012) Parasite transmission in complex communities: predators and alternative hosts alter pathogenic infections in amphibians. Ecology, 93, 1247-125.

Orlofske, S. A., Jadin, R. C., and Johnson, P. T. (2015). It's a predator-eat-parasite world: how characteristics of predator, parasite and environment affect consumption. Oecologia, 178, 537-547. 
Oudejans, R. C. H. M. and Van der Horst, D. J. (1974). Effect of excessive fatty acid ingestion of new lipids and phospholipids of snail Helix pornatia L. Lipids, 9, 798-803.

Paine, R. T., (1980). Food webs: linkage, interaction strength and community infrastructure. Journal of Animal Ecology, 49, 667-685.

Parrish, C. C. (1999). Determination of total lipid, lipid classes, and fatty acids in aquatic samples. In Lipids in Freshwater Ecosystems (pp. 4-20). Springer, New York, NY.

Parrish, C. C. (2009). Essential fatty acids in aquatic food webs. In Lipids in Aquatic Ecosystems. Edited by M.T. Arts, M.T. Brett, and M.J. Kainz. Springer. New York, pp. 309-326.

Pascual, M., and Dunne, J. A. (Eds.). (2006). Ecological networks: linking structure to dynamics in food webs. Oxford University Press.

Paull, S. H., and Johnson, P. T. J. (2011). High temperature enhances host pathology in snailtrematode system: possible consequences of climate change for the emergence of disease. Freshwater Biology, 56, 767-778.

Paull, S. H., Raffel, T. R., LaFonte, B. E., Johnson, P. T. J. (2015). How temperature shifts affect parasite production: testing the roles of thermal stress and acclimation. Functional Ecology, 29, 941-950.

Pechenik, J. A. and Fried, B. (1995). Effect of temperature on survival and infectivity of Echinostoma trivolvis cercariae: a test of the energy limitation hypothesis, Parasitology, $111,373-378$.

Pereira, A. S. A., Cavalcanti, N. L., Nascimento, G. A. F., Nascimento-Silva, J. L. G., Padilha, R. J. R., Viegas, L. F. W., Alves, L. C., Lima-Filho, J. L., Chaves, M. E. C. (2013). Morphological and morphometric study of cercariae and adult worms of Schistosoma mansoni (SLM strain) isolated from infected mice. Parasitological Research, 112, 1087-1096.

Persson, J., and Vrede, T. (2006). Polyunsaturated fatty acids in zooplankton: variation due to taxonomy and trophic position. Freshwater Biology, 51, 887-900.

Pfluger, W., 1980. Experimental epidemiology of Schistosomiasis. I. The prepatent period and cercarial production of Schistosoma mansoni in Biomphalaria snails at various constant temperatures. Zeitschrift fur Parasitenkunde, 63, 159-169.

Phang, M., Lazarus, S., Wood, L. G., Garg, M. (2011). Diet and thrombosis risk: nutrients for prevention of thrombotic disease. Seminars in Thrombosis and Hemostasis, 37, 199-208.

Pinheiro, J., Júnior, A. M., and Lanfredi, R. M. (2009). Physiological changes in Lymnaea columella (Say, 1817) (Mollusca, Gastropoda) in response to Echinostoma paraensei (Lie 
and Basch, 1967) (Trematoda: Echinostomatidae) infection. Parasitology Research, 106, $55-59$.

Plourde, M., and Cunnane, S. C. (2007). Extremely limited synthesis of long chain polyunsaturates in adults: implications for their dietary essentiality and use as supplements. Applied Physiology, Nutrition, and Metabolism, 32, 619-34.

Polis, G. A., and Hurd, S. D. (1996). Allochthonous input across habitats, subsidized consumers, and apparent trophic cascades: examples from the ocean-land interface. In Food Webs (pp. 275-285). Springer, Boston, MA.

Pond, C. M. (1981). Storage, p. 190-219. In C. R. Townsend and P. Calow [eds.], Physiological Ecology.

Poulin, R. (1999). The functional importance of parasites in animal communities: many roles at many levels? International Journal for Parasitology, 29, 903-914.

Poulin, R. (2006). Global warming and temperature-mediated increases in cercarial emergence in trematode parasites. Parasitology, 132, 143-151.

Poulin, R. (2011). Uneven distribution of cryptic diversity among higher taxa of parasitic worms. Biology Letters, 7, 241-244.

Preston, D. L., Orlofske, S. A., Lambden, J. P., and Johnson, P. T. (2013). Biomass and productivity of trematode parasites in pond ecosystems. Journal of Animal Ecology, 82, 509-517.

Preston, D., and Johnson, P. (2010). Ecological consequences of parasitism. Nature.

Price, P. W. (1980). Evolutionary Biology of Parasites (Vol. 15). Princeton University Press.

Priscu, J. C., Priscu, L. R., Palmisano, A. C., and Sullivan, C. W. (1990). Estimation of neutral lipid levels in Antarctic sea ice microalgae by Nile red fluorescence. Antarctic Science, 2, $149-155$.

Pritchard, G. (1964). The prey of dragonfly larvae (Odonata; Anisoptera) in ponds in Northern Alberta. Canadian Journal of Zoology, 42, 785-799.

Ravet, J. L., Brett, M. T., and Müller-Navarra, D. C. (2003). A test of the role of polyunsaturated fatty acids in phytoplankton food quality for Daphnia using liposome supplementation. Limnology and Oceanography, 48, 1938-1947.

Ravet, J., Brett, M. T., and Arhonditsis, G. (2010). The effects of seston lipids on zooplankton fatty acid composition in Lake Washington, Washington, USA. Ecology, 91, 180-190. 
Salafsky, B., and Fusco, A. C. (1987). Schistosoma mansoni: a comparison of secreted vs nonsecreted eicosanoids in developing schistosomulae and adults. Experimental Parasitology, 64, 361-367.

Salafsky, B., Wang, Y. S., Fusco, A. C., and Antonacci, J. (1984). The role of essential fatty acids and prostaglandins in cercarial penetration (Schistosoma mansoni). The Journal of Parasitology, 656-660.

Sánchez, M. I., Thomas, F., Perrot-Minnot, M. J., Biron, D. G., Bertrand-Michel, J., and Missé, D. (2009). Neurological and physiological disorders in Artemia harboring manipulative cestodes. Journal of Parasitology, 95, 20-24.

SanGiovanni J. P., and Chew E. Y. (2005). The role of omega-3 long-chain polyunsaturated fatty acids in health and disease of the retina. Progress in Retinal and Eye Research, 24, 87138.

Sanzone, D. M., Meyer, J. L., Marti, E., Gardiner, E. P., Tank, J. L., and Grimm, N. B. (2003). Carbon and nitrogen transfer from a desert stream to riparian predators. Oecologia, 134, 238-250.

Sargent, J. R. and R. J. Henderson. (1986). Lipids. In E. D. S. Corner and S. C. M. O'Hara (eds), The Biological Chemistry of Marine Copepods. Clarendon Press, Oxford: 59-164.

Scaife, J. R., Moyo, J., Galbraith, H., Michie, W., and Campbell, V. (1994). Effect of different dietary supplemental fats and oils on the tissue fatty acid composition and growth of female broilers. British Poultry Science, 35, 107-118.

Schariter, J. A., Pachuski, J., Fried, B., and Sherma, J. (2002). Determination of neutral lipids and phospholipids in the cercariae of Schistosoma mansoni by high performance thin layer chromatography. Journal of Liquid Chromatography and Related Technologies, 25, 1615-1622.

Schell, S. C. (1985). Handbook of trematodes of North America north of Mexico. University Press of Idaho.

Schlechtriem, C., Arts, M. T., \& Zellmer, I. D. (2006). Effect of temperature on the fatty acid composition and temporal trajectories of fatty acids in fasting Daphnia pulex (Crustacea, Cladocera). Lipids, 41, 397-400.

Schmitz, G., and Ecker, J. (2008). The opposing effects of $n-3$ and $n-6$ fatty acids. Progress in Lipid Research, 47, 147-155.

Scholtz, N., Roulin, A., Ebert, D., and Martin-Creuzburg, D. (2016). Combined effects of dietary polyunsaturated fatty acids and parasite exposure on eicosanoid-related gene expression in an invertebrate model. Comparative Biochemistry and Physiology Part A: Molecular and Integrative Physiology, 201, 115-123. 
Schotthoefer, A. M., K. M. Labak, and V. R. Beasley. (2007). Ribeiroia ondatrae cercariae are consumed by aquatic invertebrate predators. Parasitology, 93, 1240-1243.

Schotthoefer, A. M., Labak, K. M., and Beasley, V. R. (2007). Ribeiroia ondatrae cercariae are consumed by aquatic invertebrate predators. Journal of Parasitology, 93, 1240-1243.

Shetty, P. H., Fried, B., and Sherma, J. (1992). Effects of patent Echinostoma caproni infection on the sterol composition of the digestive gland-gonad complex of Biomphalaria glabrata as determined by gas-liquid chromatography. Journal of Helminthology, 66, 6871.

Shul'man, G. E., and Yuneva, T. V. (1990). Docosahexaenoic acid and unsaturation of lipids in fish (In Russian). Gydmbiologicheskii Zhurnal, 26, 50-55.

Sikora, A. B., Petzoldt, T., Dawidowicz, P., and Von Elert, E. (2016). Demands of eicosapentaenoic acid (EPA) in Daphnia: are they dependent on body size? Oecologia, $182,405-417$.

Simopoulos, A. P. (1999). Essential fatty acids in health and chronic disease. The American Journal of Clinical Nutrition, 70, 560-569.

Simopoulos, A. P. (2011). Evolutionary Aspects of Diet: The Omega-6/Omega-3 Ratio and the Brain. Molecular Neurobiology, 44, 203-215.

Sinensky, M. (1974). Homeoviscous adaptation-a homeostatic process that regulates the viscosity of membrane lipids in Escherichia coli. Proceedings of the National Academy of Sciences, 71, 522-525.

Smith, T. S., Brooks, T. J., and White, H. B. (1966). Thin-layer and gas-liquid chromatographic analysis of lipid from cercariae of Schistosoma mansoni. American Journal of Tropical Medicine and Hygiene, 15, 307-313.

Smyntek, P. M., Teece, M. A., Schulz, K. L., and Storch, A. J. (2008). Taxonomic differences in the essential fatty acid composition of groups of freshwater zooplankton relate to reproductive demands and generation time. Lipids, 46, 709-721.

Soldánová, M., Selbach, C., and Sures, B. (2016). The early worm catches the bird? Productivity and patterns of Trichobilharzia szidati cercarial emission from lymnaea stagnalis. PloS One, 11, e0149678.

Soldánová, M., Selbach, C., and Sures, B. (2016). The early worm catches the bird? Productivity and patterns of Trichobilharzia szidati cercarial emission from Lymnaea stagnalis. PloS One, 11, e0149678. 
Sorensen, R. E., and Minchella, D. J. (1998). Parasite influences on host life history: Echinostoma revolutum parasitism of Lymnaea elodes snails. Oecologia, 115, 188-195.

Sousa, W. P. (1983). Host life history and the effect of parasitic castration on growth: a field study of Cerithidea californica Haldeman (Gastropoda: Prosobranchia) and its trematode parasites. Journal of Experimental Marine Biology and Ecology, 73, 273-296.

Sousa, W. P. (1983). Host life history and the effect of parasitic castration on growth: A field study of Cerithidea californica Haldeman (Gastropoda: Prosobranchia) and its trematode parasites. Journal of Experimental Marine Biology and Ecology, 73, 273-296.

Southgate, V. R. (1970). Observations on the epidermis of the miracidium and on the formation of the tegument of the sporocyst of Fasciola hepatica. Parasitology, 61, 177-190.

Stanley, D. W., and Miller, J. S. (2006). Eicosanoid actions in insect cellular immune functions. Entomologia Experimentalis et Applicata, 119, 1-13.

Stanley, D., and Kim, Y. (2014). Eicosanoid signaling in insects: from discovery to plant protection. Critical Reviews in Plant Sciences, 33, 20-63.

Stillwell, W. and Wassall, S. R. (2003). Docosahexaenoic acid: membrane properties of a unique fatty acid. Chemistry and Physics of Lipids, 126, 1-27.

Stoddart, J. A. (1982). Ingestion of cercariae by a bryozoan. Journal of Parasitology, 68, 1137.

Storms, Z. J., Cameron, E., de la Hoz Siegler, H., and McCaffrey, W. C. (2014). A simple and rapid protocol for measuring neutral lipids in algal cells using fluorescence. Journal of visualized experiments: JoVE, 87, doi: 10.3791/51441.

Strandberg, U., Hiltunen, M., Jelkänen, E., Taipale, S. J., Kainz, M. J., Brett, M. T., and Kankaala, P. (2015). Selective transfer of polyunsaturated fatty acids from phytoplankton to planktivorous fish in large boreal lakes. Science of the Total Environment, 536, 858865.

Sukhdeo, M. V. (2012). Where are the parasites in food webs?. Parasites and Vectors, 5, 239.

Sukhdeo, M., and Sukhdeo, S. (2004). Trematode behaviours and the perceptual worlds of parasites. Canadian Journal of Zoology, 82, 292-315.

Sushchik, N. N., Gladyshev, M. I., Makhutova, O. N., Kalachova, G. S., Kravchuk, E. S., and Ivanova, E. A. (2004). Associating particulate essential fatty acids of the 13 family with phytoplankton species composition in a Siberian reservoir. Freshwater Biology, 49, 1206-1219.

Szuroczki, D. and Richardson, J. M. (2009). The role of trematode parasites in larval anuran communities: an aquatic ecologist's guide to the major players. Oecologia, 161, 371-385. 
Taipale, S., Strandberg, U., Peltomaa, E., Galloway, A. W. E., Ojala, A. and Brett, M. (2013). Fatty acid composition as biomarkers of freshwater microalgae: analysis of 37 strains of microalgae in 22 genera and in seven classes. Aquatic Microbial Ecology, 71, 165-178.

Tessier, A. J., and Goulden, C. E. (1982). Estimating food limitation in cladoceran populations. Limnology and Oceanography, 27, 707-717.

Tessier, A. J., Henry, L. L., Goulden, C. E., and Durand, M. W. (1983). Starvation in Daphnia: energy reserves and reproductive allocation. Limnology and Oceanography, 28, 667-676.

Théron, A., Moné, H., and Gérard, C. (1992). Spatial and energy compromise between host and parasite: the Biomphalaria glabrata-Schistosoma mansoni system. International Journal for Parasitology, 22, 91-94.

Thieltges, D. W., Amundsen, P. A., Hechinger, R. F., Johnson, P. T., Lafferty, K. D., Mouritsen, K. N., Preston, D. L., Reise, K., Zander, C. D., and Poulin, R. (2013). Parasites as prey in aquatic food webs: implications for predator infection and parasite transmission. Oikos, 122, 1473-1482.

Thieltges, D. W., de Montaudouin, X., Fredensborg, B., Jensen, K. T., Koprivnikar, J., and Poulin, R. (2008). Production of marine trematode cercariae: a potentially overlooked path of energy flow in benthic systems. Marine Ecology Progress Series, 372, 147-155.

Thieltges, D. W., de Montaudouin, X., Fredensborg, B., Jensen, K. T., Koprivnikar, J., and Poulin, R. (2008). Production of marine trematode cercariae: a potentially overlooked path of energy flow in benthic systems. Marine Ecology Progress Series, 372, 147-155.

Thompson, R. M., Brose, U., Dunne, J. A., Hall, R. O., Hladyz, S., Kitching, R. L., Martinez, N. D., Rantala, O. H., Romanuk, T. N., Stouffer, D. B. and Tylianakis, J. M. (2012). Food webs: reconciling the structure and function of biodiversity. Trends in Ecology and Evolution, 27, 689-697.

Thompson, R. M., Mouritsen, K. N., and Poulin, R. (2005). Importance of parasites and their life cycle characteristics in determining the structure of a large marine food web. Journal of Animal Ecology, 74, 77-85.

Thompson, S. N. (1987). Effect of Schistosoma mansoni on the gross lipid composition of its vector Biomphalaria glabrata. Comparative Biochemistry and Physiology Part B: Comparative Biochemistry, 87, 357-360.

Thompson, S. N., Mejia-Scales, V., and Borchardt, D. B. (1991). Physiologic studies of snailschistosome interactions and potential for improvement of in vitro culture of schistosomes. In Vitro Cellular and Developmental Biology-Animal, 27, 497-504. 
Tkach, V., Pawlowski, J., Mariaux, J. (2000). Phylogenetic analysis of the suborder Plagiorchiata (Platyhelminthes, Digenea) based on partial lsrDNA sequences1. International Journal for Parasitology, 30, 83-93.

Tokobaev, M. M., Kutikova, L. A. and Chibichenko, N. T. (1979). The predatory rotifer, Eosphora ehrenbergi, a biological eliminator of cercariae in Lake Issyk-Kul'. Trudy Gel'mintologicheskoi Laboratorii Gel'minty zhivotnykh i rastenii, 29, 146-149. [In Russian].

Tokobaev, M. M., L. A. Kutikova and N. T. Chibichenko. (1979). The predatory rotifer, Eosphora ehrenbergi, a biological eliminator of cercariae in Lake Issyk-Kul'. Trudy Gel'mintologicheskoi Laboratorii Gel'minty zhivotnykh i rastenii, 29, 146-149. [In Russian].

Tolstenkov, O. O., Akimova, L. N., Terenina, N. B., Gustafsson, M. K. S. (2012b). The neuromuscular system in continuously swimming cercariae from Belarus. I Xiphidiocercariae.

Tunholi-Alves, V. M., Tunholi, V. M., Gôlo, P., Lustrino, D., Maldonado Jr, A., Bittencourt, V. R. E. P., Rodrigues M. L. A., and Pinheiro, J. (2011). Lipid levels in Biomphalaria glabrata infected with different doses of Echinostoma paraensei miracidia. Experimental Parasitology, 128, 212-216.

Twining, C. W., Brenna, J. T., Lawrence, P., Shipley, J. R., Tollefson, T. N., and Winkler, D. W. (2016). Omega-3 long-chain polyunsaturated fatty acids support aerial insectivore performance more than food quantity. Proceedings of the National Academy of Sciences, 113, 10920-10925.

Vadeboncoeur, Y., McIntyre, P. B., and Vander Zanden, M. J. (2011). Borders of biodiversity: life at the edge of the world's large lakes. BioScience, 61, 526-537.

Valles-Regino, R., Tate, R., Kelaher, B., Savins, D., Dowell, A., and Benkendorff, K. (2015). Ocean warming, and CO2-induced acidification impact the lipid content of a marine predatory gastropod. Marine Drugs, 13, 6019-6037.

Van der Horst, D. J. (1973). Biosynthesis of saturated and unsaturated fatty acids in the pulmonate land snail Cepaea nemoralis (L.). Comparative Biochemistry and Physiology, 46, 551-560.

Van Regenmortel, M. H. (2003). Viruses are real, virus species are man-made, taxonomic constructions. Archives of virology, 148(12), 2481-2488.

Veloza, A. J., Chu, F. L. E, and Tang, K. W. (2006). Trophic modification of essential fatty acids by heterotrophic protists and its effects on the fatty acid composition of the copepod Acartia tonsa. Marine Biology, 148, 779-788. 
Von Brand, T. (1973). Biochemistry of Parasites, 2nd Ed. Academic Press, New York, p. 499.

Von Elert, E. (2002). Determination of limiting polyunsaturated fatty acids in Daphnia galeata using a new method to enrich food algae with single fatty acids. Limnology Oceanographer, 47, 1764-1773.

Waldock, M. J., and Holland, D. L. (1984). Fatty acid metabolism in young oysters, Crassostrea gigas: polyunsaturated fatty acids. Lipids, 19, 332-336.

Walker, E. M., and P. S. Corbet. (1975). The Odonata of Canada and Alaska. University of Toronto Press. Volume 3, Part III: The Anisoptera - Three Families, 257.

Wall R., Ross R. P., Fitzgerald G. F., and Stanton C. (2010). Fatty acids from fish: the antiinflammatory potential of long-chain omega-3 fatty acids. Nutrition Reviews, 68, 280289.

Wang Y., Lin D. S., Bolewicz L., Connor W. E. (2006). The predominance of polyunsaturated fatty acids in the butterfly Morpho peleides before and after metamorphosis. Journal of Lipid Research, 47, 530-536.

Warren, C. P., Pascual, M., Lafferty, K. D., and Kuris, A. M. (2010). The inverse niche model for food webs with parasites. Theoretical Ecology, 3, 285-294.

Weers, P., Siewertsen, K., and Gulati, R. (1997). Is the fatty acid composition of Daphnia galeata determined by the fatty acid composition of the ingested diet? Freshwater Biology, 48, 731-738.

Welsh, J. E., Liddell, C., Van Der Meer, J., and Thieltges, D. W. (2017). Parasites as prey: the effect of cercarial density and alternative prey on consumption of cercariae by four nonhost species. Parasitology, 144, 1775-1782.

White, M., Fried, B., and Sherma, J. (2006). Determination of the effects of estivation and starvation on neutral lipids in phospholipids in Biomphalaria glabrata (NMRI strain) and Helisoma trivolvis (Colorado strain) snails by quantitative high performance thin layer chromatography-densitometry. Journal of Liquid Chromatography and Related Technologies, 29, 2167-2180.

Widdows, J., and Bayne, B. L. (1971). Temperature acclimation of Mytilus edulis with reference to its energy budget. Journal of the Marine Biological Association of the United Kingdom, 51(4), 827-843.

Williams, R. J., and Martinez, N. D. (2000). Simple rules yield complex food webs. Nature, 404, 180-183. 
Winemiller, K. O. and Polis, G. A. (1996). Food webs: what can they tell us about the world? In: Food webs: integration of patterns and dynamics (ed. G. A. Polis GA and K. O. Winemiller). New York, Chapman and Hall.

Wissinger, S. A. (1989). Seasonal variation in the intensity of competition and predation among dragonfly larvae. Ecology, 70, 1017-1027.

Wood, C. L., and Johnson, P. T. (2015). A world without parasites: exploring the hidden ecology of infection. Frontiers in Ecology and the Environment, 13, 425-434.

Xi, Y. L., Liu, G. Y., and Jin, H. J. (2002). Population growth, body size, and egg size of two different strains of Brachionus calyciflorus Pallas (Rotifera) fed different algae. Journal of Freshwater Ecology, 17, 185-190.

Xu, X. F., Stack, R. J., Rao, N., and Caulfield, J. P. (1994). Schistosoma mansoni: fractionation and characterization of the glycocalyx and glycogen-like material from cercariae. Experimental Parasitology, 79, 399-409.

Zd'árská, Z. (1968). The histology and histochemistry of the cystogenic cells of the cercaria Echinoparyphium aconiatum, Dietz, 1909. Folia Parasitologica, 15, 213-232.

Zd'árská, Z. (1970). The gland cells of the cercaria of Notocotylus attenuatus (Rudolphi, 1809) and the cyst wall of its adolescaria. Folia Parasitologica, 17, 31-47.

Zd'árská, Z. (1971). The cystogenic gland cells of the cercaria of Philophthalmus sp. Folia Parasitologica, 18, 119-125.

Zd'árská, Z. (1979). Histochemistry of gland cells of Eurytrema pancreaticum cercariae. Folia Parasitologica, 26, 259-263.

Zimmer, R., Fingernut, J., and Zimmer, C. (2009). Dispersal pathways, seed rains, and the dynamics of larval behaviour. Ecology, 90, 1933-1947. 